Beiträge aus der Nordwestdeutschen Forstlichen Versuchsanstalt Band 7

\title{
Das digitale Luftbild
}

Ein Praxisleitfaden für Anwender im Forst- und Umweltbereich Arbeitsgruppe Forstlicher Luftbildinterpreten
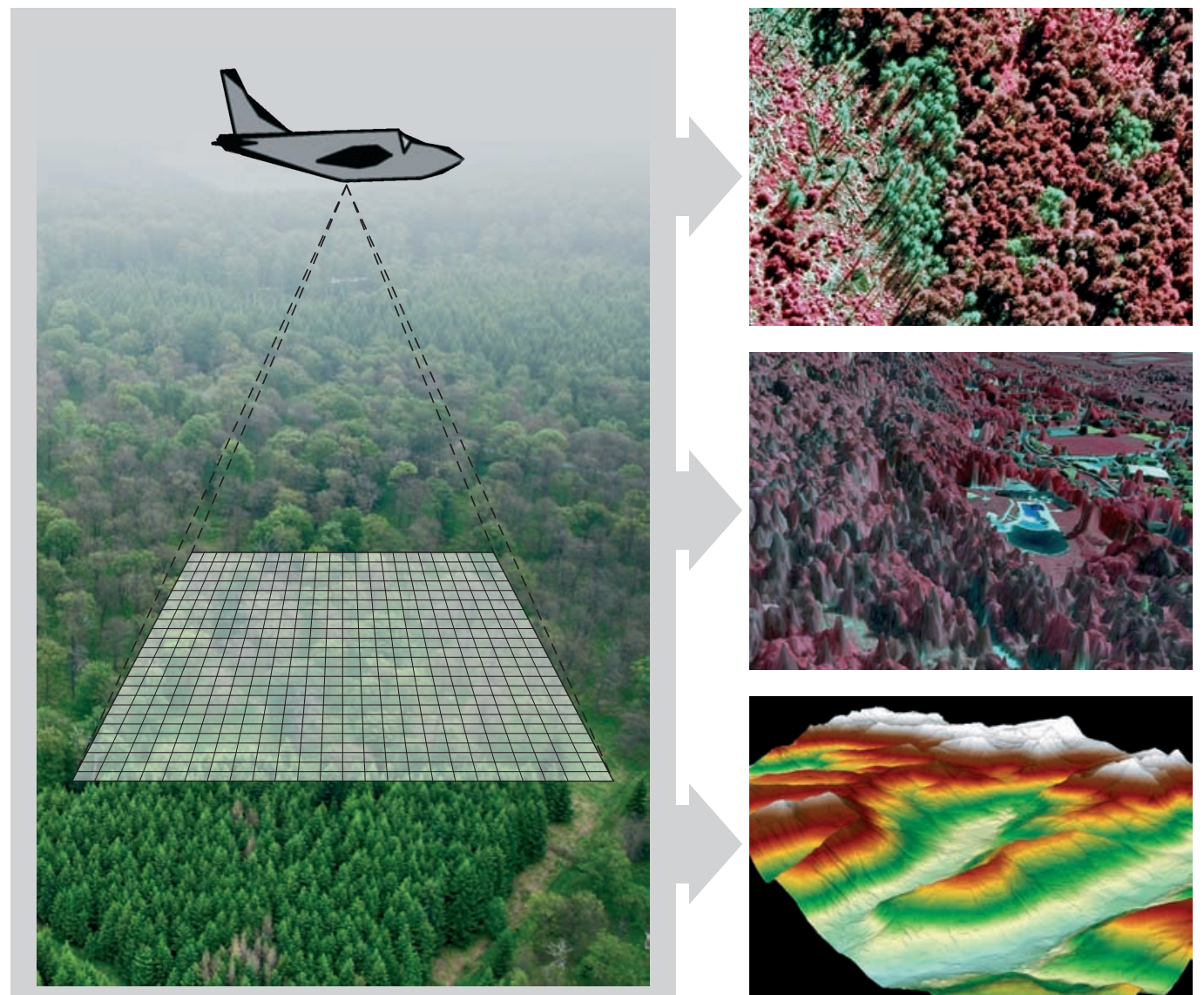

G⿻弋一⿻上丨 Universitätsdrucke Göttingen
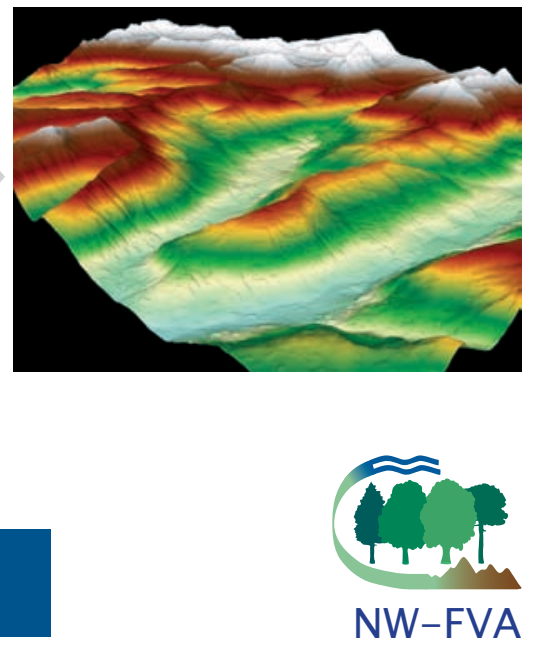

Arbeitsgruppe Forstlicher Luftbildinterpreten (Hrsg.)

Das digitale Luftbild

This work is licensed under the Creative Commons License 3.0 "by-nd", allowing you to download, distribute and print the document in a few copies for private or educational use, given that the document stays unchanged and the creator is mentioned.

You are not allowed to sell copies of the free version.

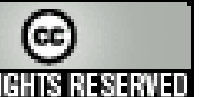

SORE RIIGHIS RESERVED 
erschienen als Band 7 der Reihe „Beiträge aus der Nordwestdeutschen Forstlichen Versuchsanstalt" in den Universitätsdrucken im Universitätsverlag Göttingen 2012 
Arbeitsgruppe Forstlicher

Luftbildinterpreten (Hrsg.)

\section{Das digitale Luftbild}

Ein Praxisleitfaden für Anwender im

Forst- und Umweltbereich

Beiträge aus der

Nordwestdeutschen

Forstlichen Versuchsanstalt

Band 7

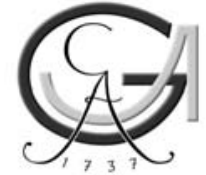

Universitätsverlag Göttingen 2012 


\section{Bibliographische Information der Deutschen Nationalbibliothek}

Die Deutsche Nationalbibliothek verzeichnet diese Publikation in der Deutschen Nationalbibliographie; detaillierte bibliographische Daten sind im Internet über $<$ http://dnb.ddb.de> abrufbar.

Global Forest Decimal Classification: 585, 587, 587.1, 587.2, 587.3

\section{Herausgeber der Reibe:}

Nordwestdeutsche Forstliche Versuchsanstalt (NW-FVA)

Grätzelstr. 2, D-37079 Göttingen

Tel.: +49 (0)551-69401-0, Fax: +49 (0)551-69401-160

E-Mail: zentrale@nw-fva.de

www.nw-fva.de

Schriftleitung der Reibe: Prof. Dr. Hermann Spellmann

Redaktion der Reibe: Inge Kehr, Ulrike Gaertner

Redaktion: Jörg Ackermann

Autoren: Jörg Ackermann, Dr. Petra Adler, Christoph Bauerhansl, Uwe Brockamp, Friedrich Engels, Frank Franken, Christian Ginzler, Dr. Claus-Peter Gross, Karina Hoffmann, Kai Jütte, Prof. Dr. Hartmut Kenneweg, Dr. Tatjana Koukal, Dr. Klaus Martin, Bruno Regner, Rudolf Seitz, Armin Troycke

\section{Titelgrafik: Etta Paar (NW-FVA)}

Dieses Buch ist auch als freie Onlineversion über die Homepage der NW-FVA, des Verlages sowie über den OPAC der Niedersächsischen Staats- und Universitätsbibliothek (http://www.sub.uni-goettingen.de) erreichbar und darf gelesen, heruntergeladen sowie als Privatkopie ausgedruckt werden. Es gelten die Lizenzbestimmungen der Onlineversion. Es ist nicht gestattet, Kopien oder gedruckte Fassungen der freien Onlineversion zu veräußern.

(C) 2012 Universitätsverlag Göttingen

http://univerlag.uni-goettingen.de

ISBN: 978-3-86395-055-2

ISSN: $1865-6994$ 


\section{Inhaltsverzeichnis}

Abbildungsverzeichnis IV

Tabellenverzeichnis $\quad$ V

Zusammenfassung 1

$\begin{array}{ll}\text { Abstract } & 2\end{array}$

1 Einführung 3

2 Digitale Luftbildkameras 5

2.1 Zeilenkameras 5

2.2 Matrixkameras 7

2.2.1 Großformatkameras 9

2.2.2 Mittelformatkameras 12

$\begin{array}{ll}2.2 .3 & \text { Kleinformatkameras } \\ \end{array}$

2.3 Technische Parameter 12

2.3.1 Spektrale Auflösung 12

$\begin{array}{ll}\text { 2.3.2 Radiometrische Auflösung } & 14\end{array}$

$\begin{array}{ll}\text { 2.3.3 Bodenauflösung und Abdeckung } & 15\end{array}$

2.3.4 Dateigrößen 16

3 Digitalisierung analoger Luftbilder 19

$\begin{array}{ll}3.1 \text { Scannertypen } & 19\end{array}$

3.2 Bildauflösung und Dateigrößen 20

3.2.1 Scanauflösung $\quad 21$

3.2.2 Radiometrische Auflösung 22

3.2.3 Dateigrößen 22

3.3 Bildbearbeitung beim Scannen 24

3.4 Kosten für Luftbildscans $\quad 24$

4 Planung, Vergabe und Durchführung digitaler Bildflüge 25

4.1 Technische Rahmenbedingungen 25

4.2 Äußere Rahmenbedingungen 26

4.3 Flugplanung 26

4.4 Datenübergabe 28

4.5 Kosten einer Luftbildbefliegung 29 
5 Bildorientierung 31

5.1 Innere Orientierung 31

5.2 Äußere Orientierung 31

5.3 Aerotriangulation 32

6 Orthophotoerstellung 33

7 Auswertung digitaler Luftbilder $\quad 41$

7.1 Die visuelle Auswertung digitaler Orthophotos 41

7.2 Die visuelle Auswertung digitaler Stereoluftbilder 43

7.3 Die teilautomatisierte Auswertung digitaler Luftbilder 45

7.4 Aktuelle praktische Beispiele 48

8 Datenmanagement $\quad 57$

8.1 Konzepte der Datenhaltung $\quad 57$

8.1.1 Zentrale, filebasierte Datenhaltung 57

8.1.2 Zentrale, datenbankbasierte Datenhaltung 58

8.1.3 Dezentrale, filebasierte Datenhaltung 58

8.1.4 Dezentrale, datenbankbasierte Datenhaltung 59

8.1.5 Rasterdatenmanagementsysteme 59

8.2 Hardwareausstattung von Datenservern $\quad 60$

8.3 Softwareausstattung eines Datenbankservers $\quad 62$

8.3.1 Betriebssystem $\quad 62$

8.3.2 Datenbank $\quad 62$

$\begin{array}{lll}\text { 8.3.3 Metadaten } & 62\end{array}$

8.4 Datennetzwerke 63

8.5 Datensicherung 63

9 Hard- und Software für die Verarbeitung digitaler Luftbilder 65

9.1 Hardware 65

9.2 Software 66

$\begin{array}{lll}10 & \text { Fazit } & 67\end{array}$

10.1 Digitale Luftbildkameras (Kapitel 2) 67

10.2 Digitalisierung analoger Luftbilder (Kapitel 3) 67

10.3 Planung, Vergabe und Durchführung digitaler Bildflüge (Kapitel 4) 67

10.4 Bildorientierung (Kapitel 5) 68

10.5 Orthophotoerstellung (Kapitel 6) 68

10.6 Auswertung digitaler Luftbilder (Kapitel 7) 68 
10.7 Datenmanagement (Kapitel 8) 68

10.8 Hard- und Software für die Verarbeitung digitaler Luftbilder (Kapitel 9) 69

$\begin{array}{ll}\text { Literatur } & 71\end{array}$

$\begin{array}{ll}\text { Abkürzungen } & 73\end{array}$

$\begin{array}{ll}\text { Glossar } & 75\end{array}$

$\begin{array}{ll}\text { Autoren } & 81\end{array}$

$\begin{array}{lr}\text { Anhang } & 83\end{array}$

Beiträge aus der NW-FVA, Band 7, 2012 


\section{Abbildungsverzeichnis}

Abbildung 1: Zeilenweise Erfassung der Erdoberfläche mit einer Zeilenkamera 5

Abbildung 2: $\quad$ Korrektur der Winkelwerte bei einem Zeilenkamerabild 6

Abbildung 3: $\quad$ Flächige Erfassung der Erdoberfläche mit einer Matrixkamera 8

Abbildung 4: $\quad$ Ausschnitt aus dem Kalibrierungsprotokoll einer UltraCamXp 8

Abbildung 5: $\quad$ Histogramme unterschiedlich belichteter Luftbilder 14

Abbildung 6: $\quad$ Parameter der äußeren Orientierung 25

Abbildung 7: $\quad$ Längsüberdeckung zwischen Bildern eines Flugstreifens und Querüberdeckung zwischen benachbarten Flugstreifen 27

Abbildung 8: $\quad$ Flughöhenabweichung 27

Abbildung 9: Auswirkung der Orthorektifizierung 33

Abbildung 10: Ablaufschema der Orthophotoerstellung 34

Abbildung 11: Orthophotos, erstellt mit einem Geländemodell aus benachbarten Luftbildern (mit Objektverschiebung) 35

Abbildung 12: $\quad$ Orthophotos, erstellt mit einem Oberflächenmodell aus benachbarten Luftbildern (ohne Objektverschiebung) 36

Abbildung 13: $\quad$ Artefaktbildung bei Verwendung eines DOM 37

Abbildung 14: Kronendarstellung im Stereo-Luftbild und im DGM-basierten Orthophoto 42

Abbildung 15: $\quad$ Lagefehler durch ungenaues DGM 43

Abbildung 16: Bildhomogenisierung durch Beleuchtungskorrektur 46

Abbildung 17: $\quad$ Objektbasierte Klassifikation eines Orthophotos 47

Abbildung 18: $\quad$ Arten der digitalen Datenhaltung 57 


\section{Tabellenverzeichnis}

Tabelle 1: $\quad$ Technische Parameter aktueller Zeilenkameras der Firma Leica Geosystems

Tabelle 2: $\quad$ Technische Parameter aktueller Großformatkameras der Firma Intergraph Z/I Imaging

Tabelle 3: $\quad$ Technische Parameter aktueller Großformatkameras der Firma Microsoft/Vexcel Imaging $\quad 11$

Tabelle 4: $\quad$ Technische Parameter aktueller Mittelformatkameras

Tabelle 5: Bodenauflösung und Bildabdeckung verschiedener Luftbildkameras bei einer Flughöhe von 2.000 m über Grund

Tabelle 6: $\quad$ Dateigrößen unkomprimierter Rohdaten gebräuchlicher Matrixkameras

Tabelle 7: $\quad$ Richtwerte zur Wahl der Scanauflösung in Abhängigkeit vom Photomaterial

Tabelle 8: Bodenauflösung in Abhängigkeit von Bildmaßstab und Scanauflösung

Tabelle 9: $\quad$ Dateigröße eines gescannten Luftbildes in Abhängigkeit von der Scanauflösung

Tabelle 10: Dateigröße je Quadratkilometer abgebildeter Fläche in Abhängigkeit von der Bodenauflösung

Tabelle 11: $\quad$ Herkunft und Eigenschaften digitaler Höhenmodelle 38

Tabelle 12: $\quad$ Vor- und Nachteile einer zentralen, filebasierten Datenhaltung 58

Tabelle 13: $\quad$ Vor- und Nachteile einer zentralen, datenbankbasierten Datenhaltung

Tabelle 14: $\quad$ Vor- und Nachteile einer dezentralen, filebasierten Datenhaltung

Tabelle 15: $\quad$ Vor- und Nachteile einer dezentralen, datenbankbasierten Datenhaltung

Tabelle 16: $\quad$ Vorteile und Funktionalitäten eines Rasterdatenmanagementsystems 



\title{
Das digitale Luftbild \\ - Ein Praxisleitfaden für Anwender im Forst- und Umweltbereich -
}

\author{
Digital aerial images \\ - A manual for users in forestry and environmental \\ disciplines -
}

\section{Zusammenfassung}

Forstwirtschaft, Natur- und Umweltschutz sind Bereiche, in denen traditionell Luftbilder und Geoinformationssysteme intensiv genutzt werden. Die rasante Entwicklung der digitalen Fernerkundung in den letzten Jahren bietet neue Chancen, beinhaltet aber auch große Herausforderungen.

Der vollständige Ersatz von analogen durch digitale Luftbildbefliegungen hat starke Veränderungen bei deren Planung und Durchführung nach sich gezogen. Auch das Management und vor allem die Auswertung digitaler Luftbilddaten verlangen eine gänzlich andere Verfahrensweise als bei den bisherigen analogen Befliegungen.

Die vorliegende Veröffentlichung soll Anwendern im Forst- und Umweltbereich als praxisbezogener Leitfaden für die Arbeit mit digitalen Luftbildern dienen. Sie liefert einen Überblick über die digitale Aufnahmetechnik, die Planung und Durchführung digitaler Luftbildbefliegungen, die Luftbildorientierung, die Orthophotoberechnung, die Auswertung digitaler Luftbilder sowie über das Datenmanagement. Ferner beinhaltet sie detaillierte Hinweise zur Leistungsbe- 
schreibung bei der Ausschreibung und Auftragsvergabe von Luftbildbefliegungen. Ergänzend wird auf die Digitalisierung analoger Luftbilder als Vorstufe für die digitale Bildauswertung eingegangen. Auf technologische Aspekte zu Hard- und Software, die raschen Veränderungen unterliegen, wird nur grundlegend eingegangen.

Stichworte: digitales Luftbild, digitalisiertes Luftbild, digitale Luftbildkameras, Bildflugplanung, forstliche Luftbildauswertung

\begin{abstract}
Forestry, nature conservation and environmental planning are areas in which airborne remote sensing and geographic information systems are traditionally intensively used. The rapid development of digital remote sensing technology in recent years offers new opportunities, but also raises major challenges.

The complete replacement of analogue aerial photography surveys by digital technology has consequently resulted in major changes for planning and implementation of aerial surveys. Both management and, most importantly, the analysis of digital aerial images require completely different approaches from the analogue aerial surveys conducted so far.

This publication provides practical guidance for those working with digital aerial imagery in the fields of forestry and environmental management. It provides an overview of the digital imaging technology, the planning and implementation of digital aerial surveys, orientation of aerial images, orthophoto calculations, analysis and interpretation of digital images as well as data management. Furthermore, it contains detailed guidance for terms of reference for the announcement and awarding of aerial survey contracts. In addition, the digitization of analogue aerial photography, as a preliminary step towards digital image analysis, is also described. Only a fundamental overview of available hard- and software technology is provided due to the rapidity of change in this technology.
\end{abstract}

Keywords: digital aerial images, digitalized aerial photography, digital aerial cameras, planning of image flights, aerial image interpretation in forestry 


\section{Einführung}

Fernerkundungsdaten werden in Mitteleuropa seit Jahrzehnten beim Umweltmonitoring und für die Steuerung forstbetrieblicher Prozesse genutzt. Vor allem flugzeuggestützte, photographische Aufnahmesysteme haben sich hierbei bewährt. Innerhalb der letzten Jahre gab es tiefgreifende technische Veränderungen. Die bis dahin üblichen Luftbildkameras, mit denen großformatige analoge Schwarzweiß-, Farb- oder Farbinfrarotbilder angefertigt wurden, sind innerhalb sehr kurzer Zeit vollständig durch digitale Aufnahmesysteme ersetzt worden. Gleichzeitig sind Programme für die digitale Bildverarbeitung und -auswertung leistungsfähiger und kostengünstiger geworden. Dies ermöglicht nunmehr einem breiteren Anwenderkreis die Nutzung der Digitaltechnik.

Digitale Luftbilder bieten in vielerlei Hinsicht Vorteile. Zunächst liefern sie im Vergleich zu analogen Aufnahmen mehr Informationen. Zudem können bei der digitalen Bildaufbereitung attraktive Produkte wie Orthophotos oder digitale Höhenmodelle mit geringem Zusatzaufwand hergestellt werden. Besonders Orthophotos lassen sich sehr vorteilhaft in kartographischen Projekten einsetzen. Digitale Bilddaten können weiterhin leicht kopiert und schnell anderen Nutzern zur Verfügung gestellt werden. Schließlich bietet die Nutzung digitaler Luftbilder Rationalisierungsmöglichkeiten. Hierdurch lässt sich eine wesentliche Absenkung des Zeit- und Kostenaufwandes in Fernerkundungsprojekten erreichen.

Gleichzeitig stellt die digitale Technik hohe Anforderungen an die Planung von Befliegungen, das Datenmanagement und die Datenauswertung. Während bei analogen Befliegungen durch die Wahl von Filmart, Bildmaßstab und Brennweite der verwendeten Kamera die wesentlichen technischen Parameter definiert werden konnten, erfordert der Einsatz digitaler Aufnahmetechnik mehr Vorüberlegungen. Darüber hinaus fallen bei digitalen Befliegungen erhebliche Datenmengen an. Dies führt zu einem großen Aufwand bei Datenspeicherung, -handhabung und -sicherung. Schließlich verlangt die digitale Bildauswertung ein hoch entwickeltes Fachwissen.

Bisher gibt es noch keine Standards für die Ausschreibung und die Durchführung digitaler Luftbildbefliegungen im Forstbereich. Verschiedene Kamerasysteme mit entsprechend unterschiedlichen Strukturen und Eigenschaften der erzeugten digitalen Daten sind auf dem Markt. Zudem gibt es anwenderseitig erst vergleichsweise wenig praktische Erfahrungen im Hinblick auf die Auswertung digitaler Luftbilder.

Die Arbeitsgruppe Forstlicher Luftbildinterpreten (AFL) hat deshalb den vorliegenden Leitfaden erarbeitet. Ziel des Leitfadens ist es, Anwendern vor allem aus dem forstlichen Umfeld einen Überblick über die Aufnahme und Weiterverarbeitung digitaler Luftbilder zu liefern. Dies soll eine Entscheidungshilfe bei der 
Planung digitaler Befliegungen sowie für den Einstieg in die digitale Bildverarbeitung und -auswertung sein. Zusätzlich beinhaltet der Leitfaden noch Informationen über die Digitalisierung analoger Luftbilder. Auch in Zeiten digitaler Luftbildbefliegungen bleibt dieses Thema aktuell, da umfangreiche Archivbestände analoger Bilder existieren, die wertvolle Informationen für die forstliche Forschung wie auch für forstbetriebliche Fragestellungen liefern.

Allgemeingültige Handlungsempfehlungen können allerdings nicht gegeben werden, da bei der Konzeption digitaler Prozessketten eine Vielzahl von Rahmenbedingungen zu berücksichtigen ist und dies maßgeschneiderte, dem jeweiligen Arbeitsumfeld angepasste Lösungen erfordert. Zudem unterliegen auf dem Markt vorhandene Softwaresysteme für die Verarbeitung und Auswertung digitaler Luftbilder laufenden Veränderungen und Erweiterungen. Deshalb finden sich technische Empfehlungen im Leitfaden nur, soweit sie grundlegender Natur sind und von ihrem Fortbestand ausgegangen werden kann.

Nicht im Leitfaden enthalten ist eine ökonomische Bewertung der Arbeit mit digitalen Luftbildern, da dies nur im Einzelfall möglich ist und zudem eine zeitlich sehr begrenzte Gültigkeit besitzt. 


\section{Digitale Luftbildkameras}

Digitale und analoge Luftbildkameras unterscheiden sich grundlegend. Bei analogen Luftbildkameras wird die reflektierte Strahlung (Reflexion) der aufzunehmenden Objekte auf lichtempfindliche Trägermaterialien (Filme) geleitet. Durch chemische Entwicklungsprozesse entstehen als Endprodukt Negative oder Diapositive.

Bei digitalen Systemen wird die Reflexion über einen elektrooptischen Wandler (CCD-Sensor bzw. -Chip) in digitale Information überführt und gespeichert. Dabei wird durch optische Systeme die Reflexion in Spektralbereiche (Farbkanäle) getrennt und einzelnen CCD-Sensoren zugeführt. Durch Kombination unterschiedlicher Farbkanäle können hieraus Mehrkanal-Bilddaten erzeugt werden.

Digitale Luftbildkameras unterscheiden sich weiterhin von analogen Systemen durch schärfer voneinander abgegrenzte Farbkanäle sowie durch ihre hohe Lichtempfindlichkeit. Dies ermöglicht Aufnahmen auch bei schlechten Beleuchtungsbedingungen, z. B. unter geschlossener Wolkendecke oder bei Winterbefliegungen mit niedrigem Sonnenstand.

Digitale Luftbildkamerasysteme können aufgrund der Anordnung ihrer CCDSensoren in Zeilen- und Matrixkameras eingeteilt werden.

\subsection{Zeilenkameras}

Bei Zeilenkameras sind die CCD-Sensoren zeilenförmig angeordnet. Durch Aneinanderreihung einzelner Zeilen entsteht ein beliebig langer, konstant breiter Bildstreifen (s. Abb. 1).

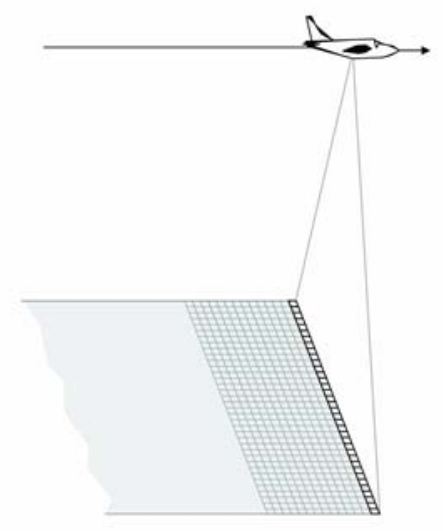

Abbildung 1: Zeilenweise Erfassung der Erdoberfläche mit einer Zeilenkamera (Bildstreifen) 
Die äußere Orientierung ändert sich aufgrund der dynamischen Bildaufzeichnung von Zeile zu Zeile. Daher ist die hochgenaue Aufzeichnung der sechs Orientierungsparameter (x,y, z, $\omega, \varphi, x)$ mit GPS (Global Positioning System) und INS (Inertialnavigationssystem) zwingend erforderlich. Ohne diese Parameter ist eine Zeilenorientierung nicht möglich.

Meist wird eine Dreizeilenkamera eingesetzt. Durch geneigte Objektive werden gleichzeitig zum Nadirblick ein Vorblick (z. B. ADS40: 28, $4^{\circ}$ ) und ein Rückblick (ADS40: $14,2^{\circ}$ ) aufgenommen. Für eine korrekte Orientierung muss Spezialsoftware eingesetzt werden. Durch optische Filter können Farbkanäle getrennt werden, die auf zusätzliche schmalbandige Sensorzeilen geleitet werden.

Nach der Zeilenkorrektur (s. Abb. 2) liegen die Bildstreifen in einer zentralperspektivischen Abbildung quer zur Flugrichtung vor. Diese Daten sind stereoskopisch betrachtbar. Nach erfolgter Orthorektifizierung (s. Kap. 6) können die Bilder als geometrisch entzerrte Orthophotos verwendet werden.

Eine Übersicht über technische Parameter aktueller Zeilenkameras zeigt Tabelle 1.

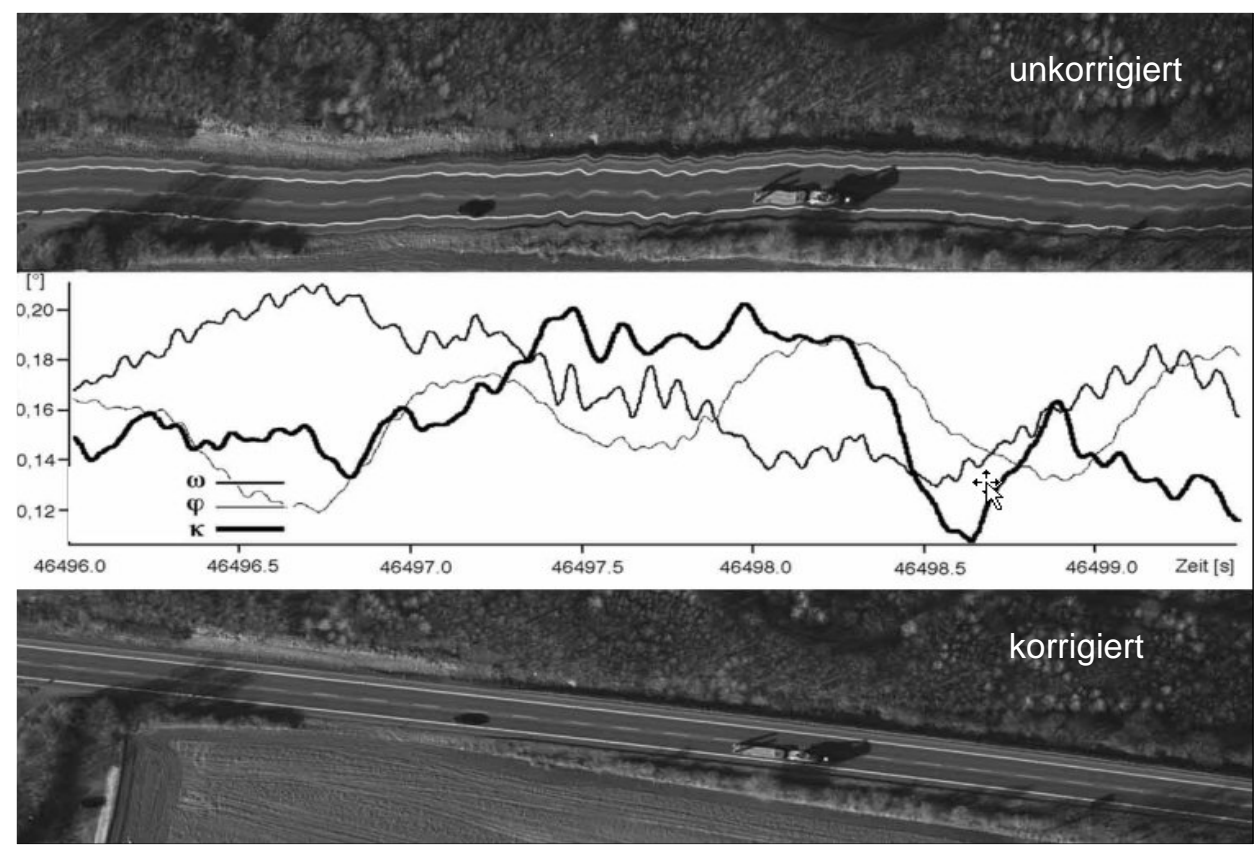

Abbildung 2: Korrektur der Winkelwerte bei einem Zeilenkamerabild (Quelle: ALBERTZ et al. 2002) 
Tabelle 1: $\quad$ Technische Parameter aktueller Zeilenkameras der Firma Leica Geosystems

\begin{tabular}{|c|c|c|c|}
\hline & & ADS40-SH40 & $\begin{array}{l}\text { ADS40-SH52 } \\
\text { ADS80-SH82 }\end{array}$ \\
\hline Pixelgröße & & 6,5 & 6,5 \\
\hline $\begin{array}{l}\text { Sensorgröl } \\
\text { Länge/ Bre }\end{array}$ & $(\mathrm{mm})$ & Variabel / 78 & Variabel / 78 \\
\hline Bildformat & nzahl Pixel) & $12.000 \times$ Streifenlänge & $12.000 \times$ Streifenlänge \\
\hline Farbtiefe ( & & 12 & 12 \\
\hline Brennweit & $\mathrm{nm})$ & 62,7 & 62,7 \\
\hline Focaleben & & $\begin{array}{l}\text { Pan Back } 14^{\circ} \\
\text { Pan Forward } 28^{\circ} \\
\text { RGB } 0^{\circ} \\
\text { Red Forward } 14^{\circ} \\
\text { Green Forward } 16^{\circ} \\
\text { NIR Forward } 18^{\circ}\end{array}$ & $\begin{array}{l}\text { Pan Back } 14^{\circ} \\
\text { Pan Forward } 27^{\circ} \\
\text { Pan Forward } 2^{\circ} \\
\text { RGB/NIR } 0^{\circ} \\
\text { RGB/NIR Backward } 16^{\circ} \\
-\end{array}$ \\
\hline 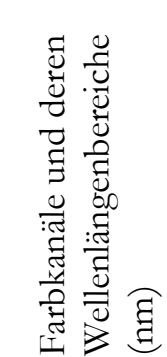 & $\begin{array}{l}\text { Blau } \\
\text { Grün } \\
\text { Rot } \\
\text { NIR }\end{array}$ & $\begin{array}{l}430-490 \\
535-585 \\
610-660 \\
835-885 \\
465-680\end{array}$ & $\begin{array}{l}428-492 \\
533-587 \\
608-662 \\
833-887 \\
465-680\end{array}$ \\
\hline
\end{tabular}

\subsection{Matrixkameras}

Bei Matrixkameras sind die CCD-Sensoren flächig in einer Matrix angeordnet. Wie bei analogen Luftbildern entstehen rechteckige, zentralperspektivische Aufnahmen mit einer Anordnung der Pixel in Zeilen und Spalten (s. Abb. 3). Diese können mit herkömmlicher photogrammetrischer Software bearbeitet und ausgewertet werden.

Während bei analogen Luftbildern das Bildkoordinatensystem durch die Bildrahmenmarken bestimmt wird, sind bei digitalen Luftbildern für die Bildorientierung Informationen über die Matrixgröße (Sensorgröße), die Anzahl der Pixel in $\mathrm{x}$ - und $\mathrm{y}$-Richtung oder die Pixelgröße erforderlich (s. Kap. 5). Diese Angaben können den Kalibrierungsprotokollen der verwendeten Luftbildkameras entnommen werden (s. Abb. 4). 


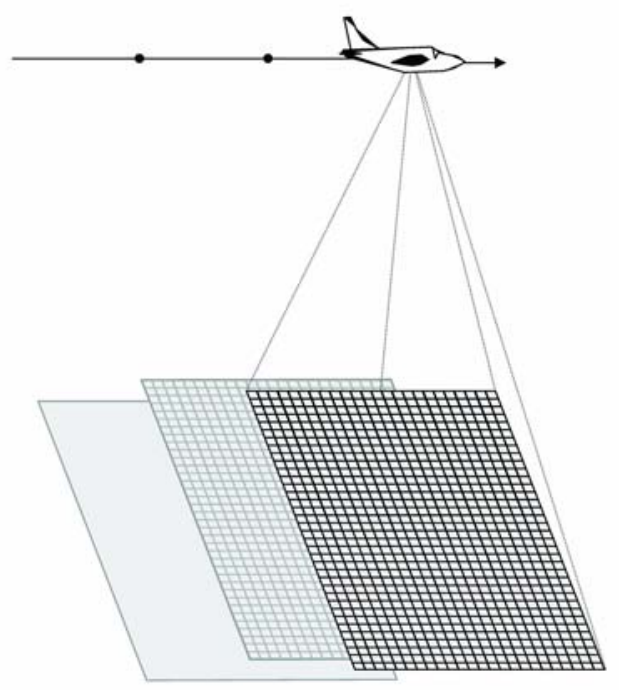

Abbildung 3: Flächige Erfassung der Erdoberfläche mit einer Matrixkamera (Einzelbilder)

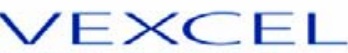

UltraCamXp, Serial Number UC-SXp-1-90513137 I M A G I N

\section{Panchromatic Camera}

\section{Large Format Panchromatic Output Image}

\begin{tabular}{|l|l|r|r|}
\hline Image Format & long track & $67.860 \mathrm{~mm}$ & 11310pixel \\
\hline & cross track & $103.860 \mathrm{~mm}$ & 17310 pixel \\
\hline & & & \\
\hline Image Extent & & $(-33.91,-51.95) \mathrm{mm}$ & $(33.91,51.95) \mathrm{mm}$ \\
\hline & & & \\
\hline Pixel Size & & $6.000 \mu \mathrm{m}^{*} 6.000 \mu \mathrm{m}$ & \\
\hline & & & $\pm 0.002 \mathrm{~mm}$ \\
\hline Focal Length & ck & $100.500 \mathrm{~mm}$ & $\pm 0.002 \mathrm{~mm}$ \\
\hline & & $0.000 \mathrm{~mm}$ & $\pm 0.002 \mathrm{~mm}$ \\
\hline Principal Point & X_ppa & $0.180 \mathrm{~mm}$ & \\
\hline (Level 2) & Y_ppa & & \multicolumn{2}{|c|}{} \\
\hline & & &
\end{tabular}

Abbildung 4: Ausschnitt aus dem Kalibrierungsprotokoll einer UltraCamXp 
Für die Triangulation und die Stereoauswertung wird eine Überlappung der Bilder in Flugrichtung benötigt. Dabei entscheiden die Fluggeschwindigkeit und die Bildwiederholungsrate über den Grad der Überlappung.

Matrixkameras können nach folgenden Bildformaten unterteilt werden:

- Großformatkameras: Basislänge des Sensors größer $10 \mathrm{~cm}$

(größer 11.000 Pixel x 7.500 Pixel)

- Mittelformatkameras: Basislänge des Sensors zwischen 4 und $10 \mathrm{~cm}$

(um 7.200 Pixel x 5.400 Pixel)

- Kleinformatkameras: Basislänge des Sensors kleiner $4 \mathrm{~cm}$

(kleiner 5.000 Pixel x 3.300 Pixel)

\subsubsection{Großformatkameras}

Großformatkameras werden für großräumige Befliegungen aufgrund ihrer hohen Flächenabdeckung eingesetzt. Die Kameras zeichnen sich durch eine hohe geometrische Genauigkeit aus. Die höchste Auflösung wird im panchromatischen Kanal (PAN) erreicht. Durch zusätzliche, kleinere Sensormatrizen und den Einsatz trennender optischer Systeme können verschiedene Farbkanäle in den Bereichen Rot (R), Grün (G), Blau (B) und Nahes Infrarot (NIR) aufgezeichnet werden. Damit die entstehenden Bilder die gleiche räumliche Abdeckung haben wie die PANBasisdaten, ist die Brennweite deutlich kürzer (Weitwinkel). Dies ist bei der Triangulation von Farbkanal-Rohdaten zu beachten. Durch Bildfusion werden die Farbrohdaten auf die höhere Auflösung der PAN-Basisdaten gerechnet (Pansharpening). Mit diesen Daten wird überwiegend gearbeitet.

Durch die sehr hohen Anschaffungskosten werden diese Kameras nur von professionellen Firmen eingesetzt. Derzeit sind zwei Kamerasysteme in diesem Bereich operationell verfügbar: DMC von Intergraph Z/I Imaging (s. Tab. 2) und UltraCam von Microsoft/Vexcel Imaging (s. Tab. 3). 
Tabelle 2: $\quad$ Technische Parameter aktueller Großformatkameras der Firma Intergraph Z/I Imaging

\begin{tabular}{|c|c|c|c|c|c|c|c|c|c|c|c|c|}
\hline 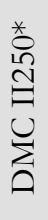 & $\begin{array}{l}\sigma_{0} \\
\text { L }\end{array}$ & 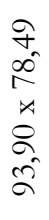 & 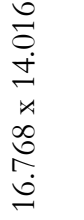 & 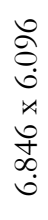 & $\Xi$ & 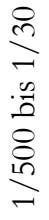 & 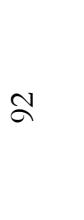 & $\stackrel{\operatorname{n}}{+}$ & 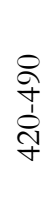 & 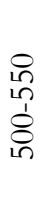 & $\begin{array}{l}8 \\
\varnothing \\
0 \\
8 \\
8 \\
0\end{array}$ & $\begin{array}{l}\stackrel{0}{\sim} \\
\infty \\
1 \\
0 \\
\stackrel{1}{1}\end{array}$ \\
\hline 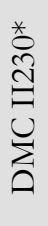 & $\begin{array}{l}{ }_{0}^{\prime} \\
\text { in }\end{array}$ & 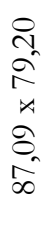 & 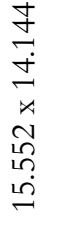 & $\begin{array}{l}0 \\
8 \\
0 \\
0 \\
x \\
1 \\
0 \\
+ \\
0 \\
0\end{array}$ & \pm & 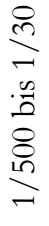 & $\curvearrowright$ & $\stackrel{2}{+}$ & 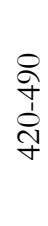 & 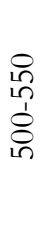 & $\begin{array}{l}0 \\
\text { ర } \\
1 \\
8 \\
8 \\
0\end{array}$ & 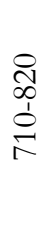 \\
\hline 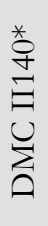 & $\stackrel{N}{N}$ & 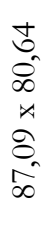 & 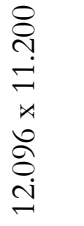 & $\begin{array}{l}0 \\
\stackrel{2}{0} \\
0 \\
\dot{1} \\
4 \\
0 \\
+ \\
0 \\
0 \\
0\end{array}$ & $\stackrel{\Xi}{\sqsupset}$ & 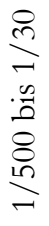 & $\curvearrowright$ & $\stackrel{\llcorner}{+}$ & 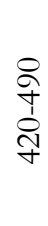 & 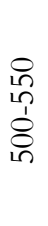 & 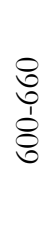 & 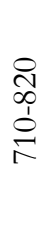 \\
\hline 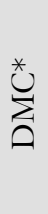 & $\stackrel{0}{\stackrel{0}{I}}$ & 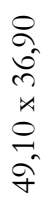 & 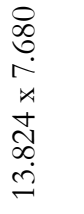 & 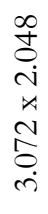 & $\stackrel{\sim}{\sim}$ & $\begin{array}{l}0 \\
0 \\
7 \\
\frac{n}{0} \\
0 \\
8 \\
0 \\
10 \\
7\end{array}$ & $\underset{\sim}{\sim}$ & $\stackrel{\llcorner}{\sim}$ & $\begin{array}{l}8 \\
\infty \\
10 \\
\delta \\
\delta\end{array}$ & 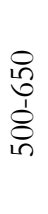 & 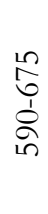 & 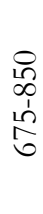 \\
\hline 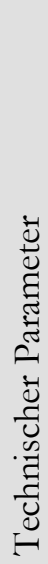 & 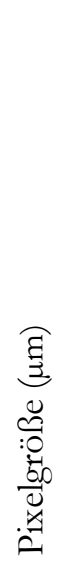 & 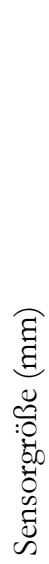 & 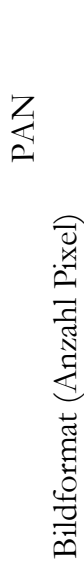 & $\begin{array}{l}\text { Z } \\
\text { Z } \\
\text { ô } \\
\sim 1\end{array}$ & 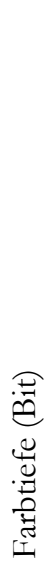 & 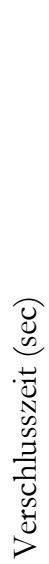 & 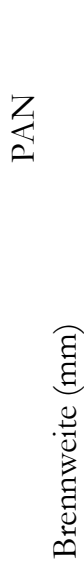 & $\frac{\mathscr{b}}{Z}$ & $\frac{\vec{\sigma}}{m}$ & 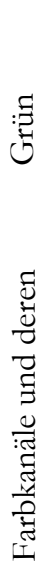 & छ్ & 当 \\
\hline
\end{tabular}

* Technische Angaben der Firma Intergraph Z/I Deutschland GmbH, Stand August 2011 
Tabelle 3: Technische Parameter aktueller Großformatkameras der Firma Microsoft/Vexcel Imaging

\begin{tabular}{|c|c|c|c|c|c|c|c|c|c|c|c|c|}
\hline 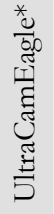 & ก̂ & 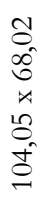 & 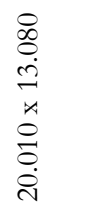 & 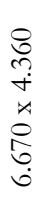 & \pm & $\begin{array}{l}\sqrt{2} \\
2 \\
0 \\
0 \\
8 \\
0 \\
0 \\
-1\end{array}$ & $\frac{\stackrel{0}{\stackrel{N}{ }}}{\stackrel{\infty}{\infty}}$ & $\frac{尺}{\frac{R}{N}}$ & 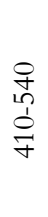 & 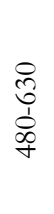 & $\begin{array}{l}8 \\
\stackrel{8}{1} \\
\infty \\
\infty \\
i n\end{array}$ & $\begin{array}{l}8 \\
\frac{8}{0} \\
\frac{1}{\delta}\end{array}$ \\
\hline 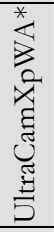 & $\hat{\sigma}_{0}$ & $\begin{array}{l}\infty \\
\infty \\
\hat{b} \\
\dot{1} \\
\infty \\
\infty \\
0 \\
0\end{array}$ & 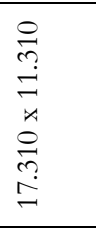 & 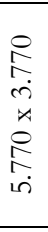 & $\stackrel{\sim}{\wedge}$ & 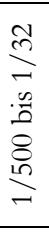 & $\stackrel{R}{R}$ & $\hat{\imath}$ & 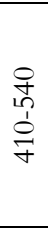 & 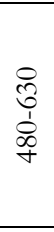 & $\begin{array}{l}\stackrel{8}{2} \\
\stackrel{1}{1} \\
\infty \\
\text { in }\end{array}$ & $\begin{array}{l}8 \\
\frac{8}{1} \\
\frac{1}{\delta}\end{array}$ \\
\hline 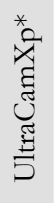 & $\hat{O}_{0}$ & $\begin{array}{l}0 \\
\infty \\
\hat{\sigma} \\
4 \\
0 \\
\infty \\
\infty \\
0 \\
0\end{array}$ & 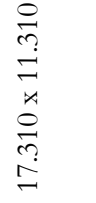 & $\begin{array}{l}\stackrel{R}{\hat{t}} \\
\dot{r} \\
\dot{x} \\
\stackrel{2}{R} \\
\text { in }\end{array}$ & $\stackrel{\sim}{\wedge}$ & $\begin{array}{l}\frac{1}{2} \\
\frac{0}{2} \\
0 \\
0 \\
0 \\
0 \\
-1\end{array}$ & $\stackrel{8}{\&}$ & 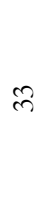 & 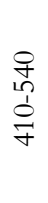 & 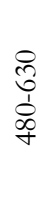 & 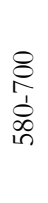 & $\begin{array}{l}8 \\
\frac{8}{0} \\
\stackrel{5}{0}\end{array}$ \\
\hline 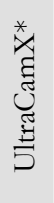 & $\stackrel{N}{N}$ & $\begin{array}{l}1 \\
\infty \\
\hat{\sigma} \\
x \\
\infty \\
\infty \\
\infty \\
0\end{array}$ & 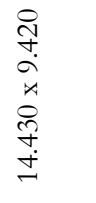 & 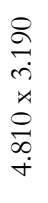 & $\stackrel{\sim}{\wedge}$ & \begin{tabular}{l}
$\sqrt{2}$ \\
\multirow{2}{n}{} \\
0 \\
0 \\
8 \\
0 \\
0 \\
-1
\end{tabular} & $\underset{\sim}{\stackrel{2}{2}}$ & 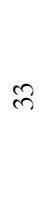 & 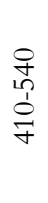 & 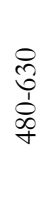 & \begin{tabular}{l}
8 \\
\multirow{2}{1}{} \\
1 \\
$\infty$ \\
$i n$
\end{tabular} & $\begin{array}{l}\stackrel{8}{8} \\
\frac{1}{1} \\
\text { هे }\end{array}$ \\
\hline 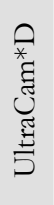 & $\stackrel{\circ}{\circ}$ & 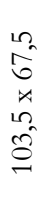 & 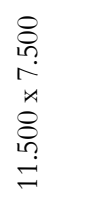 & 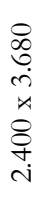 & $\stackrel{\sim}{\sim}$ & $\begin{array}{l}\sqrt{2} \\
-1 \\
0 \\
0 \\
8 \\
8 \\
0 \\
-1\end{array}$ & 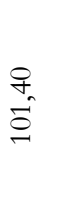 & $\stackrel{\infty}{\sim}$ & $\begin{array}{l}\text { के } \\
\text { గn } \\
\text { ळे }\end{array}$ & $\begin{array}{l}8 \\
\stackrel{0}{0} \\
\stackrel{1}{8} \\
\text { f }\end{array}$ & 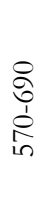 & $\begin{array}{l}\text { Pे } \\
\text { ì } \\
\text { 엉 }\end{array}$ \\
\hline 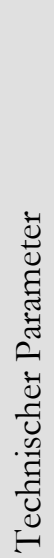 & 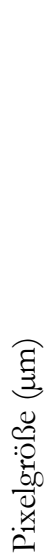 & 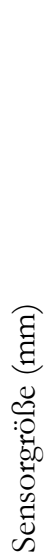 & 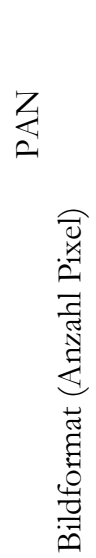 & 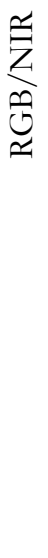 & 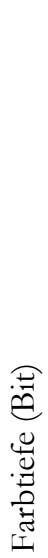 & 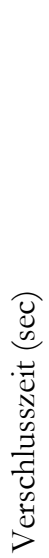 & Z & 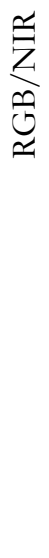 & $\frac{\vec{\Xi}}{\infty}$ & 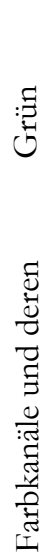 & $\widehat{\Xi}$ & 自 \\
\hline
\end{tabular}

* Technische Angaben der Firma Microsoft/Vexcel Imaging, Stand August 2011

Beiträge aus der NW-FVA, Band 7, 2012 


\subsubsection{Mittelformatkameras}

Mittelformatkameras werden bei kleinen und mittleren Projekten eingesetzt, um ein gutes Preis-Leistungs-Verhältnis zu erzielen. Die Flächenleistung ist zwar nicht ganz so hoch wie bei Großformatkameras. Die sonstigen technischen Eigenschaften, wie geometrische Genauigkeit und radiometrische Auflösung, sind jedoch mit Großformatkameras vergleichbar. Einige Mittelformatkameras arbeiten nur im 3Kanal-Modus. Das heißt, Bilddaten können entweder im RGB- oder im CIRModus aufgenommen werden. Die Kanalkombination muss vor dem Flug bestimmt werden. Es gibt deutliche Qualitätsunterschiede zwischen den einzelnen Modellen. Eine Auswahl aktueller Mittelformatkameras zeigt Tabelle 4.

\subsubsection{Kleinformatkameras}

Kleinformatkameras werden für kleinräumige Projekte in bemannten und auch unbemannten Flugkörpern eingesetzt. Sie besitzen eine geringe Flächenleistung. Es können alle handelsüblichen Digitalkameras eingesetzt werden. Vor der Verwendung für Luftbildaufnahmen sollten die Kameras kalibriert werden. Um eine ausreichende Überlappung der Bilder zu erreichen, muss eine schnelle Bildwiederholungsrate möglich sein.

Triangulationen sind auf der Basis einfacher technischer Angaben (Brennweite, Sensorgröße, Anzahl der Pixel und Pixelgröße) auch hier möglich. Es ist zu beachten, dass die eingebauten Linsensysteme häufig starke Verzeichnungen der Bilder bewirken, die eine Auswertung sehr stören oder sogar unmöglich machen können. Deshalb ist die anspruchvolle Aufbereitung (Orthophotos, Stereomodelle und Geländemodelle) Fachkundigen zu überlassen.

\subsection{Technische Parameter}

\subsubsection{Spektrale Auflösung}

Die spektrale Auflösung ist durch die Anzahl der Spektralkanäle, ihre Breite und ihre Wellenlängenunterschiede definiert.

Digitale Luftbildkameras zeichnen aktuell neben dem panchromatischen Kanal bis zu 4 Spektralkanäle auf (Rot, Grün, Blau, Nahes Infrarot). Gebräuchliche Wellenlängenbereiche je Farbkanal variieren von Kameratyp zu Kameratyp (s. Tab. $2,3$ und 4$)$. 
Tabelle 4: $\quad$ Technische Parameter aktueller Mittelformatkameras

\begin{tabular}{|c|c|c|c|c|c|c|c|c|c|c|c|c|}
\hline 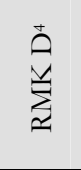 & $\stackrel{N}{n}$ & 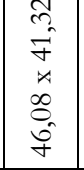 & 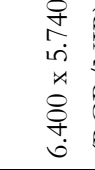 & 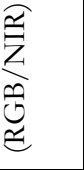 & \pm & $\begin{array}{l}\stackrel{n}{a} \\
0 \\
\stackrel{n}{n}= \\
=\end{array}$ & $\stackrel{\text { In }}{f}$ & $\nabla$ & $\begin{array}{l}8 \\
0 \\
0 \\
0 \\
0 \\
n\end{array}$ & 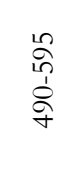 & $\begin{array}{l}\stackrel{0}{\rightarrow} \\
\infty \\
\infty \\
i n \\
i n\end{array}$ & 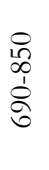 \\
\hline 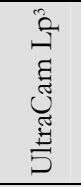 & $\hat{O}_{0}$ & 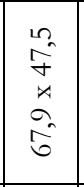 & 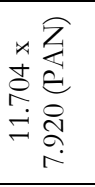 & 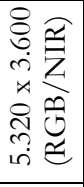 & $\stackrel{\sim}{\wedge}$ & $\begin{array}{l}\frac{n}{3} \\
\frac{n}{2} \\
\frac{n}{2}\end{array}$ & 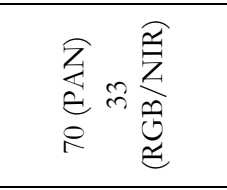 & + & 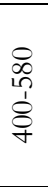 & 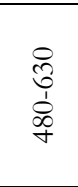 & $\begin{array}{l}0 \\
0 \\
0 \\
1 \\
0 \\
0 \\
i n\end{array}$ & $\begin{array}{l}8 \\
8 \\
\frac{1}{2} \\
8 \\
0\end{array}$ \\
\hline$\sum_{\substack{50 \\
0}}^{\substack{+\\
}}$ & $\hat{\sigma}_{0}$ & 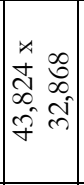 & 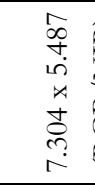 & 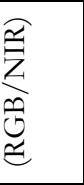 & $\stackrel{\odot}{\rightleftharpoons}$ & 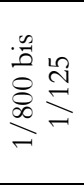 & 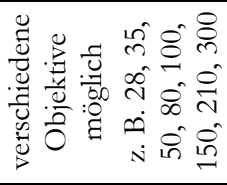 & $m$ & $\begin{array}{l}\text { q } \\
\text { Ln } \\
\stackrel{1}{8} \\
\text { q }\end{array}$ & $\begin{array}{l}8 \\
0 \\
\vdots \\
\text { o } \\
\text { o }\end{array}$ & $\begin{array}{l}8 \\
0 \\
0 \\
\infty \\
\infty \\
i n\end{array}$ & $\begin{array}{l}8 \\
8 \\
\frac{1}{1} \\
8 \\
\infty\end{array}$ \\
\hline$\sum_{\substack{50 \\
0}}^{\infty}$ & $\hat{\sigma}_{0}$ & 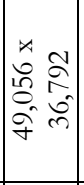 & 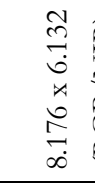 & 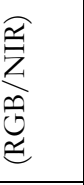 & $\stackrel{\odot}{\smile}$ & 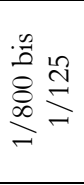 & 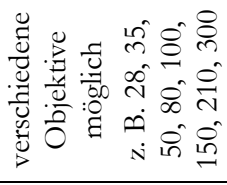 & $n$ & $\begin{array}{l}\text { ơ } \\
\text { 1े } \\
\text { ơ }\end{array}$ & \begin{tabular}{l}
8 \\
0 \\
+ \\
\multirow{1}{\infty}{} \\
\multirow{+}{*}{}
\end{tabular} & $\begin{array}{l}0 \\
0 \\
0 \\
\vdots \\
\infty \\
i n\end{array}$ & $\begin{array}{l}8 \\
8 \\
\frac{1}{1} \\
8 \\
\infty\end{array}$ \\
\hline 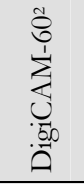 & $\hat{\sigma}_{0}$ & 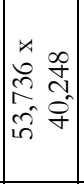 & 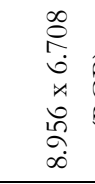 & 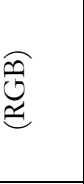 & $\stackrel{0}{-}$ & 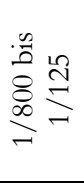 & 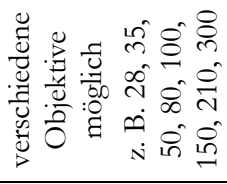 & $n$ & 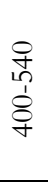 & $\begin{array}{l}8 \\
0 \\
0 \\
1 \\
\infty \\
+ \\
+\end{array}$ & $\begin{array}{l}8 \\
0 \\
i \\
\circ \\
\infty \\
\text { in }\end{array}$ & ' \\
\hline 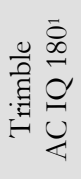 & in & 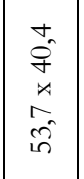 & 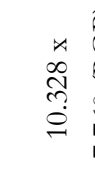 & 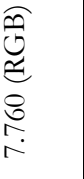 & $\stackrel{\odot}{\longrightarrow}$ & $\begin{array}{l}1 \\
2 \\
: 3 \\
0 \\
0 \\
0 \\
-1\end{array}$ & 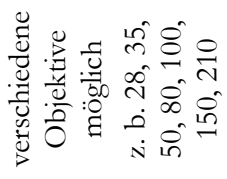 & $n$ & 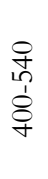 & 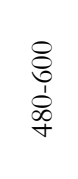 & $\begin{array}{l}8 \\
0 \\
0 \\
1 \\
\infty \\
i n\end{array}$ & 1 \\
\hline 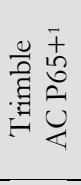 & $\hat{O}_{0}$ & $\begin{array}{l}\stackrel{+}{o} \\
\stackrel{q}{4} \\
\stackrel{\leftrightarrow}{\hat{n}}\end{array}$ & 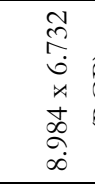 & 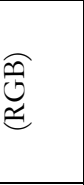 & $\stackrel{0}{-}$ & $\begin{array}{l}\overrightarrow{1} \\
: \frac{9}{0} \\
0 \\
0 \\
-1\end{array}$ & 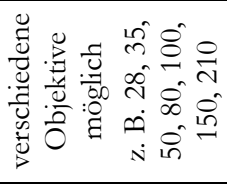 & $m$ & 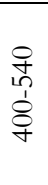 & 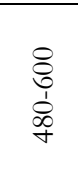 & $\begin{array}{l}0 \\
\stackrel{0}{0} \\
1 \\
\infty \\
\infty \\
i n\end{array}$ & 1 \\
\hline 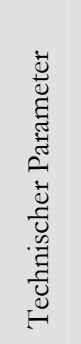 & 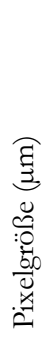 & 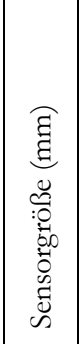 & 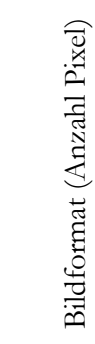 & & 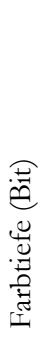 & 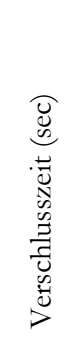 & 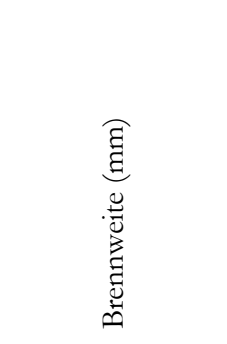 & 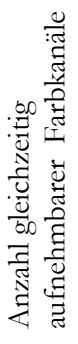 & $\frac{\vec{\Xi}}{m}$ & 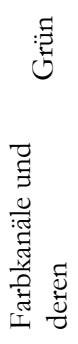 & 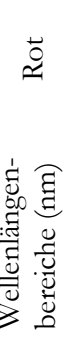 & 号 \\
\hline
\end{tabular}

${ }^{1}$ Technische Angaben der Firma Trimble, Stand August 2011

2 Technische Angaben der Firma IGI mbH, Stand August 2011

${ }^{3}$ Technische Angaben der Firma Microsoft/Vexcel Imaging, Stand August 2011

${ }^{4}$ Technische Angaben der Firma Intergraph Z/I Deutschland GmbH, Stand August 2011 


\subsubsection{Radiometrische Auflösung}

Die radiometrische Auflösung ist die Fähigkeit eines Sensors, Strahlungsunterschiede messtechnisch zu erfassen (DEUTSCHES INSTITUT FÜR NORMUNG 2011). In digitalen Bildern werden diese gemessenen Strahlungsunterschiede kanalweise innerhalb der kleinsten Bildeinheit (Pixel) in Form binärer Zahlenwerte gespeichert. Zur Visualisierung erfolgt eine Umsetzung in Grauwerte.

Jedes digitale Bild enthält einen festgelegten Wertebereich (Anzahl der Abstufungen je Bildkanal). So kann man zum Beispiel bei einer radiometrischen Auflösung von 8 Bit 256 Zahlenwerte unterscheiden. Haufig wird hierbei auch der Begriff der Farbtiefe verwendet.

Für digitale Stereoauswertungen sind erfahrungsgemäß 8 Bit-Bilddaten ausreichend, während bei automatisierten Klassifizierungsverfahren die volle radiometrische Auflösung z. B. von 12 Bit oder mehr verfügbar sein sollte. Allerdings ist dafür ein höherer Speicherbedarf zu berücksichtigen. Nicht jede Software kann 12 Bit-Bilddaten verarbeiten. In einem solchen Fall müssen die Bilddaten auf 8 Bit oder 16 Bit umgerechnet werden. Für die Archivierung empfiehlt sich aber grundsätzlich die bestmögliche Auflösung.

Wichtig für die Beurteilung der Qualität digitaler Luftbilder ist die Verteilung der Grauwerte. Diese wird in Histogrammen abgebildet. Dabei wird auf der yAchse die Anzahl der belegten Pixel und auf der x-Achse die Werteskala der Grauwerte dargestellt, z. B. bei 8 Bit-Daten Werte von 0 (schwarz) bis 255 (weiß) (s. Abb. 5).
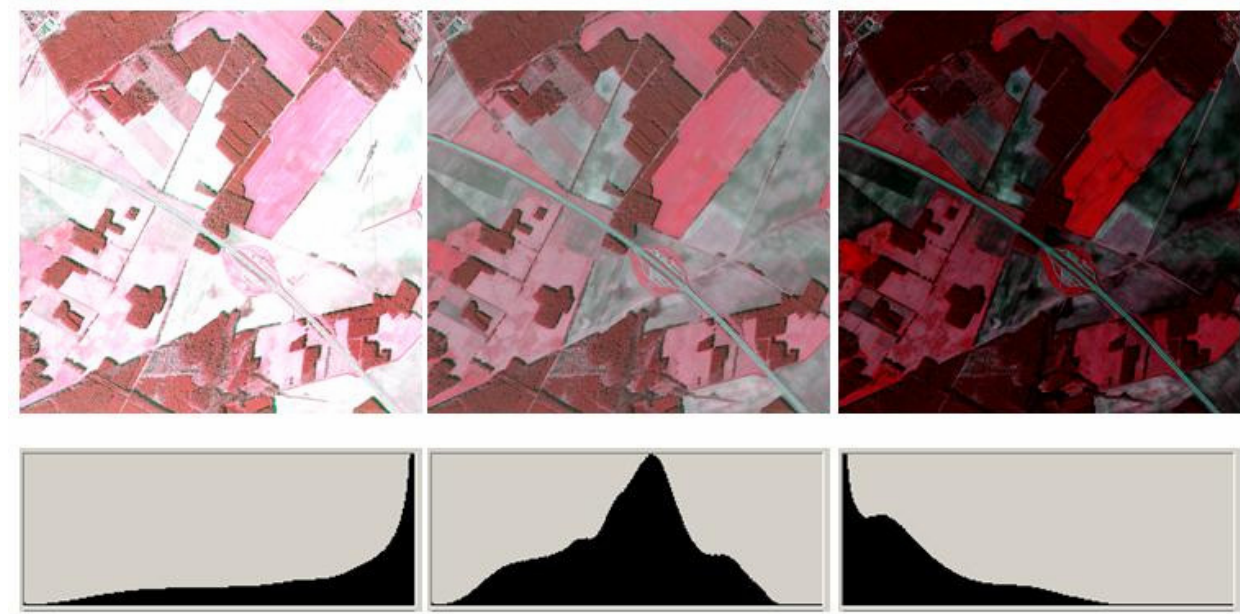

Abbildung 5: Histogramme unterschiedlich belichteter Luftbilder (links: Überbelichtung, Mitte: ausgewogene Belichtung, rechts: Unterbelichtung); $x$-Achse: Werteskala der Grauwertstufen; y-Achse: Anzabl von Pixeln in den einzelnen Grawwertstufen 
Die Grauwerte sollten über den gesamten Wertebereich verteilt sein und möglichst keine Sprünge, Leerbereiche oder starke Klumpungen an den Rändern aufweisen. Letzteres deutet auf Über- oder Unterbelichtung hin. Das Histogramm enthält allerdings keine Informationen, ob die Bilder bereits durch Schritte der digitalen Bildbearbeitung verändert wurden oder noch Originalbilder sind. Dies kann z. B. bei Zeitreihenuntersuchungen $\mathrm{zu}$ verzerrten Ergebnissen führen, wenn digitale Klassifizierungsverfahren angewandt werden (s. Kap. 7).

\subsubsection{Bodenauflösung und Abdeckung}

Die Bodenauflösung eines digitalen Luftbildes, auch ground sample distance (GSD) genannt, gibt die Größe eines Pixels in der realen Welt an. Sie lässt sich wie folgt berechnen:

$$
\mathrm{GSD}[\mathrm{m}]=\frac{\text { Flughöhe über Grund } \mathrm{h}_{\mathrm{G}}[\mathrm{m}]}{\text { Brennweite c }[\mathrm{mm}]} \times \text { Kantenlänge eines Pixels }[\mathrm{mm}]
$$

Die Größe des abgebildeten Geländeausschnittes (Abdeckung) hängt von der Pixel- und Sensorgröße der verwendeten Kamera ab und variiert entsprechend (s. Tab. 5).

Die Wahl der Bodenauflösung hängt von der Größe und Struktur der kleinsten $\mathrm{zu}$ erfassenden Objekte $\mathrm{ab}$ und ob diese detektiert, erkannt oder identifiziert werden sollen. Dabei werden die drei Entscheidungsniveaus wie folgt definiert (SANDAU 2005):

- Detektieren ist das Entdecken der Existenz eines Objektes (z. B. Baum)

- Erkennen ist das Zuordnen eines Objektes zu einer Typengruppe (z. B. Baumart)

- Identifizieren ist die eindeutige Erkennung der Beschaffenheit eines Objektes (z. B. Schaden)

Je detaillierter ausgewertet werden soll, desto mehr Pixel müssen ein Objekt repräsentieren. Dabei bedeutet eine höhere als die notwendige Auflösung keinen objektbezogenen Informationsgewinn.

Für die Erkennung von Objekten kann die optimale Bodenauflösung durch folgende Faustformel bestimmt werden (nach SANDAU 2005):

$$
\mathrm{GSD}[\mathrm{m}]=\frac{\text { Größe des kleinsten zu erkennenden Objektes }[\mathrm{m}]}{3}
$$

Für die Identifizierung von Objekten kann die Bodenauflösung ebenfalls durch eine Faustformel bestimmt werden (nach SANDAU 2005):

$$
\mathrm{GSD}[\mathrm{m}]=\frac{\text { Größe des kleinsten zu identifizierenden Objektes }[\mathrm{m}]}{21}
$$


Tabelle 5: Bodenauflösung und Bildabdeckung verschiedener Luftbildkameras bei einer Flughöhe von 2.000 m über Grund

\begin{tabular}{|c|c|c|c|c|c|c|}
\hline & $\begin{array}{l}\text { Anzahl } \\
\text { der Pixel } \\
\text { Länge / } \\
\text { Breite }\end{array}$ & $\begin{array}{l}\text { Größe des } \\
\text { Sensors } \\
\text { (mm) } \\
\text { Länge / } \\
\text { Breite }\end{array}$ & $\begin{array}{l}\text { Größe } \\
\text { eines } \\
\text { Pixel } \\
(\mu \mathrm{m})\end{array}$ & $\begin{array}{l}\text { Brenn- } \\
\text { weite } \\
\text { (mm) }\end{array}$ & $\begin{array}{l}\text { Boden- } \\
\text { auflösung } \\
\text { (m) }\end{array}$ & $\begin{array}{c}\text { Bild- } \\
\text { abdeckung } \\
\text { (m) } \\
\text { Länge / } \\
\text { Breite }\end{array}$ \\
\hline \multicolumn{7}{|c|}{ Großformatkamera } \\
\hline $\mathrm{DMC}(\mathrm{PAN})$ & $\begin{array}{c}13.824 \mathrm{x} \\
7.680\end{array}$ & $\begin{array}{c}49,10 \mathrm{x} \\
36,90\end{array}$ & 12 & 120 & 0,20 & $\begin{array}{c}2.764,8 \mathrm{x} \\
1.536\end{array}$ \\
\hline $\begin{array}{l}\mathrm{DMC} \\
\text { (RGB/NIR) }\end{array}$ & $\begin{array}{c}3.072 \mathrm{x} \\
2.048\end{array}$ & $\begin{array}{c}49,10 \mathrm{x} \\
36,90\end{array}$ & 12 & 25 & 0,96 & $\begin{array}{c}2.949 \mathrm{x} \\
1.966\end{array}$ \\
\hline $\begin{array}{l}\text { UltraCamXp } \\
\text { (PAN) }\end{array}$ & $\begin{array}{c}17.310 \mathrm{x} \\
11.310\end{array}$ & $\begin{array}{c}103,86 \mathrm{x} \\
67,89\end{array}$ & 6 & 100 & 0,12 & $\begin{array}{c}2.077 \mathrm{x} \\
1.357\end{array}$ \\
\hline $\begin{array}{l}\text { UltraCamXp } \\
\text { (RGB/NIR) }\end{array}$ & $\begin{array}{c}5.770 \mathrm{x} \\
3.770\end{array}$ & $\begin{array}{c}103,86 \mathrm{x} \\
67,89\end{array}$ & 6 & 33 & 0,36 & $\begin{array}{c}2.077 \mathrm{x} \\
1.357\end{array}$ \\
\hline \multicolumn{7}{|c|}{ Mittelformatkamera } \\
\hline $\begin{array}{l}\text { Trimble } \\
\text { AIC P65+ }\end{array}$ & $\begin{array}{c}8.984 x \\
6.732\end{array}$ & $\begin{array}{c}53,90 \times \\
40,40\end{array}$ & 6 & z. B. 50 & 0,24 & $\begin{array}{c}2.156 \mathrm{x} \\
1.616\end{array}$ \\
\hline DigiCAM-50 & $\begin{array}{c}8.176 \mathrm{x} \\
6.132\end{array}$ & $\begin{array}{c}49,09 \mathrm{x} \\
36,79\end{array}$ & 6 & z. B. 50 & 0,24 & $\begin{array}{c}1.962 \mathrm{x} \\
1.472\end{array}$ \\
\hline $\begin{array}{l}\text { UltraCamLp } \\
(\mathrm{PAN})\end{array}$ & $\begin{array}{c}11.704 \mathrm{x} \\
7.920\end{array}$ & $\begin{array}{c}67,90 \mathrm{x} \\
47,50\end{array}$ & 6 & 70 & 0,17 & $\begin{array}{c}1.990 \mathrm{x} \\
1.346\end{array}$ \\
\hline $\begin{array}{l}\text { UltraCamLp } \\
\text { (RGB/NIR) }\end{array}$ & $\begin{array}{c}5.320 \mathrm{x} \\
3.600\end{array}$ & $\begin{array}{c}67,90 x \\
47,50\end{array}$ & 6 & 33 & 0,36 & $\begin{array}{c}1.915 \mathrm{x} \\
1.296\end{array}$ \\
\hline \multicolumn{7}{|c|}{ Zeilenkamera } \\
\hline ADS80 & $\begin{array}{c}\text { variabel } \\
\text { (flugbahn- } \\
\text { abhängig)/ } \\
12.000\end{array}$ & $\begin{array}{c}\text { variabel / } \\
78\end{array}$ & 6,5 & 62,77 & 0,19 & $\begin{array}{c}\text { variabel/ } \\
2.520\end{array}$ \\
\hline
\end{tabular}

\subsubsection{Dateigrößen}

Die Dateigröße eines Bildes ist abhängig von der radiometrischen Auflösung und der Größe des Sensors. Ein Schwarzweiß-Bild hat üblicherweise eine Auflösung von 8 Bit, ein Dreikanal-Farbbild (z. B. RGB) 3 mal 8 Bit, also 24 Bit. Pro Pixel müssen also bei Schwarzweiß-Bildern genau 1 Byte und bei Farbbildern 3 Bytes auf der Festplatte gespeichert werden. Bei 16 Bit-Bildern verdoppelt sich die Dateigröße je Farbkanal. Die Dateigröße in Bytes ergibt sich somit bei SW-Bildern aus 
der Anzahl der Bildpixel. Bei Farbbildern ist die Dateigröße n mal so groß wie die Anzahl der Pixel ( $\mathrm{n}=$ Anzahl der Farbkanäle).

Tabelle 6 zeigt die Dateigrößen von Rohdaten gebräuchlicher Matrixkameras.

Tabelle 6: Dateigrößen unkomprimierter Robdaten gebräuchlicher Matrixkameras

\begin{tabular}{|c|c|c|c|c|c|c|}
\hline & \multicolumn{3}{|c|}{ Großformatkameras } & \multicolumn{3}{|c|}{ Mittelformatkameras } \\
\hline & DMC & $\begin{array}{c}\text { UltraCam } \\
\mathrm{Xp}\end{array}$ & $\begin{array}{l}\text { UltraCam } \\
\text { Xp WA }\end{array}$ & $\begin{array}{l}\text { Trimble } \\
\text { AC P65+ }\end{array}$ & $\begin{array}{l}\text { DigiCAM- } \\
50\end{array}$ & $\begin{array}{c}\text { UltraCam } \\
\text { Lp }\end{array}$ \\
\hline $\begin{array}{l}\text { Pixelgröße } \\
(\mu \mathrm{m})\end{array}$ & 12 & 6 & 6 & 6 & 6 & 6 \\
\hline $\begin{array}{l}\text { Sensorgröße } \\
(\mathrm{mm})\end{array}$ & $\begin{array}{c}49,10 x \\
36,90\end{array}$ & $\begin{array}{c}103,86 \mathrm{x} \\
67,86\end{array}$ & $\begin{array}{c}103,86 \mathrm{x} \\
67,86\end{array}$ & $\begin{array}{c}53,9 x \\
40,4\end{array}$ & $\begin{array}{c}49,05 x \\
36,79\end{array}$ & $\begin{array}{c}67,9 x \\
47,5\end{array}$ \\
\hline$\widehat{\vartheta}$ PAN & $\begin{array}{c}13.824 \mathrm{x} \\
7.680\end{array}$ & $\begin{array}{c}17.310 \mathrm{x} \\
11.310\end{array}$ & $\begin{array}{c}17.310 \mathrm{x} \\
11.310\end{array}$ & $\begin{array}{c}8.984 x \\
6.732\end{array}$ & $\begin{array}{c}8.176 x \\
6.132\end{array}$ & $\begin{array}{c}11.704 \mathrm{x} \\
7.920\end{array}$ \\
\hline 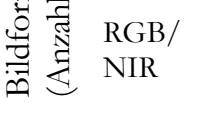 & $\begin{array}{c}3.072 \mathrm{x} \\
2.048\end{array}$ & $\begin{array}{c}5.770 \mathrm{x} \\
3.770\end{array}$ & $\begin{array}{c}5.770 \mathrm{x} \\
3.770\end{array}$ & $\begin{array}{c}8.984 x \\
6.732\end{array}$ & $\begin{array}{c}8.176 x \\
6.132\end{array}$ & $\begin{array}{c}5.320 x \\
3.600\end{array}$ \\
\hline $\begin{array}{l}\text { Dateigröße } \\
\text { für den } 8 \text { Bit- } \\
\text { PAN Kanal } \\
\text { (MB) }\end{array}$ & 101 & 187 & 187 & 58 & 48 & 88 \\
\hline $\begin{array}{l}\text { Dateigröße je } \\
8 \text { Bit-Farb- } \\
\text { kanal } \\
\text { (MB) }\end{array}$ & 6 & 21 & 21 & 58 & 48 & 18 \\
\hline
\end{tabular}

Bei Zeilenkameras ist die Dateigröße abhängig von der Länge des Flugstreifens, da hier im Gegensatz zu Matrixkameras keine Einzelbilder, sondern kontinuierliche Flugstreifen aufgezeichnet werden. 



\section{Digitalisierung analoger Luftbilder}

Luftbilder werden hier als photogrammetrische Produkte verstanden, deren Digitalisierung je nach Verwendungszweck besondere, meist sehr hohe Ansprüche sowohl an den Scanner als auch an das Personal stellt. Daher ist es sinnvoll, die Digitalisierung von Luftbildern von Firmen durchführen zu lassen, welche über eine entsprechende technische Ausstattung, wie z. B. einen Präzisionsscanner, und das dazugehörige Know-how verfügen. Dennoch ist es für die Vergabe solcher Arbeiten und die Qualitätskontrolle wichtig, sich mit den Grundlagen des Digitalisierungsvorganges vertraut zu machen.

Dieses Kapitel gibt einen Überblick über Grundbegriffe, Technik und die beim Digitalisieren analoger Luftbilder entstehenden Daten.

Weitere detaillierte Vorgaben und technische Beschreibungen können der Deutschen Norm DIN 18740-2 „Photogrammetrische Produkte - Anforderungen an das gescannte Luftbild“ entnommen werden.

\subsection{Scannertypen}

Analoge Luftbilder werden mit Hilfe von Scannern in digitale Form gebracht. Dabei wird durch optisches Abtasten ein Rasterbild erzeugt, das aus regelmäßig angeordneten Pixeln (Bildpunkten) besteht und als Abbild der Vorlage digital gespeichert wird.

Photogrammetrische Auswertungen und Messungen erfordern den Einsatz von photogrammetrischen Scannern, die beim Abtastvorgang nur geringe Bildverzerrungen bewirken. Sie werden regelmäßig kalibriert und weisen einen maximalen geometrischen Fehler von $10 \mu \mathrm{m}$ auf (DEUTSCHES INSTITUT FÜr NORMUNG 2005).

Für semantische Anwendungen und einfache Interpretationszwecke reicht hingegen häufig auch ein herkömmlicher Büroscanner. Dieser muss die Möglichkeit bieten, Dias von mindestens $23 \times 23 \mathrm{~cm}$ Größe zu scannen, was mindestens ein DIN A3-Format erfordert. Beim Scanvorgang können jedoch geometrische oder radiometrische Fehler entstehen, mit denen das digitale Bild dann behaftet ist. Diese Verzerrungen können kontrolliert und bei der späteren Orientierung der Bilder berücksichtigt werden. Dazu wird eine mit einer Folie überzogene maßhaltige Aluplatte verwendet, worauf mittels eines Orthogonal-Koordinatografen Punkte im $5 \mathrm{~cm}$-Raster präzise aufgetragen werden. Die Kalibrierungsplatte wird während des Scannens der Luftbilder periodisch eingelegt. Mit Hilfe einer geeigneten Photogrammetriesoftware können die Koordinaten der Punkte nach dem Scanvorgang gemessen und mit ihrer Soll-Lage verglichen werden. Die so ermittelten Verzerrungen können dann mit affinen Parametern modelliert und somit bei 
der Rekonstruktion der inneren Orientierung der Luftbildkamera weitgehend korrigiert werden.

Ist eine Kalibrierung der Scanner nicht möglich, sollten für qualitativ hochwertige Auswertungen keine Büroscanner verwendet werden.

\subsection{Bildauflösung und Dateigrößen}

Bei der Verarbeitung digitaler Bilder stellt die Auflösung einen sehr wichtigen Faktor dar. Feiner werdende Auflösungen steigern den Informationsgehalt bis zu dem Zeitpunkt, an dem die Auflösung des Scans die der Vorlage erreicht. Allerdings nehmen die entstehenden Datenmengen gleichzeitig exponentiell zu.

Für die Wahl der passenden digitalen Auflösung spielen folgende Faktoren eine Rolle:

- Auflösung des analogen Filmmaterials

- Anforderungen, die sich aus dem Auswertungszweck ergeben

- Farbtiefe / Anzahl der Grauwerte (8/12/16 Bit)

- Anfallende Datenmenge pro Bild

Bei Luftbildscans wird die Auflösung meist in Mikrometer $(\mu \mathrm{m})$ angegeben, bei Scannern und Druckern in dpi (dots per inch). Beide Angaben beschreiben die Größe der Pixel eines Digitalbildes. Der Wert in $\mu \mathrm{m}$ bezeichnet die Kantenlänge eines Pixels, dpi gibt an, wie viele Pixel einen Inch füllen.

Die Auflösung von analogem Filmmaterial wird oft in Linien (l) oder Linienpaaren (lp) pro Millimeter angegeben. Beide Bezeichnungen sind identisch und werden synonym verwendet. Im Folgenden werden einige Hilfsmittel zur Umrechnung gängiger Maßeinheiten aufgelistet:

$1 \mu \mathrm{m}=0,001 \mathrm{~mm}$

$1.000 \mu \mathrm{m}=1 \mathrm{~mm}$

1 inch $=254 \mathrm{~mm}=254.000 \mu \mathrm{m}$

Umrechnung der Auflösung von dpi in $\mu \mathrm{m}$

Auflösung $[\mu \mathrm{m}]=(2,54 /$ Auflösung [dpi] $) * 10.000$

$$
\text { z. B. } \quad \begin{aligned}
300 \mathrm{dpi} & =(2,54 / 300) * 10.000=84,67 \mu \mathrm{m} \\
600 \mathrm{dpi} & =(2,54 / 600) * 10.000=42,33 \mu \mathrm{m}
\end{aligned}
$$

Umrechnung der Auflösung von $\mu \mathrm{m}$ in dpi

Auflösung [dpi] $=(2,54 /$ Auflösung $[\mu \mathrm{m}]) * 10.000$

Umrechnung der Auflösung von $\mu \mathrm{m}$ in Linienpaare pro $\mathrm{mm}$

Auflösung $[\mathrm{lp} / \mathrm{mm}]=500 /$ Auflösung $[\mu \mathrm{m}]$ 
Umrechnung der Auflösung von Linienpaaren pro mm in $\mu \mathrm{m}$

Auflösung $[\mu \mathrm{m}]=500 /$ Auflösung $[\mathrm{lp} / \mathrm{mm}]$

Umrechnung der Auflösung von Linienpaaren pro $\mathrm{mm}$ in dpi

Auflösung [dpi] = 50,8*Auflösung $[\mathrm{lp} / \mathrm{mm}]$

Umrechnung der Auflösung von dpi in Linienpaare pro $\mathrm{mm}$

Auflösung $[\mathrm{lp} / \mathrm{mm}]=$ Auflösung $[\mathrm{dpi}] / 50,8$

\subsubsection{Scanauflösung}

Aufgrund der üblichen Korngröße von Luftbildfilmen erfordern analoge Luftbilder in der Regel keine höhere Scanauflösung als $10 \mu \mathrm{m}$. Die Auflösung von Farbfilmen (RGB, CIR) ist dabei häufig niedriger als die Auflösung panchromatischer Filme. Die folgende Tabelle 7 liefert Richtwerte für die maximale Auflösung beim Scannen.

Tabelle 7: $\quad$ Richtwerte zur Wabl der Scanauflösung in Abhängigkeit vom Photomaterial (GEOSYSTEMS 2001)

\begin{tabular}{l|c|c|c|c|c}
\hline $\begin{array}{l}\text { Analoges } \\
\text { Filmmaterial }\end{array}$ & $\begin{array}{c}\text { Auflösung } \\
(\mathrm{lp} / \mathrm{mm})\end{array}$ & $\begin{array}{c}\text { Filmnegativ/ Filmpositiv } \\
\text { Scan- } \\
\text { auflösung } \\
(\mu \mathrm{m})\end{array}$ & $\begin{array}{c}\text { Patei- } \\
\text { größe* } \\
(\mathrm{MB})\end{array}$ & $\begin{array}{c}\text { Papierabzug } \\
\text { Scan- } \\
\text { auflösung } \\
(\mu \mathrm{m})\end{array}$ & $\begin{array}{c}\text { Datei- } \\
\text { größe* } \\
(\mathrm{MB})\end{array}$ \\
\hline $\begin{array}{l}\text { Panchromatisch } \\
(\mathrm{SW})\end{array}$ & $320-50$ & 10 & 539 & 12,5 & 345 \\
$\begin{array}{l}\text { Farbe (RGB) } \\
\begin{array}{l}\text { Farbinfrarot } \\
\text { (CIR) }\end{array}\end{array}$ & $50-40$ & 12,5 & 1.032 & 15 & 716 \\
\hline
\end{tabular}

* radiometrische Auflösung 8 Bit

Für bestimmte Anwendungen kann es notwendig sein, die geometrische Auflösung analoger Luftbilder möglichst vollständig auszuschöpfen. Die hierfür erforderliche Scanauflösung kann durch die folgende Formel berechnet werden:

Scanauflösung $[\mu \mathrm{m}]=500 /$ Auflösungsvermögen des analogen Bildes $[\mathrm{lp} / \mathrm{mm}]$

Neben diesen theoretisch möglichen Auflösungen sollte bei der Wahl der Scanauflösung auch der Verwendungszweck der digitalisierten Bilder berücksichtigt werden. Dient das Luftbild als Kartengrundlage, kann eine niedrigere Scanauflösung gewählt werden. Für Luftbildanalysen sind höhere Auflösungen erforderlich. 
Die Differenzierbarkeit von Objekten und damit die Bodenauflösung werden von Bildmaßstab und Scanauflösung bestimmt. Tabelle 8 zeigt die Bodenauflösung in Metern in Abhängigkeit vom Bildmaßstab und der Scanauflösung. Wird eine bestimmte Bodenauflösung benötigt, kann diese hieraus abgeleitet werden. Beispiel: Es liegen Bilder im Maßstab 1:10.000 vor und es sollen $20 \mathrm{~cm}$ Bodenauflösung erreicht werden. Die erforderliche Scanauflösung beträgt $20 \mu \mathrm{m}$.

Tabelle 8: Bodenauflösung $[\mathrm{m}]$ in Abhängigkeit von Bildmaßstab und Scanauflösung

\begin{tabular}{c|ccccc}
\hline Bildmaßstab & \multicolumn{5}{|c}{ Scanauflösung } \\
& $10 \mu \mathrm{m}$ & $12,5 \mu \mathrm{m}$ & $15 \mu \mathrm{m}$ & $20 \mu \mathrm{m}$ & $40 \mu \mathrm{m}$ \\
\hline $1: 3.500$ & 0,035 & 0,044 & 0,052 & 0,070 & 0,140 \\
$1: 5.000$ & 0,050 & 0,065 & 0,075 & 0,100 & 0,200 \\
$1: 10.000$ & 0,100 & 0,125 & 0,150 & 0,200 & 0,400 \\
$1: 12.000$ & 0,120 & 0,150 & 0,180 & 0,240 & 0,480 \\
$1: 18.000$ & 0,180 & 0,225 & 0,270 & 0,360 & 0,720 \\
$1: 25.000$ & 0,250 & 0,313 & 0,375 & 0,500 & 1,000 \\
$1: 50.000$ & 0,500 & 0,625 & 0,750 & 1,000 & 2,000 \\
\hline
\end{tabular}

\subsubsection{Radiometrische Auflösung}

Beim Scannen werden die kontinuierlichen Farb- oder Grauwerte des analogen Luftbildes in diskrete Grauwertstufen umgewandelt. Die Speicherung erfolgt bei digitalen Schwarzweiß-Luftbildern in einem panchromatischen Kanal, bei digitalen Farbluftbildern in den drei Farbkanälen Rot, Grün und Blau.

Die Menge der verfügbaren Farbinformationen je Kanal wird in Bit pro Pixel angegeben. Bei einer Auflösung von $\mathrm{n}$ Bit können $2^{\mathrm{n}}$ verschiedene Farb- bzw. Grauwertstufen angegeben werden. Üblich sind Auflösungen von 8 Bit je Farbkanal, was 256 Grauwertstufen entspricht.

\subsubsection{Dateigrößen}

Die zu erwartenden Dateigrößen hängen von der Scan- bzw. der gewünschten Bodenauflösung ab (s. Tab. 9 und 10). Die Dateigröße von Farb- gegenüber SWBildern verdreifacht sich, da jeder Farbkanal (Rot, Grün, Blau) mit 8 oder 16 Bit abgespeichert wird. 
Tabelle 9: $\quad$ Dateigröße eines gescannten Luftbildes in Abbängigkeit von der Scanauflösung (GEOSYSTEMS 2001)

\begin{tabular}{cc|cc|cc}
\hline \multicolumn{2}{c|}{ Scanauflösung } & \multicolumn{2}{|c}{ Dateigröße SW-Bild } & \multicolumn{2}{c}{ Dateigröße Farbbild } \\
$\mu \mathrm{m}$ & $\mathrm{dpi}$ & $8 \mathrm{Bit}$ & $16 \mathrm{Bit}$ & $8 \mathrm{Bit}$ & $16 \mathrm{Bit}$ \\
\hline 5,0 & 5080 & $2.156,6$ & $4.313,2$ & $6.450,9$ & $12.939,7$ \\
10,0 & 2540 & 539,0 & $1.078,3$ & $1.612,7$ & $3.234,9$ \\
12,5 & 2032 & 345,0 & 690,1 & $1.032,1$ & $2.070,4$ \\
15,0 & 1693 & 239,5 & 479,0 & 716,5 & $1.437,1$ \\
20,0 & 1270 & 134,7 & 269,5 & 403,2 & 808,6 \\
25,0 & 1016 & 86,2 & 172,5 & 258,0 & 517,6 \\
30,0 & 847 & 59,9 & 120,0 & 179,3 & 359,7 \\
80,0 & 317 & 8,4 & 16,9 & 25,3 & 50,7 \\
\hline
\end{tabular}

Tabelle 10: Dateigröße je Quadratkilometer abgebildeter Fläche in Abbängigkeit von der Bodenauflösung

\begin{tabular}{c|cc|cc}
\hline \multirow{2}{*}{$\begin{array}{c}\text { Bodenauflösung } \\
(\mathrm{cm})\end{array}$} & \multicolumn{2}{|c|}{$\begin{array}{c}\text { Dateigröße SW-Bild } \\
(\mathrm{MB})\end{array}$} & \multicolumn{2}{c}{$\begin{array}{c}\text { Dateigröße Farbbild } \\
(\mathrm{MB})\end{array}$} \\
& $8 \mathrm{Bit}$ & $16 \mathrm{Bit}$ & $8 \mathrm{Bit}$ & $16 \mathrm{Bit}$ \\
\hline 100 & 1,0 & 1,9 & 2,9 & 5,7 \\
50 & 3,8 & 7,6 & 11,4 & 22,9 \\
40 & 6,0 & 11,9 & 17,9 & 35,8 \\
25 & 15,3 & 30,5 & 45,8 & 91,6 \\
20 & 23,8 & 47,7 & 71,5 & 143,1 \\
10 & 95,4 & 190,7 & 286,1 & 572,2 \\
\hline
\end{tabular}

Die zu erwartende Datenmenge kann auch in Abhängigkeit von der Bodenauflösung und der abgedeckten Fläche hergeleitet werden (s. Tab. 10). Die dortigen Angaben beziehen sich auf eine einfache Abdeckung. Überdeckungsbereiche für Stereoauswertungen müssen entsprechend ihrer Prozentangaben zugeschlagen werden. 


\subsection{Bildbearbeitung beim Scannen}

Digitale Bilder können schon beim Scannen auf eine spätere Fragestellung hin optimiert werden. Durch Veränderung der Radiometrie können Helligkeit, Bildkontraste und Farbbalance verbessert werden. Das Histogramm des digitalen Luftbildes sollte keine Lücken aufweisen und den vollen Grauwertbereich umfassen. Die Differenzierung bestimmter Gebiete (z. B. Wald) kann durch Streckung bestimmter Histogrammbereiche erhöht werden. Wichtig ist, die beim Scannen vorgenommenen Einstellungen zu dokumentieren. Bei all diesen Manipulationen ist zu beachten, dass Informationen des Originalbildes verloren gehen können. Bei Vergabe eines Scanauftrages ist es deshalb sinnvoll, mit dem Auftragnehmer die Einstellungen der Scanparameter im Hinblick auf den späteren Verwendungszweck zu besprechen. Empfehlenswert ist die Abstimmung über Probescans und die Lieferung von Sollbeispielen für die Farbbalance.

Das digitalisierte analoge Luftbild erfährt einen Informationsverlust gegenüber dem Original. Insbesondere in Schattenbereichen bietet es weniger Informationen, so dass hier eine Objekterfassung erschwert und die Bodensicht verschlechtert wird.

\subsection{Kosten für Luftbildscans}

Absolute Werte für die Kosten von Luftbildscans können an dieser Stelle nicht angegeben werden. Sie variieren stark in Abhängigkeit vom Ausgangsmaterial und der gewünschten Auflösung. Allerdings kann gesagt werden, dass das Scannen von der Luftbildrolle erheblich günstiger ist als Scans von Einzelbildern. 


\section{Planung, Vergabe und Durchführung digitaler Bildflüge}

Vor der Planung, Vergabe oder Durchführung eines digitalen Bildfluges sollten nachfolgend aufgeführte Kriterien beachtet und gegebenenfalls angepasst werden.

\subsection{Technische Rahmenbedingungen}

- Kamera: Es können digitale Hochleistungskameras mit GNSS/ INS oder low-cost Kameras eingesetzt werden (s. Kap. 2).

- Kanalkombination: Bei der digitalen Bildaufnahme werden in der Regel gleichzeitig vier Farbkanäle (R, G, B, NIR) und ein panchromatischer aufgenommen. Daraus abgeleitete, gängige Bildprodukte sind panchromatische Bilder, Vierkanal- (RGBNIR), Echtfarb- (RGB) und Farbinfrarot- bzw. CIR-Bilder (NIR-RG).

- Bildorientierung: Bei Auftragsvergabe muss festgelegt werden, ob die Daten der äußeren Orientierung (x, y, $z, \omega, \varphi$ und $x$, s. auch Abb. 6) vom Auftragnehmer bereitzustellen sind oder nicht. Die Orientierungsdaten können durch GNSS-/ INS-Aufzeichnungen oder durch Aerotriangulation bestimmt werden (s. Kap. 5.2). Sollen die Orientierungsdaten für die auszuliefernden Bilder vom Auftragnehmer bereitgestellt werden, so wird eine Genauigkeit bei $x$, y und $z$ von mindestens $50 \mathrm{~cm}$ und bei $\omega, \varphi$ und $x$ von 0,5 gon empfohlen.

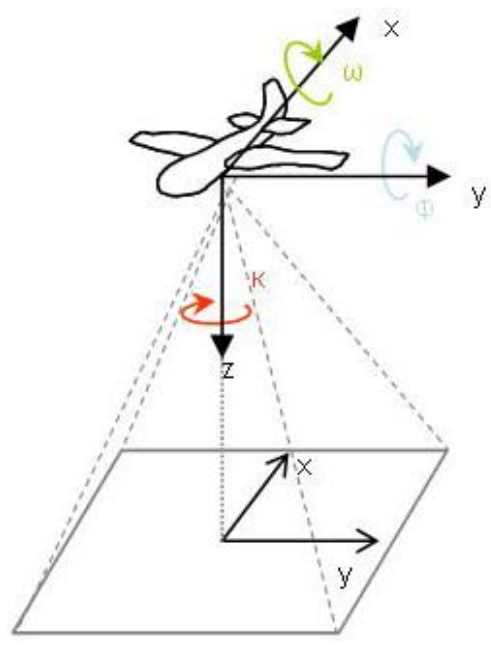

Abbildung 6: Parameter der äußeren Orientierung 
- Referenæsystem: Das horizontale und vertikale Referenzsystem sollte verbindlich und eindeutig festgelegt werden.

- Radiometrischer Abgleich: Die Bilder eines Projektes sollten untereinander eine ähnliche Radiometrie aufweisen. Dies erfordert eine dem Verwendungszweck entsprechende Angleichung im Zuge der Prozessierung durch die Befliegungsfirma (z. B. auf Wald oder landwirtschaftliche Flächen). Angleichungen erfolgen firmenspezifisch und derzeit ohne Transparenz für den Auftraggeber. Alternativ können Rohdaten ohne radiometrischen Abgleich bezogen werden.

\section{2 Äußere Rahmenbedingungen}

- Zeitpunkt der Befliegung: Der Befliegungszweck entscheidet über den Befliegungszeitraum. Dabei ist zu bedenken, dass bei niedrigen Sonnenständen unter $30^{\circ}$ über dem Horizont mit deutlich größeren Schattenbereichen zu rechnen ist.

- Bewölkung: Eine gleichmäßige, geschlossene Bewölkung ist dann zu akzeptieren, wenn dadurch die Bildqualität nicht beeinträchtigt wird und Wolkenschatten ausgeschlossen sind.

- Eventuelle Beeinträchtigungen durch Nebel, Dunst, Hochwasser, Schnee oder Eis müssen berücksichtigt werden.

\subsection{Flugplanung}

- Flugrichtung: In Abhängigkeit von der Richtung der Sonneneinstrahlung kommt es je nach Flugrichtung zu Helligkeitsunterschieden in den Bildaufnahmen. Dies kann durch digitale Nachbearbeitung weitgehend ausgeglichen werden. Die Flugrichtung kann daher frei z. B. nach der Form und Ausrichtung des Untersuchungsgebiets gewählt werden (Kostenoptimierung).

- Überdeckung: Die Bildaufnahme sollte so erfolgen, dass Stereoauswertungen möglich sind. Bei der Verwendung von Matrixkameras sollte die Überdeckung der Bilder in Längs- und Querrichtung wenigstens 60 bzw. 30 \% betragen (s. Abb. 7). Für die Erstellung von Oberflächenmodellen und Orthophotos in Gebirgslagen sollte die Überdeckung mindestens 70 bzw. $40 \%$ betragen. Bei Zeilenkameras wird keine Längsüberdeckung angegeben. Die Querüberdeckung sollte hier wenigstens $30 \%$ betragen.

- Flughöhenabweichung: In ebenem Gelände sollte sie max. $\pm 2 \%$ der Flughöhe über Grund zwischen zwei benachbarten Bildern betragen (s. Abb. 8). 


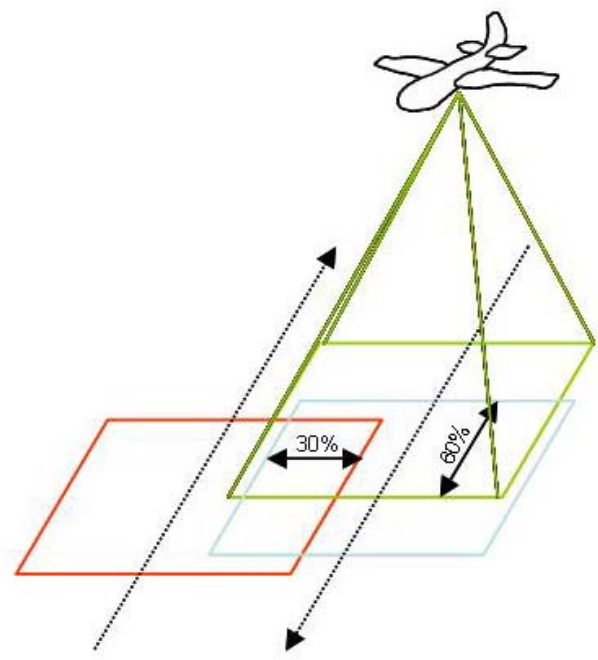

Flugstreifen $2 \quad$ Flugstreifen 1

Abbildung 7: Längsüberdeckung zwischen Bildern eines Flugstreifens und Querüberdeckung zwischen benachbarten Flugstreifen

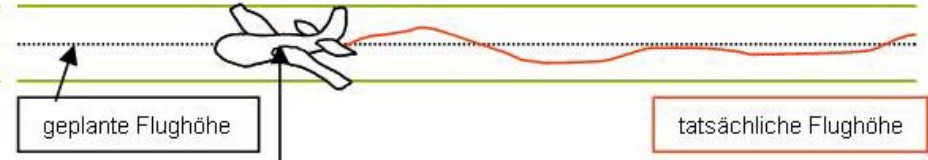

Flughöhe über Grund

ebenes Gelände

\section{Abbildung 8: Flughöhenabweichung}

- Abweichung der Drehwinkel: Die Bildneigung $\varphi$ (Nicken) soll max. $\pm 2,0$ gon und $\omega$ (Rollen) max. \pm 4 gon sowie die Bildkantung $x$ (Gieren) nicht mehr als \pm 5,0 gon zur Flugachse betragen. Größere Abweichungen verursachen störende Verzerrungen im Bild.

- Zustimmung zur Bildflugplanung: Die Flugplanung der Befliegungsfirma bedarf der Freigabe durch den Auftraggeber. Zu diesem Zweck sind die geplanten Bildmitten und Streifen in digitaler Form (z. B. ASCII- oder Shapefile) oder als Karte vorzulegen.

- Fluginformation: Der Auftragnehmer hat den Auftraggeber über Beginn und/oder Ende eines Flugtages in Kenntnis zu setzen. 
- Bodenauflösung: Die nötige Bodenauflösung (s. Kap. 2.3.3) ist im Hinblick auf die Fragestellung und die zur Verfügung stehende technische Ausstattung, z. B. Speicherkapazität, zu bestimmen (s. Kap. 9).

\subsection{Datenübergabe}

- Fluggrotokoll sollte enthalten: Auflistung der Flugstreifen- und Bildnummern, Flugtag, Aufnahmezeit, Flughöhe über Grund, horizontale Sichtweite, Bewölkung, Bodenauflösung, Längs-/Querüberdeckung, verwendete Kamera, Brennweite, Blende, Belichtungszeit, verwendete Filter, Erläuterungen von Besonderheiten und Abweichungen.

- Kalibrierungsprotokolle der verwendeten Kamera inkl. Angaben über die Spektralbereiche der Farbkanäle.

- Bildmittenübersicht/Bildmittenkoordinaten digital und/oder analog.

- Benennung der gelieferten Bildprodukte mit ihrer jeweiligen Bildkanalbelegung.

- Datenformat der Bilddateien: abgestimmt auf Auswertesoftware; als Standardausgabeformat hat sich Untiled TIFF, unkomprimiert mit 8 bzw. 16 Bit Farbtiefe bewährt.

- Datenbereitstellung: mobile Festplatte, DVD, Magnetband oder FTP (File Transfer Protocol).

- Referenæsystem: Das horizontale und vertikale Referenzsystem sollte eindeutig dokumentiert sein.

- Bezeichnung der Bilddateien: Die Bezeichnung der Bilder sollte systematisch und eindeutig für eine spätere Archivierung sein, z. B. eine Kombination aus Flugstreifen und Bildnummer, Jahr der Befliegung, Gebietsbezeichnung, Kurzbezeichnung für Bildfarbformat (RGBNIR, RGB, CIR, PAN), radiometrische Auflösung (8 Bit/16 Bit).

- Äßßere Orientierungsparameter müssen in einer eindeutig definierten Datenstruktur abgelegt sein und sollten Angaben über die Art ihrer Bestimmung (direkte oder indirekte Georeferenzierung), das verwendete Referenzsystem und die Maßeinheit der Drehwinkel enthalten. Eine ASCII-Datei kann z. B. wie folgt aufgebaut sein: Streifennummer / Bildnummer / Aufnahmezeit / Rechtswert / Hochwert / Flughöhe ü. NN / Drehwinkel $\omega, \varphi$, und $x$.

- Liefertermine sollten in Abhängigkeit vom Abschluss der Befliegung vereinbart werden (üblicherweise 2-4 Wochen).

- Nutzungsrecht: Der Auftraggeber sollte das Nutzungsrecht an den angefertigten Bilddaten schriftlich festlegen (z. B. alleiniges Nutzungsrecht für den Auftraggeber, zusätzliches Nutzungsrecht für den Auftragnehmer, Nachnutzungsrechte für den Auftragnehmer zur Ableitung von Folgeprodukten). 
Die oben beschriebenen Punkte sollten in einem Übergabeprotokoll dokumentiert werden (s. Anhang), das gleichzeitig der Generierung von Metadaten dient.

\subsection{Kosten einer Luftbildbefliegung}

Die Kosten einer Luftbildbefliegung werden v. a. durch nachstehende Faktoren beeinflusst:

- Gebietsgröße und -ausformung bzw. Anzahl der erforderlichen Flugstreifen

- Lage des Aufnahmegebietes (Anflugentfernung)

- Überdeckungsgrad der Luftbilder, insbesondere Querüberdeckung

- Art der Kamera

- Bodenauflösung

- Genauigkeitsanforderungen bei der Bildorientierung

- Gestaltung der Urheber- und Nutzungsrechte 



\section{Bildorientierung}

Unter Bildorientierung wird die Transformation von Bildkoordinaten in ein übergeordnetes Koordinatensystem (z. B. Landeskoordinatensystem) verstanden.

Sie ist eine wesentliche Voraussetzung für die lagegenaue Auswertung, die Verbindung mit anderen Geodaten sowie die Herstellung von Orthophotos und Höhenmodellen. Die Bildorientierung gliedert sich in die innere und die äußere Orientierung.

\subsection{Innere Orientierung}

Die innere Orientierung beschreibt die Geometrie der Kamera durch die Parameter Brennweite, Koordinaten des Bildhauptpunktes, Linsenverzerrung und Bildgröße, welche mit Hilfe von Kalibrierungsverfahren bestimmt werden.

Die Größe und Lage des Bildes wird bei digitalisierten, analogen Bildern durch die Koordinaten der Rahmenmarken beschrieben.

Bei Matrixkameras wird die Bildausdehnung durch die Sensorgröße definiert (Pixelgröße, Pixelanzahl in x- und y-Richtung).

Bei Zeilenkameras ist die Bildausdehnung ebenfalls durch die Sensorgröße definiert. Hier besteht der Sensor allerdings nur aus einer Pixelzeile (z. B. ADS80 die Größe des Sensors beträgt 12.000 x 1 Pixel). Erst durch das Aneinanderfügen einzelner Bildzeilen entsteht der Bildstreifen.

Die Parameter der inneren Orientierung kann man dem Kalibrierungsprotokoll entnehmen. Dieses muss von der Befliegungsfirma angefordert werden.

\section{2 Äußere Orientierung}

Die äußere Orientierung gibt die Lage der Bildebene zum Aufnahmezeitpunkt an, die definiert wird durch die $\mathrm{x}$-, $\mathrm{y}$ - und z-Koordinaten des Projektionszentrums sowie die drei Drehwinkel $\omega, \varphi$ und $x$. Diese Werte können durch GNSS- und INSAufzeichnungen oder durch Aerotriangulation bestimmt werden.

In Abhängigkeit von der Genauigkeit der GNSS-/INS-Daten und der Flughöhe können Objekte bereits mit einer Lagegenauigkeit von $0,5 \mathrm{~m}$ bis $5 \mathrm{~m}$ gemessen werden. Sollen höhere Genauigkeiten erreicht werden, muss eine Aerotriangulation durchgeführt werden. Die benötigten Passpunkte können selbst erhoben oder zum Beispiel von den Landesvermessungsämtern bezogen werden. 


\subsection{Aerotriangulation}

Die Aerotriangulation ist eine Methode, mit der in einem Schritt simultan die äußere Orientierung auch großer Bildblöcke bestimmt wird. Als Eingangsparameter dienen Verknüpfungspunkte, Passpunkte und näherungsweise Projektionszentren.

Die Genauigkeit wird im Wesentlichen beeinflusst von:

- der Bodenauflösung bzw. dem Bildmaßstab

- der Art und Genauigkeit der Passpunkte

- der Anzahl der Passpunkte

- der Verteilung der Passpunkte im Bildblock

- der Bildqualität (z. B. Bildkontrast, Bildrauschen)

Passpunkte sollten möglichst an den Blockrändern und in den Blockecken gesetzt werden.

Die absolute Genauigkeit einer Aerotriangulation kann durch Kontrollmessungen von bekannten Punkten in einem orientierten Bildblock ermittelt werden (Kontrollpunkte mit bekannten Koordinaten). Diese Punkte dürfen vorher nicht in die Ausgleichungsrechnung mit eingegangen sein.

Bei der Auswertung von Zeitreihen ist es wichtig, die Lageverschiebungen zwischen den Bildjahrgängen möglichst gering zu halten. Hierfür bietet sich an, die Passpunkte aus einem bereits orientierten Vergleichsbildflug zu übertragen. 


\section{Orthophotoerstellung}

Luftbilder geben die Landschaft verzerrt wieder. Sie haben im Gegensatz zu Karten den Nachteil, dass es nicht möglich ist, Distanzen, Flächen und Geometrien zu messen. Orthophotos hingegen bieten dem Nutzer eine geometrisch korrekte, kartengleiche Abbildung der Landschaft (s. Abb. 9).
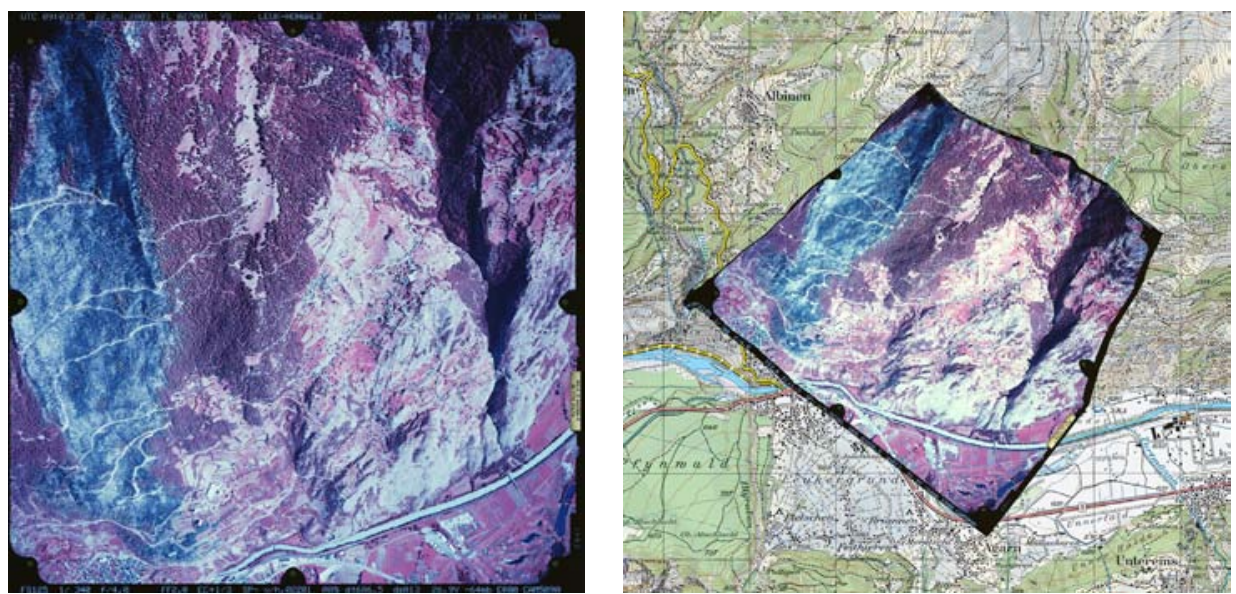

Abbildung 9: Auswirkung der Orthorektifizierung: verzerrtes Original-Luftbild (links), entzerrtes Orthophoto (rechts). Quelle: Pixelkarte (C) 2011 swisstopo (5704 000 000), Luftbild (C) $B A F U / W S L$

Mit den Orientierungsdaten der Luftbilder und einem digitalen Höhenmodell können Orthophotos berechnet werden (s. Abb. 10). Dabei werden Verzerrungen, die bei der Aufnahme eines Bildes durch die Zentralprojektion und unterschiedliche Geländehöhen entstehen, rechnerisch weitgehend eliminiert. Das Ergebnis ist ein digitales Orthophoto (DOP), ein maßstäbliches Luftbild mit kartengleicher Parallelprojektion.

Zur Orthophotoberechnung können zwei unterschiedliche Arten von Höhenmodellen alternativ herangezogen werden:

1. Das Digitale Geländemodell (DGM) oder Digital Terrain Model (DTM) beschreibt die Höhe des Erdbodens (Gelände) ohne Gebäude oder Vegetation.

2. Das Digitale Oberflächenmodell (DOM) oder Digital Surface Model (DSM) beschreibt die Höhe der Erdoberfläche einschließlich aller natürlichen und künstlichen Objekte wie z. B. Vegetation und Gebäude. 


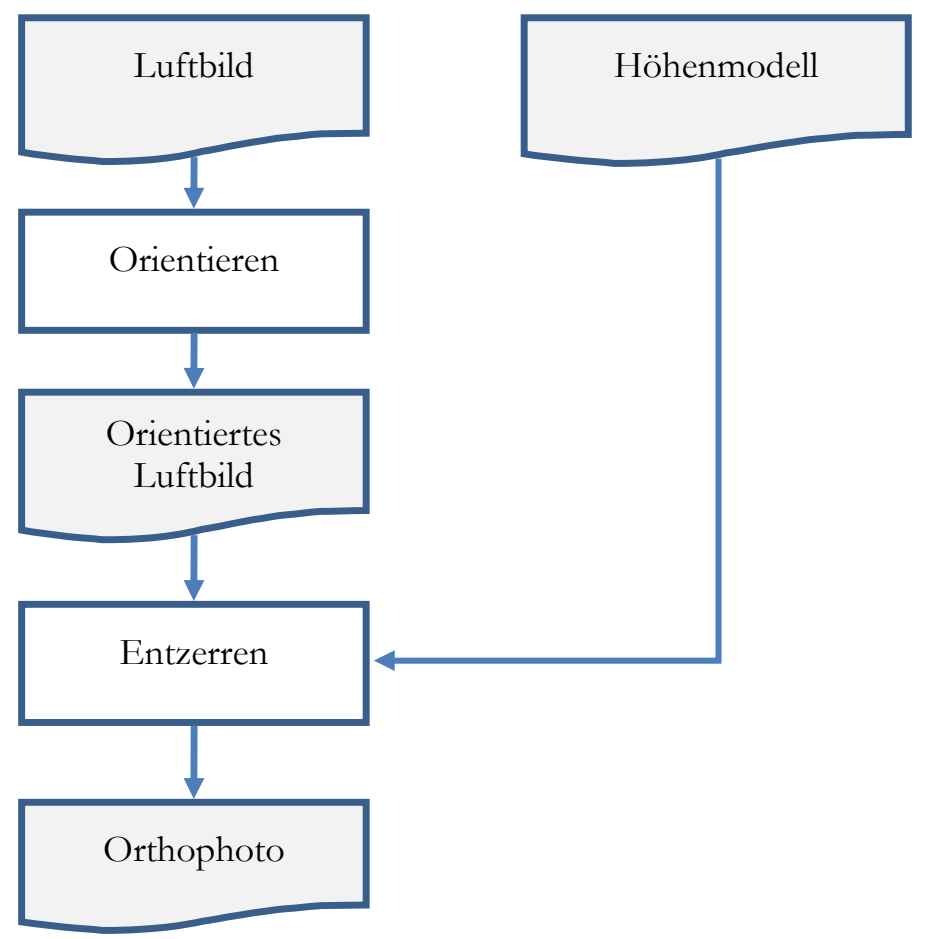

Abbildung 10: Ablaufschema der Orthophotoerstellung

Die Genauigkeit von Orthophotos hängt außer von den Ergebnissen der Bildorientierung und Aerotriangulation vor allem von dem verwendeten Höhenmodell ab. Denn nur tatsächlich auf der Oberfläche des Höhenmodells liegende Bildpunkte werden korrekt entzerrt und lagerichtig wiedergegeben. Objekte, die sich über oder unter dieser Oberfläche befinden, weisen einen Lagefehler auf. Die Auswirkung dieser Lagefehler zeigt ein Beispiel in Abbildung 11. Dort werden Orthophotos gezeigt, die aus benachbarten Luftbildern erstellt wurden. Für die Entzerrung wurde ein Digitales Geländemodell verwendet. Sämtliche Objekte über der Geländehöhe, wie z. B. Bäume oder Strommasten, werden verkippt abgebildet (im linken Bild nach rechts, im rechten Bild nach links). Der grüne Kreis markiert in den Bildern die gleiche Position, es werden aber unterschiedliche Baumkronen an der Position dargestellt.

Da die beschriebenen Lagefehler zum Bildrand der Ausgangsbilder hin zunehmen (Kippeffekte), sollten beim Mosaikieren von Orthophotos möglichst nur zentrale Bildteile der originalen Luftbilder verwendet werden. 

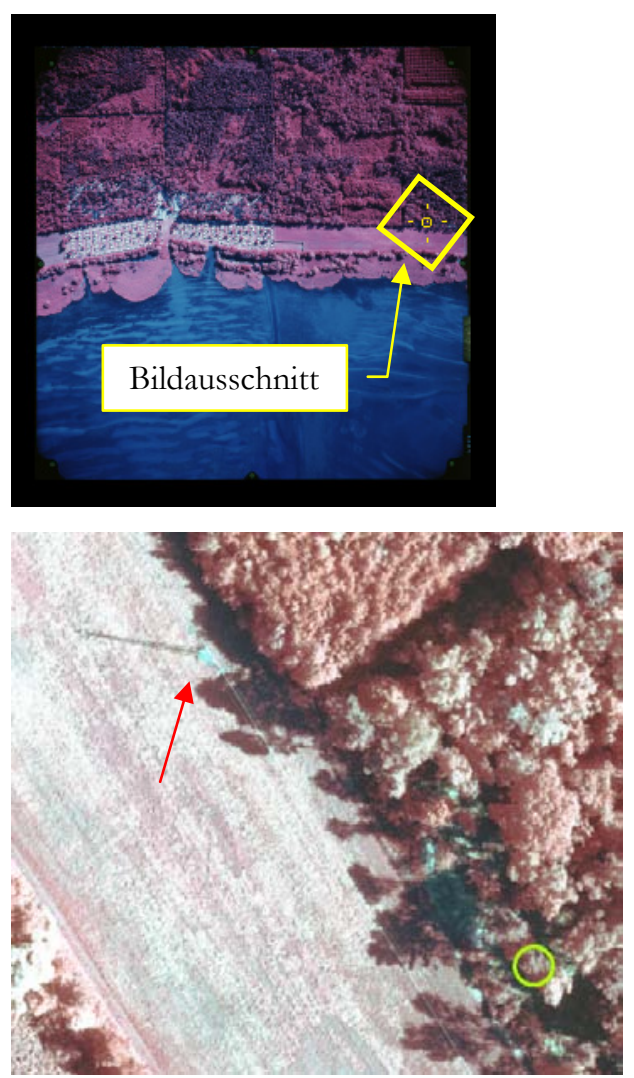
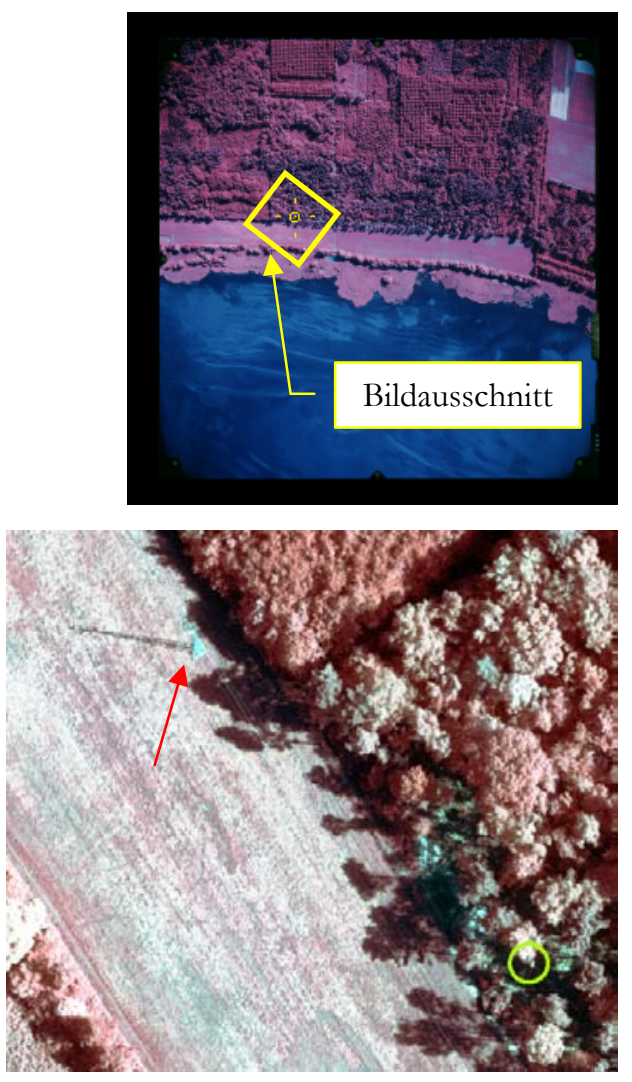

Abbildung 11: Orthophotos, erstellt mit einem Geländemodell aus benachbarten Luftbildern (mit Objek.tverschiebung)

So wird zum Beispiel in einem mit einer digitalen Kamera aufgenommenen Luftbild (UltraCamXp mit $\mathrm{c}=100,5 \mathrm{~mm}$ ) die Krone eines 25 Meter hohen Baumes am Bildrand ( $5 \mathrm{~cm}$ vom Nadir entfernt) gemäß nachstehender Formel um ca. 12 Meter versetzt abgebildet.

$\Delta r_{0}=\frac{\mathrm{r}^{\prime}}{\mathrm{c}} \times \Delta h$

$\Delta r_{0}=$ radialer Objektversatz

$\mathrm{r}^{\prime} \quad=$ Abstand des Bodenpunktes des Objektes vom Nadir

c $=$ Kammerkonstante

$\Delta \mathrm{h}=$ Objekthöhe

Beiträge aus der NW-FVA, Band 7, 2012 
Unerwünschte Effekte dieser Verkippungen sind verdeckte Bildbereiche sowie falsche Baumkronen in Probekreisen, deren Lage auf die Geländehöhe bezogen wird (s. Kap. 7.1) Dieses kann durch die Benutzung eines digitalen Oberflächenmodells bei der Erstellung von Orthophotos vermieden werden. Bei diesem Vorgehen werden sämtliche Objekte über der Geländehöhe nicht verkippt, sondern ,aufgerichtet“ dargestellt. Abbildung 12 zeigt Orthophotos, die mit einem Digitalen Oberflächenmodell entzerrt wurden. Im grünen Kreis wird jetzt die identische Baumkrone lagerichtig abgebildet.
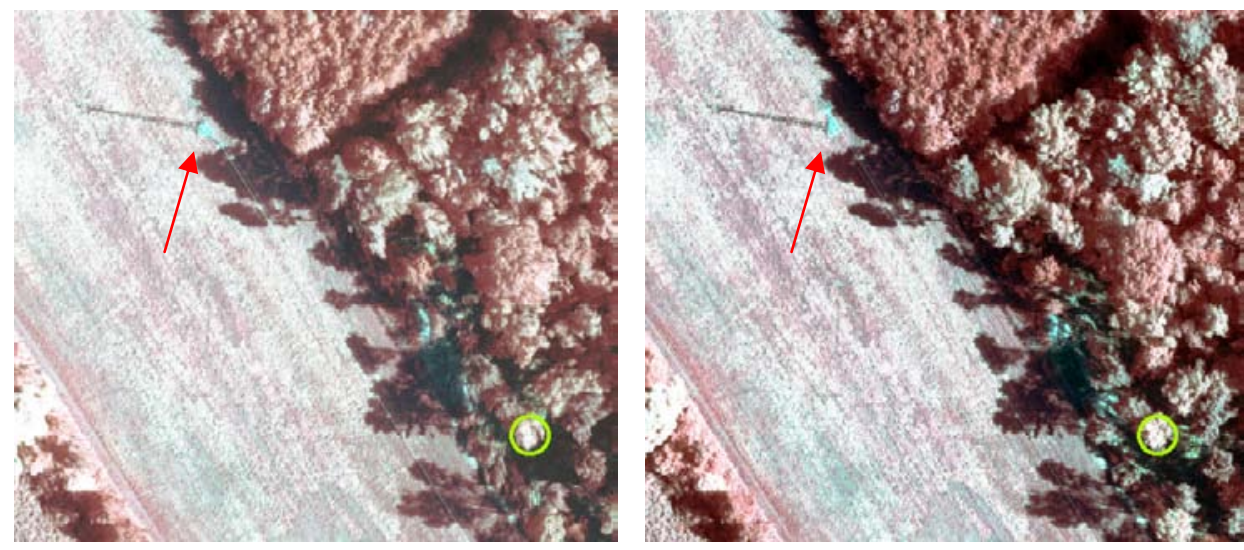

Abbildung 12: Orthophotos, erstellt mit einem Oberflächenmodell aus benachbarten Luftbildern (ohne Objektverschiebung)

Bei diesem Vorgehen ist zu beachten, dass hinter hohen Objekten (z. B. Bäumen) wegen der rechnerischen Entzerrung Bereiche im Orthophoto entstehen, für die im originalen Luftbild keine Information vorhanden ist. Diese sichttoten Bereiche werden durch Interpolation benachbarter Pixel mit Bildinformation gefüllt, wodurch Artefakte entstehen können (s. Abb. 13).

Das linke Orthophoto wurde mit einem Geländemodell entzerrt. Die Kronenstruktur ist gut abgebildet. Das rechte Orthophoto wurde mit einem Oberflächenmodell entzerrt. Durch das „Aufrichten“ des Baumes bei der Rektifizierung sind Artefakte entstanden. Beim Erstellen eines Orthophotos muss daher immer ein Kompromiss zwischen Lagegenauigkeit und dem Verlust von Bildtextur eingegangen werden.

Höhenmodelle können aus unterschiedlichen Daten gewonnen werden. Die folgende Tabelle 11 beschreibt Vor- und Nachteile der jeweiligen Produkte. Alle Angaben für das DOM gelten prinzipiell auch für das DGM. Beim DOM ist zu beachten, dass bei Winteraufnahmen von Laubbäumen unabhängig vom Sensor mit Qualitätseinbußen zu rechnen ist. 

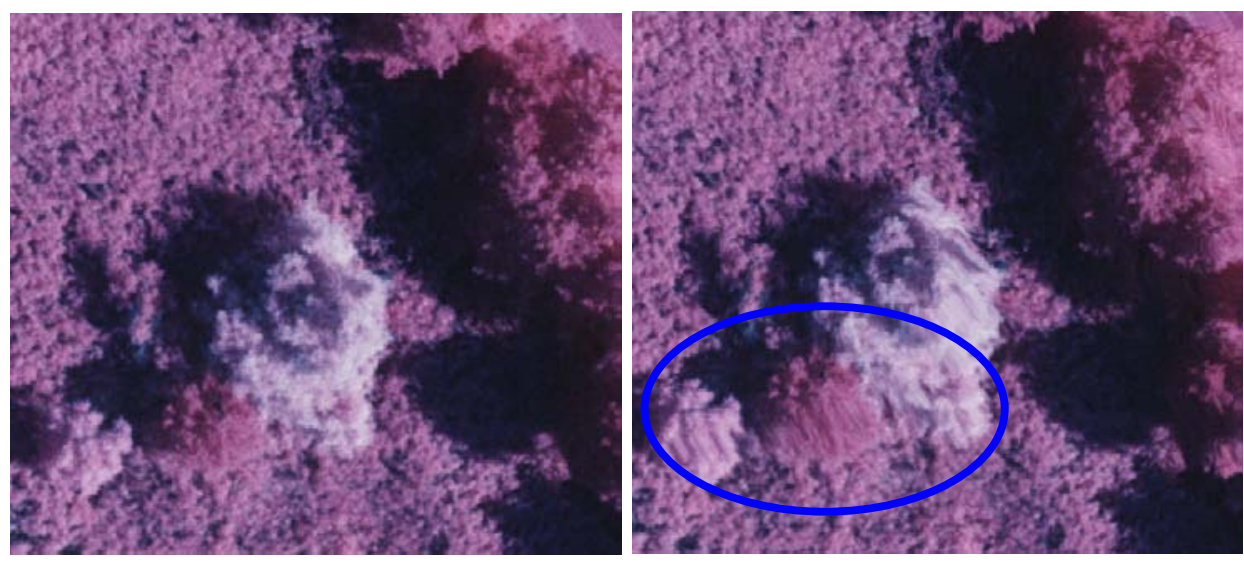

Abbildung 13: Artefaktbildung bei Verwendung eines DOM - links: Entzerrung mit DGM, rechts: Entzerrung mit DOM

Großflächige Höhenmodelle werden meistens durch Fachfirmen oder Fachverwaltungen erstellt und sollten grundsätzlich vor Benutzung auf ihre Qualität überprüft werden. Erste Informationen sind den mitzuliefernden Metadaten (s. Kap. 8.3.3) zu entnehmen.

In Deutschland, Österreich und der Schweiz sind flächendeckende Höhenmodelle - die je nach Bundesland/Kanton und teilweise innerhalb der Bundesländer in der Qualität und im Erstellungsverfahren variieren können - bei den nachfolgenden Institutionen vorhanden und zu beziehen.

\section{Deutschland:}

Bundesamt für Kartographie und Geodäsie (http://www.geodatenzentrum.de/)

- DGM25: Flächendeckend verfügbar. Gitterweite 25 m

Höhengenauigkeit +/- 1-5 m

Vermessungsverwaltungen der Bundesländer

- Landesweite Geländemodelle mit Gitterweiten von 1-25 m in unterschiedlicher Qualität und Verfügbarkeit

\section{Schweiz:}

Bundesamt für Landestopografie (http://www.swisstopo.ch/)

- DHM25: Flächendeckend verfügbar. Gitterweite $25 \mathrm{~m}$ Höhengenauigkeit +/- 1,5-3 $\mathrm{m} \sim 0,33$ Euro $/ \mathrm{km}^{2}$

- swissALTI3D: Flächendeckend verfügbar bis $2.000 \mathrm{~m}$ ü. NN (Stand 07/2011). Gitterweite $2 \mathrm{~m}$ Höhengenauigkeit +/- 0,5 m

Vermessungsämter der Kantone

- Kantonsweite Geländemodelle in unterschiedlicher Qualität und Verfügbarkeit 
Tabelle 11: Herkunft und Eigenschaften digitaler Höhenmodelle

\begin{tabular}{|c|c|c|}
\hline Herkunft & Vorteile & Nachteile \\
\hline $\begin{array}{l}\text { aus Laserscanner- } \\
\text { Befliegungen (LIDAR) }\end{array}$ & $\begin{array}{l}\text { + sehr genaue Höhenermittlung } \\
+ \text { sehr gute Abbildung der Mor- } \\
\text { phologie der Oberfläche bei } \\
\text { hoher Punktdichte } \\
+ \text { auch in Vegetationsbeständen } \\
\text { kann das Gelände erfasst werden } \\
\text { in Abhängigkeit von der Vegeta- } \\
\text { tionsart, Vegetationsdichte und } \\
\text { Befliegungszeitpunkt }\end{array}$ & $\begin{array}{l}\text { - kostenintensiv } \\
\text { - große Datenmengen }\end{array}$ \\
\hline $\begin{array}{l}\text { photogrammetrisch } \\
\text { manuell aus Luftbild- } \\
\text { Stereomodellen }\end{array}$ & $\begin{aligned}+ & \text { sehr genau }(0,2 \% \text { der Flughöhe } \\
& \text { über Grund) } \\
+ & \text { Bruchkanten erfassbar } \\
+ & \text { frei wählbare Punktverteilung } \\
+ & \text { zeitgleich kombinierbar mit } \\
& \text { Interpretationsarbeiten }\end{aligned}$ & $\begin{array}{l}\text { - von Bodensicht abhängige } \\
\text { Punkteverteilung } \\
\text { - sehr hoher Messaufwand, } \\
\text { deswegen nur für kleine } \\
\text { Flächen geeignet (Stichproben) } \\
\text { - kostenintensiv }\end{array}$ \\
\hline $\begin{array}{l}\text { photogrammetrisch } \\
\text { automatisch aus } \\
\text { Luftbild- } \\
\text { Stereomodellen }\end{array}$ & $\begin{aligned}+ & \text { sehr genau }(0,2 \% \text { der Flughöhe } \\
& \text { über Grund) } \\
+ & \text { große Flächen bearbeitbar } \\
+ & \text { sehr gute Abbildung der Mor- } \\
& \text { phologie der Oberfläche bei } \\
& \text { hoher Punktdichte } \\
+ & \text { DOM korreliert sehr gut mit den } \\
& \text { Bildinhalten der Luftbilder }\end{aligned}$ & $\begin{array}{l}\text { - bei digitalisierten Luftbildern } \\
\text { problematisch in Schattenbe- } \\
\text { reichen } \\
\text { - problematisch bei geringem } \\
\text { Kontrastunterschied (z. B. } \\
\text { See) oder regelmäßigen } \\
\text { Strukturen (z. B. Solaranlage) } \\
\text { - sehr rechenintensiv } \\
\text { - große Datenmengen }\end{array}$ \\
\hline Radar & $\begin{array}{l}\text { + Oberflächenmodell nahezu } \\
\text { weltweit vorhanden, Space } \\
\text { shuttle mission (SRTM) } \\
+ \text { kostenlos } \\
\text { (zusätzlich nutzbar: TerraSAR-X } \\
\text { TanDEM-X mit einer Bodenauf- } \\
\text { lösung von } 12 \mathrm{~m} \text { und Höhen- } \\
\text { genauigkeit } \leq 2 \mathrm{~m} \text { ) }\end{array}$ & $\begin{array}{l}\text { - grobe Abbildung der Ober- } \\
\text { fläche aufgrund Rasterweite } \\
\text { von } 90 \mathrm{~m} \\
\text { - im alpinen Bereich } \\
\text { unvollständig und ungenau }\end{array}$ \\
\hline optische Satelliten & $\begin{array}{l}\text { + weltweit vorhanden, aus } \\
\text { ASTER- Satellitenbildern } \\
+ \text { kostenlos } \\
\text { (auch aus Stereo-Satellitenbilder } \\
\text { der neuen Generation möglich, } \\
\text { jedoch kostenintensiv; Aussagen } \\
\text { zur Genauigkeit derzeit nicht } \\
\text { möglich) }\end{array}$ & $\begin{array}{l}\text { - grobe Abbildung der Ober- } \\
\text { fläche aufgrund der } \\
\text { Rasterweite von } 30 \mathrm{~m}, \\
\text { Höhengenauigkeit } 10-25 \mathrm{~m}\end{array}$ \\
\hline $\begin{array}{l}\text { terrestrische } \\
\text { Messungen }\end{array}$ & sehr genau & $\begin{array}{l}\text { - sehr hoher Aufwand, daher } \\
\text { auf Sonderanwendungen (z. B. } \\
\text { Renaturierung) begrenzt }\end{array}$ \\
\hline
\end{tabular}




\section{Österreich:}

Bundesamt für Eich- und Vermesssungswesen (http://www.bev.gv.at)

- DGM mit Gitterweite ab 10 m flächendeckend verfügbar ( 3,0 Euro/ $\left.\mathrm{km}^{2}\right)$

GIS-Abteilungen der Bundesländer

- Digitale Geländemodelle mit einer Gitterweite von $1 \mathrm{~m}$ aus LaserscannerBefliegungen, verfügbar nach Stand der Befliegungen; Informationen unter http://www.geoland.at/

Welt:

Zusätzlich sind weltweite Oberflächenmodelle mit geringerer Qualität unter folgenden Adressen kostenlos verfügbar:

- SRTM mit 90 m Bodenauflösung: http://www.glcf.umd.edu/data/srtm/; http://srtm.csi.cgiar.org/

- ASTER mit 30 m Bodenauflösung: http://www.gdem.aster.ersdac.or.jp 



\section{Auswertung digitaler Luftbilder}

Die Auswertung von Luftbildern erfolgt immer zweckgebunden. Um die gewünschten Ziele zu erreichen, ist im Vorfeld ein intensiver Austausch über Definitionen (z. B. Walddefinition) und Nomenklatur (z. B. Altersklassen, Waldentwicklungsstufen, s. auch ARBEITSGRUPPE FORSTLICHER LUFTBILDINTERPRETEN 1998, 1999 u. 2003) zwischen den Akteuren notwendig.

Die digitale Bildauswertung bietet gegenüber der Arbeit mit analogen Bildern Vorteile. Digitale Luftbilder können durch Bildbearbeitungsschritte für die jeweilige Auswertung optimiert werden (s. Kap. 2 und 3). Während des Auswertungsvorganges ist ein schneller Bild- und Modellwechsel möglich. Kopierte Bilddaten können von mehreren Betrachtern gleichzeitig an verschiedenen Orten ausgewertet werden. Orientierte Bilddaten und daraus abgeleitete Auswertungsergebnisse können jederzeit mit bereits vorliegenden Geobasisdaten und Geofachdaten in einer GIS-Umgebung dargestellt und analysiert werden. Dies ist besonders für Monitoringaufgaben vorteilhaft.

Digitale Luftbilder eröffnen die Möglichkeit des Einsatzes automatisierter oder teilautomatisierter Arbeitsschritte.

Es wird an dieser Stelle nochmals darauf hingewiesen, dass digitalisierte analoge Luftbilder nicht die Qualität eines digitalen Luftbildes erreichen. Im Folgenden wird auf die Besonderheiten der Auswertung von digitalen Stereoluftbildern und Orthophotos eingegangen.

\subsection{Die visuelle Auswertung digitaler Orthophotos}

Die thematische und kartographische Auswertung digitaler Orthophotos findet in der Regel in einer GIS-Umgebung statt. Damit stehen umfangreiche Werkzeuge zur Visualisierung, Digitalisierung und Verknüpfung mit anderen Geodaten zur Verfügung.

Die fehlende Höheninformation erlaubt nur eingeschränkte Auswertungen von vertikalen und horizontalen Bestandes- bzw. Strukturmerkmalen. Der Informationsgehalt von Luftbildern wird bei Orthophotos nicht voll ausgeschöpft. Dies gilt insbesondere in Schattenbereichen. Bei der Orthophotoherstellung kann es zu Farbveränderungen kommen, die das Ergebnis der Interpretation beeinflussen.

Bei der Digitalisierung von Objekten liegen die Ergebnisse unmittelbar georeferenziert vor. Die Lagegenauigkeit hängt hierbei von der geometrischen Qualität der verwendeten Orthophotos ab (vgl. Kap. 6).

Bei der Orthophotoherstellung werden in der Regel digitale Geländemodelle verwendet. Objekte die höher oder tiefer als das DGM sind, werden deshalb nicht lagegenau dargestellt. Bei der Auswertung von Probekreisen im Wald kann die 
Orthorektifizierung von Luftbildern unter Verwendung eines Geländemodells wegen des deutlich höheren Kronendaches zu inventurrelevanten Verschiebungen führen (s. Abb. 14). Ein ungenaues oder nur grob aufgelöstes DGM kann ebenfalls Lagefehler verursachen (s. Abb. 15).

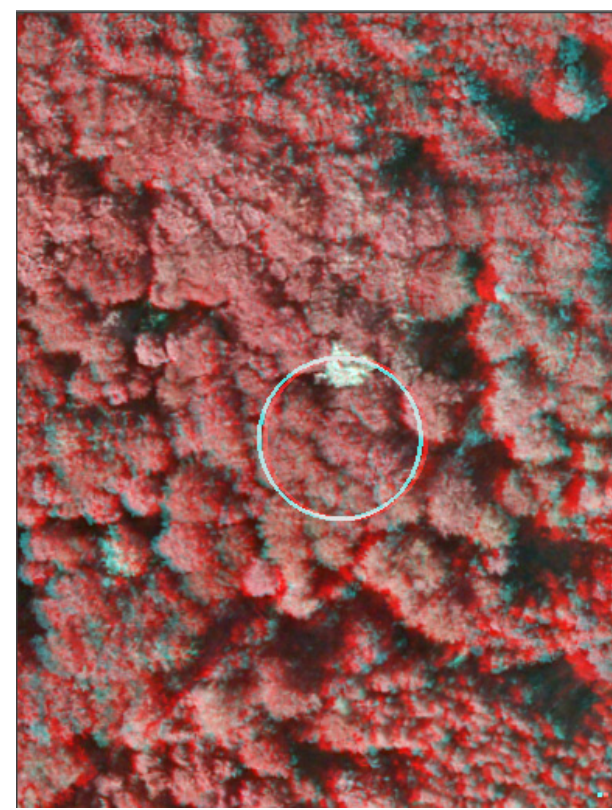

Anaglyphen-Stereo-Luftbild

Lagerichtige Kronendarstellung,

Probekreis liegt in Kronenhöhe

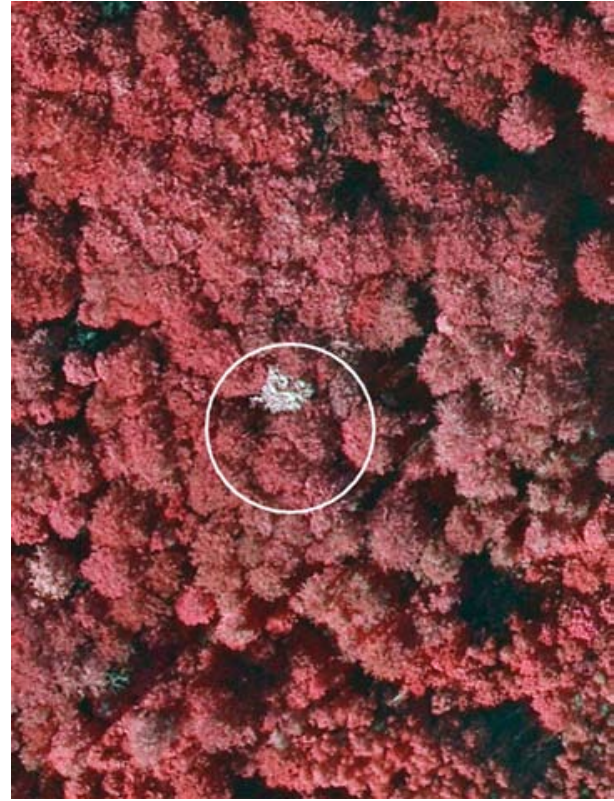

Orthophoto auf Basis DGM

Lagefalsche Kronendarstellung,

Probekreis liegt auf Geländeböhe

Abbildung 14: Kronendarstellung im Stereo-Luftbild und im DGM-basierten Orthophoto (Quelle: ATKIS®-DOP CStaatsbetrieb Geobasisinformation und Vermessung Sachsen 2012)

Die Methodik der Interpretation digitaler Orthophotos entspricht weitgehend der von analogen Orthophotos, nur erfolgt die Interpretation nun am Bildschirm. Je nach Bodenauflösung und radiometrischer Auflösung können Merkmale auf Bestandes- oder Einzelbaumebene differenziert und erfasst werden.

Die möglichen Lagefehler der kartierten Geometrien hängen von der Genauigkeit der Orthophotos ab (s. Kap. 5.3 und 6). Messfehler der Operateure können durch unabhängige Doppelmessungen quantifiziert werden (CONGALTON 1983, Gross et al. 1996). Die thematische Genauigkeit kann durch Referenzdaten oder unabhängige Doppelinterpretationen abgeschätzt werden. Der Vergleich beider Datensätze erfolgt über eine Konfusionsmatrix. Daraus kann die Gesamtgenauigkeit, die Genauigkeit aus Sicht des Nutzers und aus Sicht des Herstellers in Prozentwerten entnommen werden (siehe auch Veröffentlichung von CONGAL- 
TON et al. 1999). Durch regelmäßige Schulung und Eichung der Interpreten wird die Auswertungsqualität verbessert. Der Einsatz von Interpretationsschlüsseln ist obligatorisch (s. ARBEITSGRUPPE FORSTLICHER LUFTBILDINTERPRETEN 1998, 1999 und 2003, VEREIN DEUTSCHER INGENIEURE 1993, EUROPEAN COMMISSION 2000).

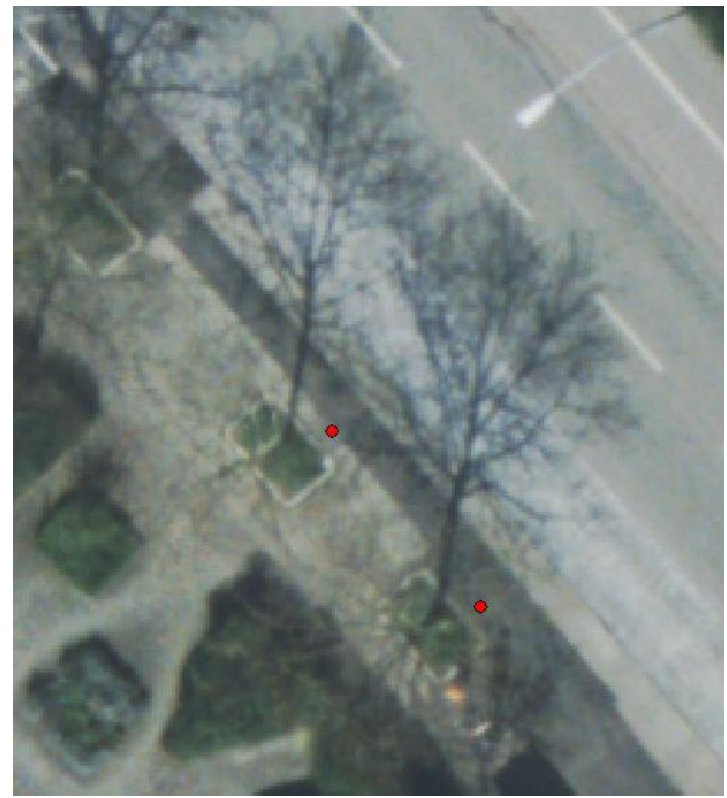

Abbildung 15: Lagefehler durch ungenaues DGM: Tatsächliche Stammfußpunkte (rot) der Bäume im Vergleich zur Lage in einem Orthophoto, das mit einem ungenauen Geländemodell berechnet wurde

\subsection{Die visuelle Auswertung digitaler Stereoluftbilder}

Die digitale Stereoluftbildauswertung (3D-Auswertung) erfordert eine spezielle photogrammetrische Hard- und Softwareausstattung (s. Kap. 9). Deren Einsatzund Auswertungsmöglichkeiten sind abhängig von:

- der Bodenauflösung

- der radiometrischen Auflösung (Farbtiefe) sowie der Anzahl und den spektralen Eigenschaften der Bildkanäle

- der Orientierungsgenauigkeit (beeinflusst die Lagegenauigkeit der Objekte sowie die Möglichkeit zur Überlagerung der Bilder mit externen Informationen)

- der Überdeckung der Einzelbilder (s. Kap. 4.3) 
Die stereoskopische 3D-Auswertung der Luftbilder bietet gegenüber der zweidimensionalen Auswertung Vorteile:

- In Luftbildern sind vor allem im Randbereich Verkippungseffekte vorhanden. In der 2D-Darstellung werden hierdurch Objekte nicht lagegenau abgebildet oder sogar verdeckt. Bei der stereoskopischen Betrachtung werden Kippeffekte ausgeglichen, so dass eine geometrisch korrekte Digitalisierung möglich ist.

- Durch den räumlichen Eindruck können Einzelbäume und Waldbestände wesentlich differenzierter und zuverlässiger interpretiert werden. Eine ausführliche Erläuterung der Stereointerpretation von Waldbeständen findet sich in dem von der Arbeitsgruppe Forstlicher Luftbildinterpreten (AFL) erarbeiteten Luftbildinterpretationsschlüssel für strukturreiche Waldbestände (ARBEITSGRUPPE FORSTLICHER LUFTBILDINTERPRETEN 2003).

- In Stereoluftbildern können Objekte nicht nur in ihrer Lage, sondern zusätzlich auch in ihrer räumlichen Ausprägung beurteilt werden.

Für die Bildauswertung ergeben sich daraus folgende Konsequenzen:

- Es ist eine differenziertere Interpretation und Abgrenzung von Waldbeständen nach deren Baumartenzusammensetzung und Strukturmerkmalen möglich. Bei entsprechender geometrischer Auflösung können deutlich ausgeprägte Baumkronen (Kraft'sche Baumklassen 1 bis 3) erfasst und abgegrenzt werden. Bei Einzelkronen können Aussagen über die Baumart, den Zustand und die Struktur getroffen werden.

- Die Lage der erfassten Objekte wird von einem geübten Auswerter in Abhängigkeit von Bildmaterial und Bildorientierung genau gemessen. Das Problem der Verkippung der Baumkronen auf Orthophotos, die mit einem Geländemodell und nicht mit einem Oberflächenmodell berechnet wurden, wird umgangen.

- Je nach Bodensichtbarkeit können Bodenpunkte gemessen und daraus Geländemodelle gerechnet werden.

- Falls ein Geländemodell vorliegt oder Bodensicht vorhanden ist, kann die Baumhöhe mit denselben Einschränkungen wie bei der analogen Stereobildauswertung erfasst werden. Nach HILDEBRANDT (1996) sind die wichtigsten Faktoren, welche die Höhenmessung von Baum und Bestand beeinflussen:

○ die Struktur und Form der Einzelkronen

○ der Aufnahmezeitpunkt (keine Messungen im entlaubten Zustand)

○ die Fußpunktmessung (Bodensicht, Bodenvegetation, Steilheit des Geländes) 
- In Abhängigkeit von Geländebeschaffenheit, Bildqualität und Programmfunktionen können Oberflächen- und/oder Geländemodelle automatisiert bzw. teilautomatisiert erstellt werden. Eine Plausibilitätskontrolle der berechneten Höhenmodelle ist in jedem Fall notwendig. Hierzu können in den meisten Programmen die Höhenmodelle dreidimensional visualisiert und editiert werden.

Die stereoskopische Auswertung ist im Vergleich zur Orthophotoauswertung leistungsfähiger, aber zeitaufwendiger und kostenintensiver und sie stellt höhere Anforderungen an Erfahrung und Sachkenntnis der Interpreten.

Die möglichen Lagefehler der gemessenen Geometrien hängen wie bei der Auswertung von Orthophotos von der Genauigkeit der Aerotriangulation ab (s. Kap. 5.3). Zusätzliche Messfehler der Operateure in Lage und Höhe können durch unabhängige Doppelmessungen quantifiziert werden (CONGALTON 1983, GROSs et al. 1996). Bei der thematischen Genauigkeit und Qualitätssicherung gilt das gleiche wie bei der visuellen Auswertung digitaler Orthophotos (s. Kap. 7.1).

\subsection{Die teilautomatisierte Auswertung digitaler Luftbilder}

Vollautomatisch ablaufende Auswerteprozesse für Waldvegetation sind aufgrund der sehr unterschiedlichen Eigenschaften von Luftbildern (z. B. Bodenauflösung, Beleuchtung) nicht möglich. Allerdings können im Rahmen derartiger Prozesse einzelne Arbeitsschritte (z. B. Berechnung von Oberflächenmodellen, Waldlückendetektion) automatisiert durchgeführt werden.

Charakteristika einer automatisierten Auswertung sind:

- Übertragbarkeit auf andere Datensätze

- keine manuellen Eingriffe während des Auswertungsprozesses

- keine Nachbereitung der Ergebnisse

Bei einer teilautomatisierten Auswertung sind korrektive Maßnahmen Teil des Gesamtprozesses.

Vorteile (teil-)automatisierter Prozesse sind:

- große Gebiete können rationell und schnell ausgewertet werden

- höherer Standardisierungsgrad

- höhere Homogenität und Objektivität der Ergebnisse

- Wiederholbarkeit der Arbeitsabläufe

Nachteile (teil-)automatisierter Prozesse:

- komplexes Wissen und spezielle Software notwendig 
- Gefahr verringerter Transparenz aufgrund komplexer Zusammenhänge und Algorithmen

- Abstriche beim Detaillierungsgrad der Zielsetzung

Die (teil-)automatisierte Auswertung von digitalen Luftbildern unterscheidet folgende aufeinander aufbauende Arbeitsschritte:

Bei der Datenvorbereitung liegen die Schwerpunkte auf der Verringerung der atmosphärischen Einflüsse, dem Ausgleich der topographischen und bildortsabhängigen Beleuchtungsverhältnisse (s. Abb. 16) sowie der Reduktion der redundanten Bildinformationen (z. B. PCA, NDVI). Zusätzlich kann es notwendig sein, hohe Bodenauflösungen durch ein Resamplingverfahren zu verringern.
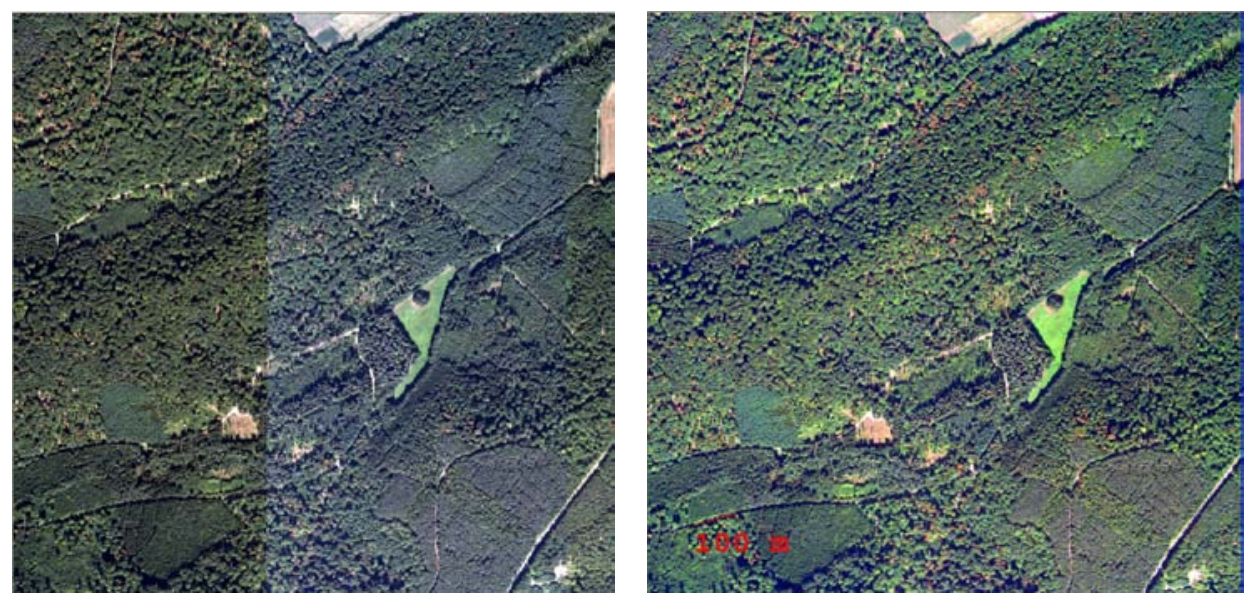

Abbildung 16: Bildhomogenisierung durch Beleuchtungskorrektur - links: Originalbilder, rechts: homogenisierte Bilder (Quelle: Bayerisches Landesamt für Vermessung und Geoinformation)

Bei der Datenauswertung unterscheidet man pixelbasierte und objektorientierte Verfahren. Zusätzlich können wissensbasierte Verfahren integriert werden (s. auch LU et al. 2007).

Pixelbasierte Verfahren weisen jedes einzelne Pixel aufgrund seiner spektralen Eigenschaften einer Klasse zu. Nachbarschaften werden nicht berücksichtigt. Objektorientierte Klassifizierungsverfahren beruhen darauf, dass über vom Nutzer aufgestellte Regelwerke aus Pixeln Bildsegmente berechnet und anhand ihrer Textur, ihrer spektralen und geometrischen Eigenschaften sowie gegenseitigen Beziehungen definierten Klassen zugewiesen werden. Bei digitalen Luftbildern werden aufgrund der hohen Variabilität der spektralen Eigenschaften in einem Objekt in der Regel objektorientierte Verfahren angewandt (s. Abb. 17). Eine detaillierte Beschreibung der Auswertungsmethoden kann bei ALBERTZ (2007) nachgelesen werden. 


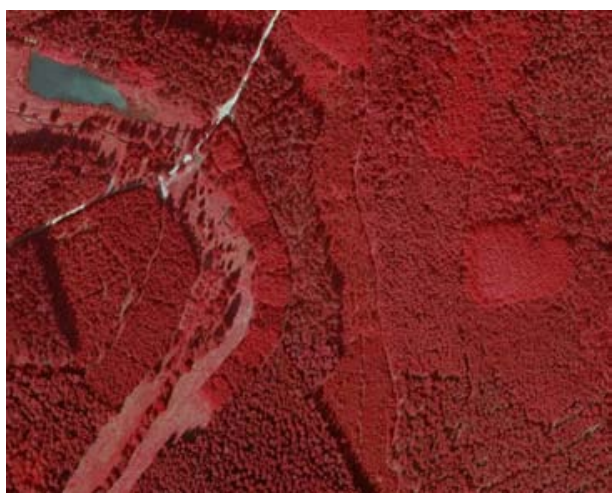

Orthophoto

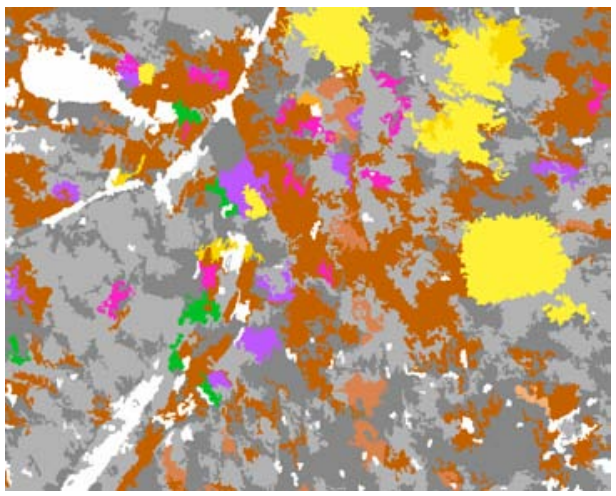

Klassifizierungsergebnis

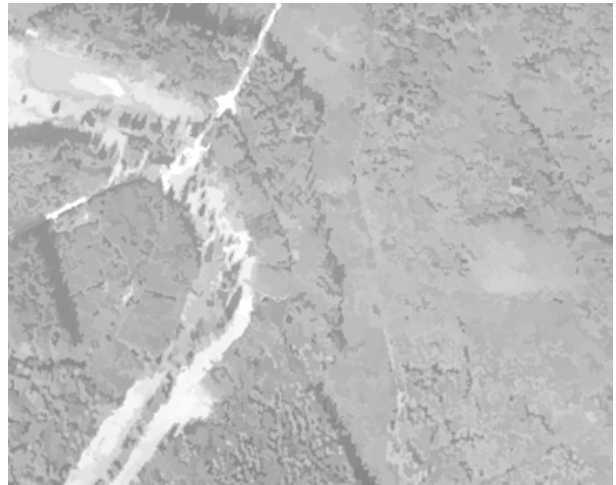

Segmentierungsergebnis

Baumartengruppen und Wuchsklassen

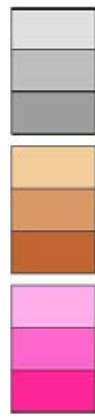

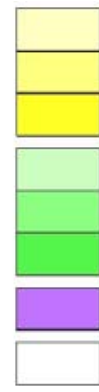

Eiche - Jungwuchs

Eiche $\cdot$ Stangenholz

Eiche - Baumholz

Buche - Jungwuchs

Buche - Stangenhot?

Buche - Baumholz

Sonstige Weichlaub:

unikiass fiziert

Abbildung 17: Objektbasierte Klassifikation eines Orthophotos (GRIEßMANN 2007; Datenquelle DOP: Sachsenweite Befliegung 2005, ATKIS ${ }^{\circledR}-D O P$, (C) Staatsbetrieb Geobasisinformation und Vermessung Sachsen 2012)

Nach abgeschlossener Klassifizierung sollte stets eine Verifizierung des Ergebnisses erfolgen. Dazu werden unabhängige Referenzdaten herangezogen. Diese können sowohl im Gelände erhoben als auch aus aktuellen Geofachdaten gewonnen werden. Der Vergleich beider Datensätze erfolgt über eine Konfusionsmatrix. Daraus kann die Gesamtgenauigkeit, die Genauigkeit aus Sicht des Nutzers und aus Sicht des Herstellers in Prozentwerten entnommen werden (s. CONGALTON et al. 1999). 


\subsection{Aktuelle praktische Beispiele}

1) Im Schweizerischen Landesforstinventar werden mittels Stereointerpretation von ADS40-SH52- und ADS80-SH82-Bildstreifen (25 und $50 \mathrm{~cm}$ GSD) in einem Stichprobennetz an 22.000 Probeflächen Informationen zur Bodenbedeckung interpretiert. Daraus werden unter anderem Bestockungsgrade, Waldflächen nach unterschiedlichen Definitionen und Angaben zur Gehölzbiomasse abgeleitet. Verwendet wird die Software StereoAnalyst in einer ArcGIS-Umgebung.

Literaturhinweis:

Hastedt, H.; Meile, R.; Ginzler, C. (2009): ArcGIS Applikation zur 3D-Datenerfassung im Landesforstinventar der Schweiz. In: Strobl, J.; Blaschke, T.; Griesebner, G. (Hrsg.): Angewandte Geoinformatik 2009. Beiträge zum 21. AGIT-Symposium Salzburg. Heidelberg, Herbert Wichmann, 8-13.

2) Im Projekt „Wirkungskontrolle Moorbiotope Schweiž“ werden in Stereo-Luftbildern (ADS40, Ultracam, digitalisierte RC30) homogene Einheitsflächen kartiert. Aus diesen Einheitsflächen wird anhand der Bildinformation (Farben) und den gerechneten Oberflächenmodellen eine Stichprobe gezogen. In diesen Flächen werden terrestrisch Artenlisten aufgenommen, die anschließend anhand der Bildinformationen auf die gesamte Fläche modelliert werden. Das Monitoring wird alle 6 Jahre wiederholt. Die photogrammetrischen Datensätze werden mit SocetSet erstellt.

Literaturhinweis:

Ecker, K.; Küchler, M.; Feldmeyer-Christe, E.; Graf, U.; Waser, L. T. (2008): Predictive mapping of floristic site conditions across mire habitats: Evaluating data requirements. Community Ecol. 9, 2, 133-146.

3) Bei einem Waldbrand oberhalb Leuk im Kanton Wallis brannten 2003 über 300 ha Wald zwischen 750 und $1400 \mathrm{~m}$ ü. NN. Durch Stereointerpretation von digitalisierten CIR-RC30-Bildern ( 1 : 5.000) wird die unterschiedliche Entwicklung der vier Hauptbaumarten (Eiche, Fichte, Kiefer, Lärche) nach dem Brand jährlich wiederholt erfasst. Die Interpretation erfolgt mit SocetSet für ArcGIS.

Literaturhinweis:

Wohlgemuth, T.; Brigger, A.; Gerold, P.; Laranjeiro, L.; Moretti, M.; Moser, B.; Rebetez, M.; Schmatz, D.; Schneiter, G.; Sciacca, S.; Sierro, A.; Weibel, P.; Zumbrunnen, T.; Conedera, M. (2010): Leben mit Waldbrand. Merkbl. Prax. 46, WSL Birmensdorf, $16 \mathrm{~S}$. 
4) Für den Kanton Zürich werden $W$ aldbestandeskarten mittels digitaler Photogrammetrie erstellt. Zu diesem Zweck werden die Waldregionen mit CIR-Luftbildern beflogen (digitalisierte RC 30-Bilder, Ultracam). Die Waldbestandesdaten dienen als wesentliche Grundlage für die Waldentwicklungs- und Betriebsplanung. Hierzu werden die luftbildsichtbaren Bestandesmerkmale wie Entwicklungsstufe, Hauptbaumarten, Struktur, Schlussgrad und Mischungsgrad durch einen erfahrenen Interpreten direkt an einer digitalen Photogrammetriestation (SocetSet) erfasst. Die mittlere Bestandeshöhe wird durch Baumhöhenmessungen ermittelt. Als Nebenprodukte werden Oberflächenmodelle und Orthophotos gerechnet. Dieses bewährte Standardverfahren wird leicht modifiziert auch in weiteren Kantonen angewendet.

Ansprechpartner:

Markus Wortmann, Scherrer Ingenieurbüro AG, Hauptstrasse 14, CH-9650 Nesslau, Telefon: 0041 (71) / 99422 44, Email: scherrerag@bluewin.ch

5) Zur Erfassung und Bewertung der räumlichen Strukturdiversität in Waldbeständen wurden luftbildsichtbare Strukturparameter erhoben, die als Diversitätsindikatoren geeignet sind. Die visuelle Interpretation und die Digitalisierung der Strukturparameter erfolgten mit StereoAnalyst für ArcGIS auf der Basis einer Zeitreihe von digitalisierten Color-Infrarot-Luftbildern. Parallel wurden zu Validierungszwecken die analogen Original-Luftbilder an einem analogen Stereoskop begutachtet.

Literaturhinweis:

Engels, F. (2009): Erfassung und Bewertung der räumlichen Strukturdiversität in Level II Beständen mit Hilfe von Color-Infrarot-Luftbildern. PDF-Download in der Internetpräsentation der FAWF unter www.fawf.wald-rlp.de unter „Unser Team“, „Engels Friedrich“, Projekt „Erfassung und Bewertung ...“, 76 S.

6) Zur Erfassung und Bewertung von Natura 2000 Wald-Lebensraumtypen im Bayerischen Hochgebirge wird auf der Grundlage einer vorhandenen Potentialkartierung durch die visuelle Interpretation von stereoskopischen, digitalen Farbinfrarot-Luftbildern in einem zweistufigen Verfahren zunächst die Lebensraumtypeneigenschaft von Wäldern in Hochgebirgs-FFH-Gebieten überprüft. In einem weiteren Schritt wird deren Erhaltungszustand in einer dreistufigen Skala bewertet. In beiden Anwendungsfällen kann der Aufwand für kostenintensive Geländeaufnahmen bei ausreichender Aussageschärfe entscheidend reduziert werden.

Ansprechpartner:

Rudolf Seitz und Armin Troycke, Bayerische Landesanstalt für Wald und Forstwirtschaft (LWF), Abteilung Informationstechnologie, Hans-Carl-von-CarlowitzPlatz 1, D-85354 Freising, Telefon: 0049 (8161)/71-4963 
7) Im Rahmen eines Projektvorhabens wird die semi-automatische Extraktion forstlicher Parameter (Baumart, Mischung, Bestandeshöhen, Entwicklungsphasen, Beschirmungsgrad und Lückigkeit) aus digitalen Luftbildern (großformatige Matrixkamera) untersucht. Hierzu werden ausschließlich amtliche Luftbilddaten des Bayerischen Landesamtes für Vermessung und Geoinformation (LVG) verwendet. Erste Zwischenergebnisse weisen auf das große Potential der amtlichen Luftbilddaten hin.

Ansprechpartner:

Rudolf Seitz und Dr. Christoph Straub, Bayerische Landesanstalt für Wald und Forstwirtschaft (LWF), Abteilung Informationstechnologie, Hans-Carl-von-Carlowitz-Platz 1, D-85354 Freising, Telefon: 0049 (8161) / 71-4963

8) Das Heidemonitoring für die Bewertung mehrerer FFH-Lebensraumtypen in Brandenburg wird routinemäßig mit Hilfe weitgehend automatisierter Auswertung von Fernerkundungsdaten durchgeführt. Wichtigstes Bewertungskriterium ist die Zusammensetzung der Vegetationsdecke, darunter das Aufkommen von Waldbaumarten als negatives Merkmal. Höchstauflösende Satellitendaten (Quickbird) als bevorzugte Datengrundlage sind nicht immer und überall verfügbar. Versuche, an deren Stelle digitale Luftbilder automatisch zu klassifizieren, waren nur teilweise erfolgreich, weil bildortabhängige Beleuchtungsunterschiede als Störeinfluss nicht vollständig eliminiert werden konnten.

Ansprechpartner:

Gregor Weyer, LUP-Umwelt, Große Weinmeisterstr.3a, D-14469 Potsdam, Email: gregor.weyer@lup-umwelt.de

Literaturhinweise:

Frick, A. (2005): Evaluation of Natura 2000 habitat types through very high resolution satellite imagery. In: Göttinger Geographische Abhandlungen, Heft 113, GGRS 2004, 1st Göttingen GIS \& Remote Sensing Days.

Frick, A. u. Kenneweg, H. (2010): Assessment and Monitoring of Natura-2000Habitat Types Using Semi-Automatic Processing of EO-Satellite Data. In: NEREUS Earth Observation / GMES working Group (Editor): 25 Uses of GMES in the NEREUS Regions, S. 1-2. 
9) Im Jahr 2007 hat eine Erfassung von Sturmschäden in Nordrhein-Westfalen stattgefunden. Mit Hilfe von digitalen Orthophotos und Stereo-Luftbildern wurden auf einem Drittel der Landesfläche Nordrhein-Westfalens die durch den Orkan Kyrill geschädigten Waldflächen erfasst. In dem Artikel werden die verwendeten Methoden beschrieben und die ersten Ergebnisse dargestellt.

Ansprechpartner:

Frank Franken, Hessen-Forst FENA, Europastraße 10-12, D-35394 Gießen, Telefon: 0049 (641)/4991-257, Email: frank.franken@forst.hessen.de

Literaturhinweis:

Franken, F.; Franz, S. u. Müterthies, A. (2007): Erfassung der durch den Orkan Kyrill geschädigten Waldgebiete in Nordrhein-Westfalen anhand von digitalen Luftbildern und Orthophotos.-PFG, Heft 5, S. 349-354.

10) Zur Erstellung von Gerichtsgutachten im Rahmen von Landrechtsfällen in Sarawak (Malaysia) werden historische Luftbilder von vor 1953 digitalisiert und ausgewertet. Die Schwarzweiß-Luftbilder sind in England archiviert und zugänglich. Nach dem Scannen werden diese absolut orientiert und danach in einer digitalen Arbeitsumgebung (ArcGIS mit StereoAnalyst) interpretiert. Ziel ist dabei der Nachweis / Nicht-Nachweis menschlicher Aktivitäten in den jeweiligen Fallgebieten vor 1953. Kriterien sind Eingriffe in den Primärwald, Landwirtschaft oder auch Langhäuser und Fußpfade.

Ansprechpartner:

Dr. C. P. Gross, Albert-Ludwigs Universität Freiburg, Abteilung Fernerkundung und Landschaftsinformationssysteme, D-79085 Freiburg. Tel.: 0049 (761)/ 2033696,Email: gross@felis.uni-freiburg.de

11) Für die Beobachtung von Naturwaldzellen in Sachsen wurden digitale Luftbilder eingesetzt. Zusammen mit Daten des Forstlichen Informationssystems Sachsens sowie terrestrischen Erhebungen und Interpretationsergebnissen analoger Luftbilder wurden Bilder von DMC und UltraCam-D stereoskopisch sowie als Orthophotos ausgewertet. Bei der Erfassung von Waldzustandsparametern (Deckungsgrad, Schichtung, Wuchsklassen, Mischung,...) in Stichprobenkreisen bewährte sich der Luftbild-Interpretationsschlüssel für strukturreiche Waldbestände (ARBEITSGRUPPE FORSTLICHER LUFTBILDINTERPRETEN 2003). Die Auswertung mittels StereoAnalyst (ArcGIS) war für die Beschreibung der Parameter zu unterschiedlichen Aufnahmezeitpunkten in exakt positionierten Probekreisen und für die Feststellung von Veränderungen sehr gut geeignet.

Literaturhinweis:

Hoffmann, K. (2010): Erfassung von Waldstrukturen und Waldzustandsparametern aus digitalen Luftbilddaten der sachsenweiten Befliegung 2005. Forstliche Forschungsberichte München, Heft 209, S. 18-29. 
12) Ein Verfahren zur automatisierten Baumartenklassifizierung unter Verwendung von digitalen Luftbilddaten (DMC, UltraCam-D) und einem objektorientierten Verfahrensansatz (Definiens Professional) wurde im Rahmen einer Diplomarbeit für ausgewählte sächsische Testgebiete entwickelt. Baumartengruppen und die Wuchsklassen von Hauptbaumarten waren gut erkennbar. Schwierigkeiten gab es bei der Erfassung der Nebenbaumarten.

Literaturhinweis:

Grießmann, P. (2007): Erfassung von Waldstruktur- und Waldzustandsparametern aus Digitalen Luftbilddaten der sachsenweiten Befliegung 2005. Diplomarbeit, TU Dresden, Institut für Kartographie.

13) Die Erfassung von Waldflächen in Sachsen, welche bisher nicht in der Forstgrundkarte $1: 5.000$ dargestellt waren, erfolgte im Auftrag des Staatsbetriebes Sachsenforst in den Jahren 2005/06 auf der Basis von digitalen CIR-Orthophotos (DMC, UltraCam-D; GSD 0,2 m auf 0,8 $\mathrm{m}$ resampelt). Die Waldflächen wurden nach Vorgabe des Sächsischen Waldgesetzes auf Luftbildkarten abgegrenzt und unter Verwendung von ArcInfo am Bildschirm digitalisiert. Für alle sächsischen Forstbezirke wurden so aktuelle CIR-Luftbildkarten $1: 5.000$ hergestellt.

Literaturhinweis:

Hoffmann, K. (2005): Erfassung von Waldflächen mit Hilfe von Luftbildern. Forst Journal 02/2005, S. 2-3, Herausgeber: Sächsisches Staatsministerium für Umwelt und Landwirtschaft.

14) In Orthophotos wurden die Auswirkungen unterschiedlicher Walddefinitionen untersucht. Dabei wurde die nationale Definition Österreichs der international üblichen Definition der Food Agriculture Organization (FAO) gegenübergestellt. Für die Erhebungsteams der nationalen Waldinventur 2007/2009 sollte diese Klassifikation als Vorstudie die im alpinen Gelände oft schwierige Anwendung der Flächenund Überschirmungskriterien nach FAO erleichtern. In festen Probekreisen von 0,5 ha wurde die Wald-/Nichtwald-Entscheidung an 22.000 Stichprobenpunkten durch visuelle Interpretation in einer GIS Umgebung durchgeführt. Die Differenz zwischen den Ergebnissen beider Erhebungen ist gering und beträgt rund 1\%.

Literaturhinweise:

Tomter, S. M.; Bonhomme, C.; Gabler, K.; Gagliano, C.; Gasparini, P.; Gschwantner, T.; Kroiher, F.; Polley, H.; Robert, N.; Rondeux, J.; Schadauer, K.; Schmitz, F.; Vidal, C. (2011): Towards Bridge Building Procedures for Forest Area Estimates: Examples from European National Forest Inventories (Forest Science, in Review).

Hauk, E.; Schadauer, K. (2005): Terms and definitions for the national reporting tables for FRA 2005

www.fao.org/forestry/fra/2005/terms/en/

Beiträge aus der NW-FVA, Band 7, 2012 
15) An der Forstlichen Versuchs- und Forschungsanstalt Baden-Württemberg wurde ein teilautomatisiertes Stichprobenverfahren zur Abschätzung der Koblenstoffspeicherung in Stadtbäumen entwickelt. Es werden Stereoluftbilder, Orthophotos und ALS-Daten verwendet. Hierbei wird die von Baumkronen überschirmte Fläche im Stadtgebiet automatisch abgeleitet. Zufällig ausgewählte Stichprobenbäume werden stereoskopisch vermessen. Diese Parameter dienen zur Modellierung des Kohlenstoffvorrats im Stadtgebiet.

Ansprechpartner:

Dr. Petra Adler, Forstliche Versuchs- und Forschungsanstalt Baden-Württemberg (FVA), Abteilung Biometrie und Informatik, Wonnhaldestraße 4, D-79100 Freiburg, Telefon 0049 (761)/4018-207

16) In Potsdam wurde ein Stadtbaummonitoring entwickelt, in dessen Rahmen Eigenschaften von Stadtbäumen (Baumhöhe, Kronenumriss, Kronenvolumen, Vitalitätszustand usw.) sowie deren Veränderungen aus Stereo-Luftbildern ermittelt und im städtischen Baumkataster dokumentiert werden. Die Baumartenerkennung kann aus unterschiedlichen Gründen schwierig sein, die Feststellung der Verkehrssicherheit ist i. d. R. nicht möglich. Zusätzlich verwendet die Stadt Potsdam diese Daten im Zusammenhang mit Klimaschutzmaßnahmen und hat sie um die stärker generalisierte und weitgehend automatisierte Ermittlung des Grünvolumens und dessen Veränderung (z. B. für die Befundeinheit ,,statistischer Block“") erweitert.

Literaturhinweis:

Arge Integriertes Klimaschutzkonzept für die Landeshauptstadt Potsdam (2010): Gutachten zum Integrierten Klimaschutzkonzept 2010, Kapitel 10 - Handlungsfeld Landschafts- und Umweltplanung, S. 141-215.

17) Für die Identifizierung von Vitalitätskennwerten wurde im August 2004 ein Traubeneichenbestand mit der UltraCam D (10 cm GSD) beflogen. Die Überlappung in Quer- und Längsrichtung wurde so hoch gewählt, dass jeder Referenzbaum in mehr als 8 Luftbildern abgebildet war. Damit konnte der gleiche Baum bei verschiedenen Beleuchtungssituationen untersucht werden. In den Luftbildern wurden Kronenpolygone stereoskopisch digitalisiert und in 4 Teile unterteilt. Zeitgleich wurde an den Baumkronen eine terrestrische Schadansprache durchgeführt. Zur Beurteilung des Einflusses der Lage der Testbäume auf den Bildern auf die Schadstufen wurden die Grauwertinformationen der Gesamtkronen und deren Teile statistisch ausgewertet. Zudem wurden bekannte Indizes (NDVI etc.) auf ihre Aussagekraft für die Schadansprache unter wechselnden Aufnahmebedingungen untersucht.

Ansprechpartner:

Kai Jütte, Landesforst Mecklenburg-Vorpommern - Anstalt des öffentlichen Rechts; Betriebsteil: Forstplanung, Versuchswesen, Forstliche Informationssyste- 
me; Sachgebiet: Fernerkundung; Zeppelinstraße 3, D-19061 Schwerin, Telefon: 0049 (385)/6700-227

Literaturhinweis:

Anders, G. (2006): Identifikation von Vitalitätskennwerten mittels digitaler Luftbilddaten. Master Thesis Abschlussarbeit Universität Salzburg.

18) Im Buchennaturwaldreservat Heilige Hallen (Mecklenburg-Vorpommern) wurde eine Luftbildzeitreibe aller verfügbaren Luftbilder von 1938 bis 2007 erstellt und ausgewertet. Dabei wurden die Kronengröße, die Baumanzahl, die Veränderung der Verjüngungsfläche, die Lage und Anzahl des Totholzes digital stereoskopisch kartiert. Durch den maximalen Abstand von 10 Jahren zwischen aufeinander folgenden Luftbildjahrgängen konnte der Wechsel von der Optimal- zur Zerfallsphase detailliert dokumentiert werden. Eine besondere Herausforderung war die sehr unterschiedliche Qualität der Ausgangsdaten, die von analogen SchwarzweißLuftbildern mit sehr geringer Auflösung bis zu digitalen Luftbildern mit $10 \mathrm{~cm}$ Bodenauflösung reichte.

Ansprechpartner:

Kai Jütte, Landesforst Mecklenburg-Vorpommern - Anstalt des öffentlichen Rechts; Betriebsteil: Forstplanung, Versuchswesen, Forstliche Informationssysteme; Sachgebiet: Fernerkundung; Zeppelinstraße 3, D-19061 Schwerin, Telefon: 0049 (385)/6700-227

19) In mehreren Projekten wurde die Nutæung von Digitalen Luftbildern von verschiedenen Flugplattformen (Drohne, Kleinflugzeug) und Sensoren (Kleinformatkamera, Mittelformatkamera, Großformatkamera, Laserscanner) für verschiedene Anwendungen in der Forstwirtschaft getestet. Dabei wurde die Genauigkeit von Baumhöhenmessungen im Stereomodell, die Erkennbarkeit von Baumarten in strukturreichen Naturwäldern in Laserdaten und in Luftbildern zum Zeitpunkt der Laubverfärbung untersucht. Weitere Themen waren die zeitnahe und schnelle Erhebung von Beweismitteln und deren Genauigkeit nach forstlichen Eingriffen (Bestockungsgradabsenkungen) sowie die Kartierung von Nadelverlusten durch Kiefernbuschhornblattwespen in UltraCamXp-Daten.

Ansprechpartner:

Kai Jütte, Landesforst Mecklenburg-Vorpommern - Anstalt des öffentlichen Rechts; Betriebsteil: Forstplanung, Versuchswesen, Forstliche Informationssysteme; Sachgebiet: Fernerkundung; Zeppelinstraße 3, D-19061 Schwerin, Telefon: 0049 (385)/6700-227

Literaturhinweis:

Jütte, K.; Rüffer, O. (2010): Nutzung moderner Fernerkundungsverfahren für forstliche Fragestellungen - Wissenstransfer in die Praxis. Eberswalder Forstliche Schriftenreihe Band 44, S. 28-37. 
20) An der Nordwestdeutschen Forstlichen Versuchsanstalt werden digitale Luftbilder intensiv im Bereich Waldschutz, eingesetzt. Schwerpunkte sind die Erfassung von Borkenkäferschäden, Nadelfraßschäden durch Kieferninsekten, Sturmschäden und Nadel-/Blattverluste oder Absterbeerscheinungen in Waldbeständen. Hinzu kommt die Ausschlussflächenkartierung im Falle der Ausbringung von Pflanzenschutzmitteln. Es werden Luftbilder aller marktgängigen großformatigen Matrixkameras verwendet. Interpretiert werden Orthophotos oder Stereomodelle.

Ansprechpartner:

Jörg Ackermann, Nordwestdeutsche Forstliche Versuchsanstalt, Grätzelstraße 2, D37079 Göttingen, Telefon: 0049 (551)/69401-127, Email: joerg.ackermann@nwfva.de

21) Von der Thüringer Landesanstalt für Wald, Jagd und Fischerei (seit 01.01.2012: ThüringenForst - Anstalt öffentlichen Rechts, Service und Kompetenzzentrum) wurden Bildmosaike zur visuellen Sturmscbadenskartierung (Einzelbrüche und Einzelwürfe) im Forstamt Bad Salzungen genutzt. Die Bilddaten werden mit einem selbstständig fliegenden Kleinstflugzeug (Drohne) und einer Kleinformatkamera aufgenommen und anschließend ohne Triangulation zu einem Bildmosaik zusammengesetzt, das schließlich georeferenziert wird. Alle Arbeitsschritte sind standardisiert und weitgehend automatisiert, so dass die Bildmosaike wenige Arbeitstage nach der Befliegung für die Revierleiter zur Verfügung stehen. Die Technologie wurde im Rahmen des ANDROMEDA®-Projektes entwickelt.

Literaturhinweis:

Sagischewski, H.; Chmara, S.; Uth, J. u. Sossna, I. (2010): Erfassung von Sturmschäden mithilfe von ANDROMEDA®-Daten. AFZ-Der Wald, Heft 21, S. 6-8. 



\section{Datenmanagement}

Digitale Luftbilder stellen durch ihre großen Datenmengen besondere Ansprüche an Speicherung, Dokumentation (Metadaten), Handhabung sowie Archivierung und erfordern sehr leistungsfähige Hard- und Softwaresysteme.

\subsection{Konzepte der Datenhaltung}

Es gibt verschiedene Möglichkeiten der digitalen Datenhaltung (s. Abb. 18).

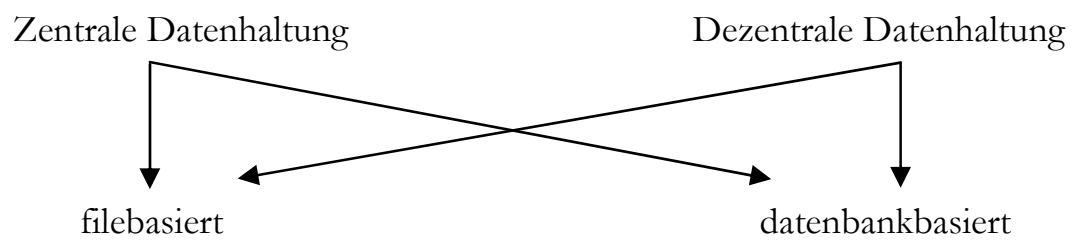

Abbildung 18: Arten der digitalen Datenhaltung

Jede der oben dargestellten Kombinationen ist für die Speicherung großer digitaler Datenmengen denkbar, aber im konkreten Fall mehr oder weniger gut geeignet. Grundsätzlich muss die Datenstruktur sorgfältig vorausgeplant werden, da spätere Änderungen erheblichen Aufwand für alle Anwender nach sich ziehen können.

$\mathrm{Zu}$ beachten sind dabei auch Pfad- und Dateinamen, da nicht jede Software Sonderzeichen, Leerzeichen oder lange Dateinamen verarbeiten kann.

\subsubsection{Zentrale, filebasierte Datenhaltung}

Die Daten werden auf einem Server als Einzeldateien gespeichert. Nutzer bekommen über ein Netzwerk darauf Zugriff. Dies ist ein einfaches, noch häufig in Verwendung befindliches Verfahren, das immer mehr durch datenbankbasierte Systeme abgelöst wird. 
Tabelle 12: Vor- und Nachteile einer zentralen, filebasierten Datenhaltung

\begin{tabular}{l|l}
\hline Vorteile & Nachteile \\
\hline$+\begin{array}{l}\text { geringer Aufwand erforderlich, um } \\
\text { ein funktionsfähiges System zu er- } \\
\text { stellen }\end{array}$ & $\begin{array}{l}\text { - hohe Anforderungen an Datenstruk- } \\
\text { turierung und -dokumentation } \\
\text { (Metadatensystem) }\end{array}$ \\
$\begin{array}{l}\text { einfach zu verwalten und zu } \\
\text { pflegen (keine Datenbankkennt- }\end{array}$ & $\begin{array}{l}\text { - eine Auswahl und Abfrage anhand } \\
\text { räumlicher oder thematischer Krite- } \\
\text { rien ist nur schwer möglich }\end{array}$ \\
$+\begin{array}{l}\text { preisgünstig (Kosten hängen fast } \\
\text { ausschließlich von der Hardware } \\
\text { ab) }\end{array}$ & $\begin{array}{l}\text { die Verwaltung der Zugriffsrechte ist } \\
+ \text { einfacher Datenzugriff } \\
+\begin{array}{l}\text { viele Nutzer können gleich } \\
\text { Daten lesen }\end{array}\end{array}$ \\
\hline
\end{tabular}

\subsubsection{Zentrale, datenbankbasierte Datenhaltung}

Die Daten werden auf einem Server in einer Datenbank gespeichert. Die Nutzer haben Zugriff über ein Netzwerk mit individuellen Zugriffsrechten. Diese Art der Datenhaltung bietet sich für große und komplexe Datenbestände an.

Tabelle 13: Vor-und Nachteile einer zentralen, datenbankbasierten Datenhaltung

\begin{tabular}{|c|c|}
\hline Vorteile & Nachteile \\
\hline $\begin{aligned} & \text { einfacher und schneller Zugriff auf } \\
& \text { alle Daten durch thematische oder } \\
& \text { räumliche Suchfunktion } \\
+ & \text { komplexe Auswertungen möglich } \\
+ & \text { integriertes und leistungsfähiges } \\
& \text { Management der Zugriffsrechte } \\
+ & \text { Vermeidung von Datenredun- } \\
& \text { danzen } \\
+ & \text { einfachere und bessere Möglich- } \\
& \text { keiten der Datensicherung }\end{aligned}$ & $\begin{array}{l}\text { - Spezialwissen über Datenbank- } \\
\text { administration erforderlich } \\
\text { - höherer Aufwand für Installation, } \\
\text { Verwaltung und Pflege } \\
\text { - höhere Kosten durch Datenbank- } \\
\text { software } \\
\text { - Zugriff nur über entsprechende Pro- } \\
\text { gramme }\end{array}$ \\
\hline
\end{tabular}

\subsubsection{Dezentrale, filebasierte Datenhaltung}

Die Daten werden auf lokalen Rechnern in Form von Einzeldateien gespeichert. Die Nutzer haben direkten Zugriff auf die Daten mit ihnen zugeordneten Zugriffsrechten. Dieses Verfahren wird bei Datenleitungen mit geringem Datentransfervolumen eingesetzt. 
Tabelle 14: Vor- und Nachteile einer dezentralen, filebasierten Datenhaltung

\begin{tabular}{|c|c|}
\hline Vorteile & Nachteile \\
\hline $\begin{aligned} &+ \text { speicherintensive Daten belasten } \\
& \text { nicht das Netz } \\
&+ \text { sehr einfacher und schneller } \\
& \text { Zugriff auf die Daten des eigenen } \\
& \text { Rechners } \\
&+ \text { geringer Aufwand erforderlich, um } \\
& \text { ein funktionsfähiges System zu } \\
& \text { erstellen } \\
&+ \text { einfach zu verwalten und zu } \\
& \text { pflegen (keine Datenbankkennt- } \\
& \text { nisse notwendig) } \\
&+ \text { peisgünstig (Kosten hängen fast } \\
& \text { ausschließlich von der Hardware } \\
& \text { ab) }\end{aligned}$ & $\begin{array}{l}\text { - hohe Anforderungen an Datenstruk- } \\
\text { turierung und -dokumentation } \\
\text { (Metadatensystem) } \\
\text { - Datenredundanz wahrscheinlich } \\
\text { - eine Auswahl und Abfrage anhand } \\
\text { räumlicher oder thematischer Krite- } \\
\text { rien ist nur schwer möglich }\end{array}$ \\
\hline
\end{tabular}

\subsubsection{Dezentrale, datenbankbasierte Datenhaltung}

Die Daten werden lokal in einer Datenbank gespeichert. Die Nutzer haben direkten Zugriff auf die Daten mit ihnen zugeordneten Zugriffsrechten. Dies ist ein Verfahren für große und komplexe Datenmengen an Einzelarbeitsplätzen.

Tabelle 15: Vor-und Nachteile einer dezentralen, datenbankbasierten Datenhaltung

\begin{tabular}{l|l}
\hline Vorteile & Nachteile \\
\hline+ einfacher und schneller Zugriff auf & - Spezialwissen über Datenbank- \\
alle Daten durch thematische oder & administration erforderlich \\
räumliche Suchfunktion & - höherer Aufwand für Installation, \\
+ komplexe Auswertungen möglich & Verwaltung und Pflege \\
+ Vermeidung von Daten- & - höhere Kosten durch Datenbank- \\
redundanzen & software \\
+ einfachere und bessere Möglichkei- & - Zugriff nur über entsprechende Pro- \\
ten der Datensicherung & gramme \\
\hline
\end{tabular}

\subsubsection{Rasterdatenmanagementsysteme}

Eine spezielle Form der zentralen Datenhaltung sind Rasterdatenmanagementsysteme (RDM). Rasterdatenmanagementsysteme dienen dem Management, der Verarbeitung und Bereitstellung großer Mengen dateibasierter Rasterdaten. Diese werden auf einem Rasterdaten-Speichersystem zentral gehalten und bei Bedarf nutzerspezifisch aufbereitet. Das Managementprogramm liegt auf einem sog. Applica- 
tion-Server. Die zugehörigen Metadaten werden in der Regel getrennt auf einem Datenbank-Server gehalten.

Optimierungsaspekte des RDM sind:

- Vermeidung asynchroner und manueller Verteilung der Daten, da diese sehr arbeitsintensiv ist und zu inkonsistenten Datenbeständen führt

- Standardisierung der Metadatenverwaltung, um eine intelligente Suche anhand von beispielsweise räumlichen und zeitlichen Kriterien zu erlauben (s. auch Kap. 8.3.3)

- Konformität zu OGC (Open GIS Consortium) und EU-Richtlinie INSPIRE (Infrastucture for Spatial Information in Europe)

- Zeitnahe und kontinuierliche Bedienung individueller Kundenwünsche ohne Mehrfachhaltung der Daten durch die Nutzung von Geoprocessing

- Einfache und effiziente Datenbereitstellung für vorhandene Fachsysteme, zum Beispiel mit unterschiedlichen Auflösungen, Koordinatensystemen und Komprimierungsstufen

\subsection{Hardwareausstattung von Datenservern}

Ein Server setzt sich, wie die meisten anderen PCs auch, im Wesentlichen aus den Hauptbestandteilen Prozessor(en), Arbeitsspeicher, Grafikeinheit und Festplatte(n) zusammen. Besonders an Prozessor(en), Arbeitsspeicher und Festplatte(n) werden gegenüber normalen Desktop-Systemen erhöhte Anforderungen gestellt.

Server-Prozessoren mit großen internen Speichern (Caches) sowie großen Registerbreiten (z. B. 64 Bit) müssen leistungsstark und langlebig sein.

Die Kapazität des Arbeitsspeichers ist bei Serversystemen größer als bei Desktop-Rechnern. Im Vordergrund steht auch hier die Zuverlässigkeit, weshalb spezielle, sich selbst überprüfende (Parity-Check) Speicherbausteine eingesetzt werden.

Die Festplatten des Servers sollten qualitativ hochwertig und langlebig sein sowie ausreichend Speicherplatz bieten. Für schnellen Datenzugriff und sichere Datenspeicherung sind besonders Raid-Systeme geeignet, die aus mehreren, zu einem virtuellen Laufwerk zusammengesetzten Festplatten bestehen. Ein wesentlicher Vorteil eines Raid-Systems ist die erhöhte Ausfallsicherheit. Jederzeit kann eine Festplatte ausgetauscht werden, ohne dass gespeicherte Daten verloren gehen oder das System ausfällt. 
Tabelle 16: Vorteile und Funktionalitäten eines Rasterdatenmanagementsystems

\begin{tabular}{|c|c|}
\hline Vorteile & Erläuterung der Funktionalität \\
\hline $\begin{array}{l}\text { Optimierung und Automatisierung } \\
\text { der Datenaufbereitung und -haltung }\end{array}$ & $\begin{array}{l}\text { - Weitestgehend redundanzfreie Datenhaltung } \\
\text { - Einfache und effiziente Datenaufbereitung }\end{array}$ \\
\hline Standardisiertes Datenmodell & $\begin{array}{l}\text { - ISO/OGC-konforme, standardisierte Bild- und Meta- } \\
\text { datenhaltung (ISO 19115/19139) } \\
\text { - Interaktion mit bestehenden Metainformationssystemen }\end{array}$ \\
\hline $\begin{array}{l}\text { Anwendungsgerechte } \\
\text { Datenbereitstellung und } \\
\text { Datennutzung }\end{array}$ & $\begin{array}{l}\text { - Zeitreihenanalysen } \\
\text { - Mosaikierung, Orthophotoberechnung und } \\
\text { Koordinatentransformation on the fly } \\
\text { - Nutzerspezifische Auflösungen } \\
\text { - Footprints } \\
\text { - Kundenspezifische Datendienste (zum Beispiel Update- } \\
\text { Service, WMS-Dienste) } \\
\text { - Protokollierung von Aufträgen und Zugriffen } \\
\text { - Einrichtung individueller Nutzerprofile } \\
\text { - Bedienung von Applikationen, die keine Webdienste } \\
\text { nutzen } \\
\text { - Integration in bestehende Webapplikationen }\end{array}$ \\
\hline \multicolumn{2}{|l|}{ Verarbeitung großer Datenmengen } \\
\hline $\begin{array}{l}\text { Schneller Datenzugriff und } \\
\text { hochperformante Datenbereit- } \\
\text { stellung bei geringer Netzbelastung }\end{array}$ & $\begin{array}{l}\text { - Von Dateimengen unabhängige Bereitstellung der Bild- } \\
\text { daten (zum Beispiel über waveletbasiertes Streaming von } \\
\text { ecw- und jpg2000-Dateien) } \\
\text { - Schnelle Visualisierung in Netzwerken }\end{array}$ \\
\hline Unterstützung diverser Bildformate & - Z. B. TIFF, MrSid, JPG2000, PDF, IMG, ECW, ... \\
\hline $\begin{array}{l}\text { Verringerung des } \\
\text { Speicherplatzbedarfs }\end{array}$ & $\begin{array}{l}\text { - Durch Bereitstellung von Diensten müssen keine } \\
\text { Zwischenprodukte vorgehalten werden; Datenverteilung } \\
\text { auf Anfrage (z. B. Orthophotoberechnung, Resampling } \\
\text { unter Eingabe der Bodenauflösung) } \\
\text { - Datenvisualisierung gemäß Darstellungsvorschriften (z. B. } \\
\text { Bandkombinationen, Bildstreckung, Schummerung von } \\
\text { Geländemodellen) }\end{array}$ \\
\hline $\begin{array}{l}\text { Flexible und hochperformante } \\
\text { Suchfunktionalitäten }\end{array}$ & $\begin{array}{l}\text { - Suchmaschinen für Geo- und Metadaten } \\
\text { - Vielfältige Suchfunktionalitäten, beispielsweise graphische } \\
\text { (Rechtecke, beliebiges Polygon) oder schlüsselwort- } \\
\text { bezogene Datensuche } \\
\text { - Einbindung von Diensten zur Suchvereinfachung }\end{array}$ \\
\hline $\begin{array}{l}\text { Reduktion des laufend anfallenden } \\
\text { Administrations- und } \\
\text { Sicherungsaufwandes }\end{array}$ & \\
\hline $\begin{array}{l}\text { Automatische Benachrichtigung bei } \\
\text { Datenaktualisierungen }\end{array}$ & \\
\hline $\begin{array}{l}\text { Optimierte Nutzerverwaltung / } \\
\text { Personalisierung von Rechten }\end{array}$ & $\begin{array}{l}\text { - Nutzer- und datenspezifische Rechte regeln den Zugriff } \\
\text { auf Bild- und Geodaten über Webdienste (WMS, WCS, } \\
\text { WFS) entsprechend unterschiedlicher Nutzerprofile }\end{array}$ \\
\hline
\end{tabular}




\subsection{Softwareausstattung eines Datenbankservers}

\subsubsection{Betriebssystem}

Als Betriebssystem kommen sowohl diverse UNIX-Systeme als auch Linux oder Windows infrage. Alle diese Systeme bieten verschiedene Vor- und Nachteile und die Entscheidung richtet sich normalerweise nach den Anforderungen an den Server, der eingesetzten Datenbank-Software und dem Wissensstand der Administratoren.

\subsubsection{Datenbank}

In der heutigen Zeit werden nahezu ausschließlich relationale Datenbanksysteme eingesetzt, deren Grundfunktionen über die Datenbanksprache SQL angesprochen werden. Die Hersteller von Datenbanken erweitern momentan ihre relationale Datenbanksoftware um objektorientierte Eigenschaften. Man spricht dann von objektrelationalen Datenbanken. Die existierenden Softwarelösungen besitzen unterschiedliche Vor- und Nachteile. Die Entscheidung, welche Datenbanksoftware eingesetzt wird, hängt daher auch hier stark vom Anforderungsprofil ab.

\subsubsection{Metadaten}

Metadaten sind „Daten über Daten“. Sie treffen Aussagen über die Eigenschaften und die Struktur von Datensätzen. Metadaten gestalten die Datenverwaltung effektiver, verhindern eine redundante Haltung der Daten und erleichtern deren Auffinden. Zudem sind diese Informationen wichtig für eine effiziente Nutzung der Daten, insbesondere bei einer Archivierung. Eine kontinuierliche Pflege der Metadaten ist erforderlich. Für die Erstellung von Metadaten existieren Softwarelösungen. Nachfolgend sind wichtige Bausteine eines Metadatensatzes aus dem Bereich Fernerkundung aufgelistet:

- Datenformat

- Komprimierungsformat

- Sensortyp

- Datenqualität

- Entstehungsdatum / Aktualität

- Produzent / Ansprechpartner

- Koordinatensystem

- Datenquelle / Entstehung der Daten

- Befliegungs- bzw. Orientierungsparameter 
Die EU-Richtlinie INSPIRE (s. http://www.inspire-geoportal.eu/) hat seit dem 15.07.2007 ein neues Kapitel im Bereich Bereitstellung von Meta- und Geodaten für die Öffentlichkeit aufgeschlagen. Sie verlangt von allen geodatenproduzierenden Institutionen auf lokaler, regionaler, bundes- und europaweiter Ebene zuerst die Bereitstellung von standardisierten Metadaten und in einem zweiten Schritt die Bereitstellung der Geodaten, z. B. als WMS-Dienst (Web Map Service).

Die in der INSPIRE Richtlinie geforderten Metadaten lehnen sich stark an die Spezifikation der Core-Metadaten der ISO-Norm 19115 an. Bei der Norm handelt es sich um eine Abfrage von ca. 400 Parametern zu einem einzelnen Datensatz. Bei den Core-Parametern handelt es sich um ca. 20 elementare Parameter. Über das Datenaustauschformat XML werden die standardisiert erfassten Daten an Metadatenkataloge der EU, der Länder, der Regionen oder privater Dienstleister übermittelt, zum Beispiel http://geodatenkatalog.hessen.de/. Anhand dieser Kataloge können Nutzer gezielt nach Metadaten oder aber nach Diensten, z. B. WMS oder WFS (Web Feature Service), für ihr Projektgebiet suchen.

\subsection{Datennetzwerke}

Der Zugriff auf Serverdaten ist abhängig von der Leitungskapazität, der Anzahl der Nutzer und der technischen Ausstattung des Datenservers. Man unterscheidet bei Datenleitungen zwischen Funk-, Laser- oder Kabelverbindungen. Kabelverbindungen sind aktuell am weitesten verbreitet, da sie relativ unempfindlich gegenüber Störungen sind. Eine Limitierung der Transfergeschwindigkeit im Netzwerk kann durch einen oder mehrere der oben beschriebenen Parameter verursacht werden. Daher ist eine optimale Konfiguration der unterschiedlichen Bausteine zueinander wichtig.

\subsection{Datensicherung}

Digitale Daten müssen gesichert werden. Dies geschieht durch temporäre Sicherungen (Backup) und langfristige Sicherungen (Archivierung).

Je nach Sicherungskonzept nutzt man unterschiedliche Formen des Backups (Full Backup, differenzielles Backup oder inkrementelles Backup). Unter Full Backup versteht man jeweils die komplette Sicherung eines Datenbestandes, zum Beispiel eines Laufwerkes. Bei einem differenziellen Backup werden alle Daten, die nach einem Full Backup erstellt oder verändert worden sind, auf ein Sicherungsmedium übertragen. Im Unterschied dazu werden beim inkrementellen Backup nur die Veränderungen seit der letzten inkrementellen Sicherung gespeichert.

Unabhängig von der Strategie muss die Aktualität der Sicherungen, die sorgfältige Verwahrung der Datenträger, die Überprüfung der Vollständigkeit des Backups und die Wiederherstellbarkeit der Daten gewährleistet sein. Die begrenzte Lebensdauer der eingesetzten Hard- und Software muss beachtet werden. 
Die Sicherung der Daten erfordert ein Konzept, kostet Zeit und Geld. Mit der Datenbeschaffung ist daher auch zu prüfen, welche Daten in welcher Form archiviert werden sollen. Es muss gewährleistet sein, dass die personellen und finanziellen Mittel für diese Aufgabe nachhaltig bereitstehen.

Für langfristig archivierte Daten kann der technische Fortschritt zu einer Gefahr werden. Mit der Einführung neuer Hard- oder Softwareausstattung ist es möglich, dass die vorhandenen Speichermedien oder -formate nicht mehr gelesen werden können. In der Übergangsphase kann es daher erforderlich werden, den gesamten Datenbestand in neue Formate zu konvertieren oder auf neue Datenträger zu übertragen.

Für eine Datensicherung kommen verschiedene Arten von Speichermedien (Magnetbänder, Festplatten, DVD, CD) in Betracht. Die unterschiedlichen Datenträger besitzen Vor- und Nachteile. Die Wahl des Speichermediums richtet sich nach dem Verwendungszweck der Datensicherung.

Für die vollautomatische Datensicherung werden häufig Magnetbänder (Tapes) und Bandroboter eingesetzt. Bandroboter sind Bandlaufgeräte, die eine variable Anzahl an Magnetbändern aufnehmen können und softwaregesteuert das Beschreiben der Tapes mit Backupdaten durchführen.

Die theoretisch erreichbare Lebensdauer von Speichermedien ist in der Praxis oft weniger ausschlaggebend als die rasche Veränderung der technischen Lösung der Datenspeicher (Veränderung von Formaten, Lesbarkeit alter Daten etc.).

Auf dem Markt existieren unterschiedlichste Softwareprodukte zur Datensicherung, teilweise schon im Betriebssystem integriert, die alle ihre Vor- oder Nachteile besitzen. Die Entscheidung, welche Produkte eingesetzt werden, hängt von der Anforderung an die Software sowie der vorhandenen Hardware und den Geldmitteln ab.

Die rasante technische Entwicklung sollte bei jeder Auswahl der Hard- und Software beachtet werden. 


\section{Hard- und Software für die Verarbeitung digitaler Luftbilder}

\subsection{Hardware}

Die Verarbeitung von digitalen Bilddaten bedeutet den Umgang mit großen Datenmengen. Um die Daten effizient bearbeiten zu können (speziell bei Stereoauswertungen), ist eine lokale Speicherung oder eine sehr schnelle Netzverbindung notwendig.

Für photogrammetrische Weiterbearbeitungen (Triangulation, DOM- und Orthophotoherstellung) genügt ein leistungsfähiger Standard-PC mit ausreichendem Arbeitsspeicher und einer hohen Festplattenkapazität. Gleiches gilt für die 2D-Auswertung von Orthophotos.

Für die stereoskopische Auswertung ist spezielle Hardware erforderlich. Die Grafikkarte muss für Stereodarstellung geeignet, mit einem ausreichend großen Arbeitsspeicher ausgestattet und auf das gesamte Auswertungssystem abgestimmt sein. Die überlappenden orientierten Luftbilder müssen so dargestellt werden, dass mit dem linken Auge das linke Bild und mit dem rechten Auge das rechte Bild sichtbar ist, um den 3D-Eindruck zu erhalten. Aktuell gibt es zwei verschiedene Techniken, die sich in Ergonomie, Bilddarstellung und in den Anschaffungskosten unterscheiden.

Beim aktiven System werden beide Luftbilder eines Stereomodells abwechselnd mit einer Frequenz von $60 \mathrm{~Hz}$ auf einem LCD-Bildschirm dargestellt. Der Bildschirm muss eine Bildwiederholungsrate von $120 \mathrm{~Hz}$ besitzen. Eine aktive Shutterbrille wird von der Grafikkarte gesteuert und mittels Flüssigkristallen in der gleichen Frequenz durchsichtig und lichtundurchsichtig geschaltet. Hierdurch entsteht der Eindruck, beide Bilder gleichzeitig und hierdurch stereo zu sehen.

Beim passiven System werden die beiden Bilder des Stereomodells auf zwei LCD-Monitoren dargestellt. Über einen halbdurchlässigen Polarisationsspiegel und durch eine passive Polarisationsbrille wird gleichzeitig jedes Bild auf ein Auge gelenkt. Der Vorteil von diesen Systemen gegenüber dem vorgenannten ist eine bessere Ergonomie.

In der Praxis hat sich für die stereoskopische Bearbeitung ein zusätzlicher Bildschirm neben dem 3D-System bewährt. Auf diesem werden die Sekundärdaten geführt.

Für eine 3D-Digitalisierung können die normale Systemmaus oder spezielle 3D-Eingabegeräte verwendet werden. Diese verfügen über Drehräder für die Steuerung der Messmarke. Außerdem besitzen sie programmierbare Knöpfe, die mit Funktionen belegt werden können. Dadurch muss wenig zwischen dem 3D-Erfas- 
sungsgerät und der Tastatur gewechselt werden. Bei der Systemmaus wird die Höheneingabe über das Scrollrad gesteuert.

\subsection{Software}

Für die photogrammetrische Bearbeitung digitaler und digitalisierter Luftbilder stehen verschiedene Softwarepakete zur Verfügung. Hiermit können Luftbilder von Matrixkameras und digitalisierte analoge Luftbilder umfassend bearbeitet werden (vgl. Kap. 2.2 und 3). Mit Einschränkungen gilt dies auch für Luftbilder von Zeilenkameras, für deren Orientierung spezielle Programme erforderlich sind.

Für die Datenerfassung in Stereobildern wird Spezialsoftware von verschiedenen Herstellern angeboten. Eine effiziente Datenerfassung und Kartierung erfordert eine gute Schnittstelle bzw. Einbettung des 3D-Erfassungsmoduls in eine Kartier- bzw. GIS-Umgebung. Bereits vorhandene Vektordaten sollten eingeblendet werden können. Um die hohe radiometrische Auflösung der digitalen Luftbilder nutzen zu können, sollten Werkzeuge für die Bildanpassung (Histogrammbearbeitung, dynamische Anpassung, schnelle Änderung von Helligkeit und Kontrast) vorhanden sein.

Die dreidimensionale Erfassung bietet zusätzliche Visualisierungsmöglichkeiten für planerische und repräsentative Zwecke. Durch eine perspektivische Darstellung der Objekte kann ein leicht verständliches und informatives 3D-Bild erzeugt werden. 


\section{Fazit}

Der vorliegende Leitfaden gibt Anwendern wesentliche, aktuelle Informationen zur Aufnahme und Verarbeitung digitaler Luftbilder. Die einzelnen Kapitel setzen folgende Schwerpunkte:

\subsection{Digitale Luftbildkameras (Kapitel 2)}

Digitale Luftbildkameras unterscheiden sich erheblich von analogen Kameras. Es gibt Matrixkameras verschiedener Sensorgrößen und Zeilenkameras. Meist werden digitale Luftbilder mit den vier Farbkanälen Rot, Grün, Blau und Nahes Infrarot aufgezeichnet. Der Nutzer muss entsprechend seiner Fragestellung, der Größe der zu erkennenden Objekte, der Art der Auswertung und der vorhandenen Hard- und Software über die erforderliche Bodenauflösung, radiometrische Auflösung und Kanalkombination entscheiden.

\subsection{Digitalisierung analoger Luftbilder (Kapitel 3)}

Es gibt umfangreiche Archive analoger Luftbilder aus den letzten 100 Jahren. Um moderne, digitale Auswertetechniken und -methoden bei diesen Bildern einsetzen zu können, muss das Filmmaterial digitalisiert werden. Der Einsatz von Scantechnik und -verfahren erfolgt in Abhängigkeit von den Genauigkeitsanforderungen. Für Interpretationszwecke ist die Präzision des Scanners weniger bedeutend, für photogrammetrische Messungen ist sie entscheidend. Bei der Digitalisierung können Bildverbesserungen durchgeführt werden.

\subsection{Planung, Vergabe und Durchführung digitaler Bildflüge (Kapitel 4)}

Die Planung eines digitalen Bildfluges erfordert eine Ausrichtung der technischen und äußeren Rahmenbedingen auf den jeweiligen Befliegungszweck. Zusätzlich zu den Anforderungen, die auch bei analogen Bildflügen zu beachten waren, sollte hierbei ein Fokus auf der Lieferung möglichst homogener Bilddaten sowie einer möglichst transparenten Nachprozessierung liegen. Beim gegenwärtigen Stand der Technik können nicht alle Wünsche der Nutzer nach Transparenz erfüllt werden.

Der Lieferumfang, sowie die Liefer- und Lizenzbedingungen müssen vor Auftragsvergabe exakt definiert und vertraglich vereinbart werden. 


\subsection{Bildorientierung (Kapitel 5)}

Die Stereoauswertung digitaler Luftbilder sowie die Erstellung von Orthophotos und Höhenmodellen erfordern Bildorientierungen. Aufgrund der bei digitalen Bildflügen gewonnenen externen Orientierungsparameter können diese heute gegenüber den analogen Bildorientierungen deutlich schneller durchgeführt werden. Bei größeren Ansprüchen an die Lage- und Höhengenauigkeit von Messdaten sind Aerotriangulationen unter Einbeziehung von Passpunkten erforderlich.

\subsection{Orthophotoerstellung (Kapitel 6)}

Orthophotos werden heute mit vergleichsweise geringem zusätzlichen Aufwand aus orientierten digitalen Luftbildern unter Verwendung von digitalen Höhenmodellen berechnet. Hierfür werden Oberflächen- oder Geländemodelle genutzt. Diese können unter anderem von den Vermessungsämtern bezogen werden. Oberflächenmodelle lassen sich auch aus digitalen Stereomodellen automatisiert berechnen. Die Qualität der Orthophotos ist maßgeblich von der Qualität des Höhenmodells abhängig.

\subsection{Auswertung digitaler Luftbilder (Kapitel 7)}

Digitale Luftbilder können visuell oder teilautomatisiert ausgewertet werden. Schnelle Bildwechsel, Farbanpassungen, digitale Bildverarbeitung und die Integration in GIS-Arbeitsumgebungen erlauben ein effizientes Arbeiten. Allerdings fehlen noch Standards für Bildverbesserungen. Automatisierte Auswertungen stoßen in der Praxis an ihre Grenzen. Der Schwerpunkt thematischer Auswertungen liegt immer noch auf der visuellen Interpretation. Das Kapitel enthält Anwendungsbeispiele.

\subsection{Datenmanagement (Kapitel 8)}

Ein effizientes Management von Daten ist eine wesentliche Voraussetzung für die Arbeit mit digitalen Bilddaten. Schnelle und permanente Zugriffe auf zum Teil große Datenmengen sind erforderlich. Datenredundanzen sollten vermieden werden. Standardisierte Metadaten, eine verlustfreie Datenhaltung und die sorgfältige Archivierung sind Grundvoraussetzungen für eine langfristige Nutzbarkeit der Daten. 
10.8 Hard- und Software für die Verarbeitung digitaler Luftbilder (Kapitel 9)

Die bei der Verarbeitung digitaler Luftbilder anfallenden großen Datenmengen sind bei der Konfiguration von Rechnerumgebungen zu berücksichtigen. Stereoskopisch-photogrammetrische Auswertungen erfordern spezielle und besonders leistungsfähige Grafikkarten und Bildschirme. Die Bearbeitung und Auswertung digitaler Luftbilder ist nur mit spezieller Software möglich. 



\section{Literatur}

Albertz, J.; Rodehorst, V.; Gwinner, K.; HOHMUth, F.; RoAtsch, T.; SCHOLTEN, F. u. WiEDEMANN, A. (2002): FIGARO - Fernerkundung im Gigabit-Net - Automatische Rekonstruktion von Oberflächen. Abschlussbericht zu dem vom Deutschen Forschungsnetz geförderten Forschungsprojekt (03/99 bis 10/01), $55 \mathrm{~S}$.

AlBERTZ, J. (2007): Einführung in die Fernerkundung - Grundlagen der Interpretation von Luft- und Satellitenbildern. Darmstadt, 264 S.

ARBEITSGRUPPE FORSTLICHER LUFTBILDINTERPRETEN (AFL) (1998): LuftbildInterpretationsschlüssel, Bestimmung der natürlichen Altersklasse von Waldbeständen im Color-Infrarot-Luftbild. LÖBF-Mitteilungen Nr. 1/1998, 45-50

ARBEITSGRUPPE FORSTLICHER LUFTBILDINTERPRETEN (AFL) (1999): LuftbildInterpretationsschlüssel II, Bestimmung der natürlichen Altersklassen und der Baumarten von Waldbeständen im CIR-Luftbild. LÖBF-Mitteilungen Nr. 4/1999, 51-56

ARBEITSGRUPPE FORSTLICHER LUFTBILDINTERPRETEN (AFL) (2003): LuftbildInterpretation - Bestimmungsschlüssel für die Beschreibung von strukturreichen Waldbeständen im Color-Infrarot-Luftbild. Landesforstpräsidium Freistaat Sachsen, Schriftenreihe Nr. 26, 48 S.

Congalton, R.G. (1983): A quantitative method to test for consistency and correctness in photo interpretation. Photogrammetric Engineering and Remote Sensing 49(1), 69-74

Congalton, R.G.; Green, K. u. Russel, G. (1999): Assessing the accuracy of remotely sensed data: Principles and practices. Boca Raton, $137 \mathrm{~S}$.

Deutsches Institut FÜr Normung E.V. (2005): DIN 18740-2 Photogrammetrische Produkte - Teil 2: Anforderungen an das gescannte Luftbild. Berlin, $17 \mathrm{~S}$.

DeUtsches Institut FÜr Normung E.V. (2011): DIN 18716-4 Photogrammetrie und Fernerkundung - Begriffe. Berlin, $35 \mathrm{~S}$.

European Commission (Hrsg.) (2000): Remote Sensing Applications for Forest Health Status Assessment. Luxemburg, 216 S.

Geosystems (2001): Luftbilder in der digitalen Photogrammetrie. White Paper GEOSYSTEMS GmbH, $20 \mathrm{~S}$. 
GRIEßMANN, P. (2007): Erfassung von Waldstruktur- und Waldzustandsparametern aus Digitalen Luftbilddaten der sachsenweiten Befliegung 2005. Diplomarbeit, TU Dresden, Institut für Kartographie, $80 \mathrm{~S}$.

Gross, C.P. u. ADLER, P. (1996): Reliability of area mapping by delineation in aerial photographs. Proceedings Spatial Accuracy assessment in natural resources and environmental sciences, Fort Collins, USDA Forest Service general technical report RM-GTR-277, 267-271

HilDEBRANDT, G. (1996): Fernerkundung und Luftbildmessung für Forstwirtschaft, Vegetationskartierung und Landschaftsökologie. Heidelberg, 676 S.

LU, D. u. WENG, Q. (2007): A survey of image classification methods and techniques for improving classification performance. Int. J. of Remote Sensing, 28, $823-870$

SANDAU, R. (Hrsg.) (2005): Digitale Luftbildkamera - Einführung und Grundlagen. Heidelberg, $342 \mathrm{~S}$.

VEREIN DEUTSCHER INGENIEURE (VDI) (1993): VDI 3793 Messen von Vegetationsschäden am natürlichen Standort, Blatt 2 Interpretationsschlüssel für die Auswertung von CIR-Luftbildern zur Kronenzustandserfassung von Nadelund Laubgehölzen; Fichte, Buche, Eiche und Kiefer. Düsseldorf, 57 S. 


\section{Abkürzungen}

2D

Zweidimensional

3D

Dreidimensional

AFL

Arbeitsgruppe Forstlicher Luftbildinterpreten

ALS

Airborne Laser Scanning

CCD

Charge-coupled Device

CIR

Color-Infrarot

DGM

Digitales Geländemodell

DGPS

Differential Global Positioning System

DHM

Digitales Höhenmodell

DIN

Deutsche Institut für Normung e.V.

DOM

Digitales Oberflächenmodell

DOP

Digitales Orthophoto

DSM

Digital Surface Model

DTM

Digital Terrain Model

FFH

Fauna Flora Habitat

GIS

Geographisches Informationssystem

GNSS

Global Navigation Satellite System

GPS

Global Positioning System

GSD

Ground Sample Distance

IMU

Inertial Measurement Unit

INS

Inertial Navigation System

INSPIRE

Infrastrukture for Spatial Information in Europe

ISO

International Organisation for Standardisation

LCD

Liquid Crystal Display

LIDAR

Light Detection and Ranging

NDVI

Normalized Difference Vegetation Index

NIR

Nahes Infrarot

OGC

Open Geospatial Consortium

PAN

Panchromatisch

PCA

Principal Component Analysis

$\mathrm{RDM}$

Rasterdatenmanagementsystem

RGB

Rot Grün Blau 
SW

WCS

WFS

WMS
Schwarzweiß

Web Coverage Service

Web Feature Service

Web Map Service 


\section{Glossar}

Aerotriangulation: Verfahren, bei dem die Einzelbilder einer Befliegung durch Verknüpfungs- und => Passpunkte zu einem Gesamtmodell zusammengefügt werden. Dieses wird für die $=>$ Bildorientierung und die Berechnung der äußeren Orientierung der Luftbilder verwendet.

ALS: Abkürzung für „Airborne Laser Scanning“. Hierbei handelt es sich um ein laserbasiertes Fernerkundungsverfahren zur hochgenauen Erfassung von Geländeund Vegetationsoberflächen. Aus so gewonnenen Daten werden hochgenaue $=>$ Digitale Gelände- und Oberflächenmodelle abgeleitet.

Analoges Luftbild: Durch Verfahren der analogen Photographie unter Verwendung von Filmmaterialien hergestelltes Schwarzweiß-, Normalfarb- oder $=>$ Farbinfrarot-Luftbild (s. auch => Digitales Luftbild).

Bildkanal: Teil einer digitalen Luftbild- oder Satellitenaufnahme, in dem Daten eines $=>$ Farbkanals oder daraus abgeleitete Produkte gespeichert sind.

Bildorientierung: Räumliche Einordnung von Bildinformationen in ein vorgegebenes $=>$ Referenz- bzw. Koordinatensystem durch photogrammetrische Verfahren.

Bit: Bezeichnung für eine Binärziffer, die die Werte 0 oder 1 annehmen kann. Kleinste Einheit in der Computertechnik zur Speicherung von Informationen.

Bodenauflösung: Die Bodenauflösung eines digitalen Luft- oder Satellitenbildes, auch ground sample distance (GSD) genannt, gibt die Größe eines => Pixels in der realen Welt an.

Byte: Datenspeicherungseinheit, bestehend aus 8 Bit (=> Bit), mit einem Wertebereich von 0-255.

CCD-Sensor: Lichtempfindliches elektronisches Bauteil (charge-coupled device) zur digitalen Erfassung von Helligkeitswerten; wesentlicher Bestandteil digitaler Bildaufnahmesysteme.

CIR-Luftbild: siehe $=>$ Farbinfrarot-Luftbild

Digitales Geländemodell (DGM): Numerisches Höhenmodell der Erdoberfläche ohne Berücksichtigung darauf befindlicher natürlicher und künstlicher Objekte (z. B. Vegetation oder Bebauung). Die englische Bezeichnung ist Digital Terrain Model (DTM).

Digitales Luftbild: Mit einer digitalen Kamera aufgenommenes Luftbild, dessen Bildinformationen pixelweise in Form digitaler Zahlenwerte gespeichert werden. Digitale Luftbilder bestehen meist aus mehreren $=>$ Bildkanälen (z. B. Rot, Grün, Blau, Nahes Infrarot) und besitzen $=>$ radiometrische Auflösungen von 8 oder 16 Bit (=> Bit) pro => Bildkanal (s. a. => Analoges Luftbild). 
Digitales Oberflächenmodell (DOM): Numerisches Höhenmodell der Erdoberfläche unter Einbeziehung darauf befindlicher natürlicher oder künstlicher Objekte (z. B. Vegetation oder Bebauung). Die englische Bezeichnung ist Digital Surface Model (DSM).

Digitalisierung: Umwandlung analoger Informationen in digitale Daten.

Drehwinkel: Die Drehwinkel $\omega, \varphi$ und $x$ definieren die Lage der Bildebene einzelner Luftbilder im Raum im Moment ihrer Aufnahme. Der Winkel $\omega$ (Omega) beschreibt die Neigung quer zur Flugrichtung, $\varphi$ (Phi) die Längsneigung in Flugrichtung und $x$ (Kappa) die Flugrichtung. Diese Informationen werden durch eine Inertial Measurement Unit (IMU) erfasst und für die photogrammetrische $=>$ Bildorientierung verwendet.

Farbinfrarot-Luftbild: Farbinfrarot-Luftbilder, auch CIR-Luftbilder genannt, entstehen unter Einbeziehung von Strahlung aus dem Wellenlängenbereich des Nahen Infrarot (700-1400 nm). Digital aufgenommene Farbinfrarot-Luftbilder sind dreikanalige Bilder, bestehend aus den drei $=>$ Bildkanälen Nahes Infrarot (NIR), Rot $(\mathrm{R})$ und Grün $(\mathrm{G})$.

Farbkanal: Spektralbereich, der von einem Fernerkundungssensor aufgenommen werden kann. Multispektralsensoren verfügen über mehrere Farbkanäle.

Farbtiefe: Anzahl darstellbarer bzw. unterscheidbarer Farben bei digitalen Bildern, häufig auch als $=>$ radiometrische Auflösung bezeichnet. Die Farbtiefe wird kanalweise über die Anzahl der für die Speicherung der jeweiligen => Grauwerte zur Verfügung stehenden $=>$ Bits definiert. Üblich sind bei digitalen Luftbildern 8 oder 16 Bit pro => Bildkanal, was 256 bzw. 65.536 Abstufungen entspricht.

Geo-Informationssystem (GIS): System aus Hardware, Software, Daten und Anwendungen, mit dem raumbezogene Informationen erfasst, gespeichert, analysiert und dargestellt werden können.

Geometrische Auflösung: Beschreibt die räumliche Auflösung eines Sensors. Sie beeinflusst zusammen mit der radiometrischen Auflösung die Erkennbarkeit von Objekten und Strukturen.

Geometrische Genauigkeit: Beschreibt die Genauigkeit / Konsistenz der geometrischen Auflösung

GNSS: Abkürzung für Global Navigation Satellite System als Oberbegriff für weltweite, satellitengestützte Systeme zur Positionsbestimmung auf oder über der Erdoberfläche, wie bspw. => GPS.

GPS: Abkürzung für Global Positioning System, ein amerikanisches $=>$ GNSS. GPS ist öffentlich nutzbar. Einfache Empfangsgeräte erreichen eine Lagegenauigkeit von 5-10 m. Mit höherem technischen Aufwand lassen sich diese Werte bis auf wenige Zentimeter verbessern. Bei Luftbildbefliegungen wird die räumliche Lage der Flugzeuge mit hochgenauen differentiellen Empfängern (Differential Global Positioning System (DGPS)) erfasst. 
Grauwert: Helligkeitswert eines => Pixels bei $=>$ Rasterdaten. Die Anzahl der nutzbaren Grauwerte hängt dabei von der verfügbaren Anzahl an $=>$ Bits ab. 8 Bit -Bilder erlauben z. B. die Darstellung von 256 Grauwerten. Farbbilder entstehen durch die Verknüpfung von Grauwerten mit Farbtabellen.

Histogramm: Häufigkeitsverteilung von => Grauwerten in graphischer Form.

Höhenmodelle: siehe $=>$ Digitales Geländemodell und $=>$ Digitales Oberflächenmodell

INS: Abkürzung für Inertial Navigation System. INS-Systeme werden zusammen mit => GNSS-Geräten bei Luftbildbefliegungen für die Positions- und Geschwindigkeitsbestimmung eingesetzt. Sie sind eine wesentliche Voraussetzung für die Ermittlung qualitativ hochwertiger Orientierungsparameter $(=>$ Drehwinkel $\omega, \varphi$ und $x$ ).

Kalibrierungsprotokoll: Dokument, mit dem die Kalibrierung von Luftbildkameras bescheinigt wird und das alle wesentlichen, für eine Luftbildorientierung erforderlichen Kamera- und Objektivdaten enthält.

Klassifizierung: Auswertungsmethode der digitalen Bildverarbeitung, durch die bei Rasterbildern Flächen ähnlicher Eigenschaften (Farbe, Textur) ermittelt werden. Dabei kommen objektorientierte oder pixelbasierte Verfahren zum Einsatz.

Matrixkamera: Digitale Luftbildkamera, bei der die Photodioden der $=>$ CCDSensoren flächig in einer Matrix angeordnet sind und die rechteckige, zentralperspektivische Aufnahmen liefert.

Metadaten: Informationen über Daten. Sie beinhalten Aussagen über die Eigenschaften sowie die Struktur von Datensätzen und werden beim Management meist umfangreicherer Datensammlungen verwendet.

Nadirpunkt: Bodenpunkt vertikal unter der Kamera oder dem Sensor eines Fernerkundungssystems. Entsprechend werden Aufnahmen mit genau lotrechter Ausrichtung als Nadiraufnahmen bezeichnet.

Orientiertes Luftbild: Digitales Luftbild, mit dem alle wesentlichen Orientierungsinformationen gespeichert sind ( $\mathrm{x}-, \mathrm{y}-$ und $\mathrm{z}$-Werte des Projektionszentrums, Drehwinkel $\omega, \varphi$ und $x$ sowie Kamerakalibrierungsdaten) und das hierdurch direkt ohne weitere Aufbereitungsschritte für digitale Stereoauswertungen verwendet werden kann.

Orthophoto: Aus Luftbildern oder Satellitenaufnahmen erzeugte, verzerrungsfreie und maßstabsgetreue Abbildung der Erdoberfläche. Digitale Orthophotos entstehen in einem photogrammetrischen Prozess unter Verwendung $=>$ digitaler Gelände- oder Oberflächenmodelle.

Panchromatischer Bildkanal (PAN): => Farbkanal eines optischen, digitalen Sensors (Luftbildkamera, Satellitensensor), der eine über den gesamten Bereich des sichtbaren Lichtes reichende Empfindlichkeit besitzt. Die Abstufung der Grauwerte panchromatischer Daten ist vergleichbar mit typischen Schwarzweiß-Bildern. 
Pansharpening: Nachprozessierung digitaler Luft- und Satellitenbildaufnahmen durch die Fusion von $=>$ panchromatischen Bilddaten hoher Auflösung mit geringer aufgelösten Spektraldaten. Dies dient der Verbesserung der geometrischen Auflösung spektraler Daten.

Passpunkte: Geländepunkte mit bekannten Koordinatenwerten, die für die Orientierung von Luft- oder Satellitenbildern verwendet werden.

Pixel: Zelle eines Rasterdatensatzes, definiert durch eine eindeutige Position in der Bildmatrix und durch einen zugeordneten $=>$ Grauwert. Die Pixelgröße im Bezug zum dargestellten Gelände entspricht der $=>$ Bodenauflösung und bestimmt weitgehend die Verwendbarkeit eines Bildes für bestimmte Auswertungszwecke.

Radiometrische Auflösung: Fähigkeit eines Sensors, Strahlungsunterschiede messtechnisch zu erfassen (DEUTSCHES INSTITUT FÜR NORMUNG 2011). Häufig wird dieser Begriff bei der digitalen Bildverarbeitung auch synonym für => Farbtiefe verwendet.

Rahmenmarken: Markierungen am Rand $=>$ analoger Luftbildaufnahmen zur inneren Orientierung der Bilder.

Rasterdaten: Digitale Daten, die als Matrix in Zeilen und Spalten angeordnet vorliegen. Die einzelnen Rasterzellen werden $=>$ Pixel genannt.

Referenzsystem: Koordinatensystem, das als Bezugssystem für die Orientierung von Luftbildern und Satellitenaufnahmen verwendet wird.

RGB: Additives Farbmodell, bestehend aus den Grundfarben Rot, Grün und Blau. Das RGB-Modell wird für die Bildschirmdarstellung benutzt, meist mit einer Farbtiefe von 8 Bit (=> Bit) pro Grundfarbe. Hierdurch stehen insgesamt über 16 Millionen Farben zur Verfügung.

Scanauflösung: Geometrische Auflösung der beim Abscannen analoger Vorlagen entstehenden Rasterbilder. Angabe in $\mu \mathrm{m}$ (tausendstel Millimeter) oder dpi (dots per inch).

Scanner: Datenerfassungsgerät zur Digitalisierung analoger Vorlagen, z. B. Luftbilder. Mit Hilfe eines Sensors werden die Vorlagen in digitale Werte überführt und anschließend in einem Rasterformat abgespeichert.

Segmentierung: Methode der Bildbearbeitung, bei der benachbarte $=>$ Pixel mit ähnlichen Eigenschaften nach vorgegebenen Kriterien zu Bildausschnitten zusammengefasst werden, bspw. als Vorstufe zur $=>$ Klassifizierung digitaler Bilddaten.

Stereoluftbild: Paar benachbarter Luftbilder, die in ihrem Überlappungsbereich eine stereoskopische Betrachtung der aufgenommenen Objekte zulassen.

Trainingsgebiete: Verwendung bei der $=>$ Klassifizierung von Luftbild- oder Satellitenaufnahmen. Hierbei handelt es sich um Gebiete, deren terrestrische Eigenschaften bekannt sind und die als Referenz bei der rechnergestützten Bildanalyse dienen. 
Zeilenkamera: Digitale Luftbildkamera, bei der die Photodioden ihrer $=>$ CCDSensoren zeilenförmig angeordnet sind. Hierdurch entsteht bei der Aufnahme ein beliebig langer, konstant breiter Bildstreifen. 



\section{Autoren}

\section{Jörg Ackermann}

Nordwestdeutsche Forstliche

Versuchsanstalt

Grätzelstraße 2

D-37079 Göttingen

joerg.ackermann@nw-fva.de

\section{Christoph Bauerhans1}

Bundesforschungs- und

Ausbildungszentrum für Wald

Seckendorff-Gudent Weg 8

A-1131 Wien

christoph.bauerhansl@bfw.gv.at

\section{Friedrich Engels}

Forschungsanstalt für Waldökologie und Forstwirtschaft Rheinland-Pfalz

Schloss

D-67705 Trippstadt

friedrich.engels@wald-rlp.de

\section{Christian Ginzler}

Eidgenössische Forschungsanstalt für Wald, Schnee und Landschaft

Zürcherstraße 111

CH-8903 Birmensdorf

christian.ginzler@wsl.ch

\section{Dr. Petra Adler}

Forstliche Versuchs- und

Forschungsanstalt Baden-Württemberg

Wonnhalde 4

D-79100 Freiburg

petra.adler@forst.bwl.de

\section{Uwe Brockamp}

ö:konzept GmbH

Heinrich-von-Stephanstraße 8b

D-79100 Freiburg

brockamp@oekonzept-freiburg.de

\section{Frank Franken}

Hessen-Forst, Servicezentrum für

Forsteinrichtung und Naturschutz

Europastraße 10-12

D-35394 Gießen

frank.franken@forst.hessen.de

\section{Dr. Claus-Peter Gross}

Universität Freiburg

Abteilung Fernerkundung und LIS

Tennenbacherstraße 4

D-79085 Freiburg

claus-peter.gross@felis.uni-freiburg.de 
Karina Hoffmann

Staatsbetrieb Sachsenforst

Bonnewitzer Straße 34

D-01796 Pirna OT Graupa

karina.hoffmann@smul.sachsen.de

\section{Prof. Dr. Hartmut Kenneweg}

TU Berlin

Institut für Landschaftsarchitektur und Umweltplanung

Sekr. EB 05, Straße d. 17. Juni 145

D-10623 Berlin

kenneweg@ile.tu-berlin.de

\section{Dr. Klaus Martin}

Sachverständigenbüro für

Luftbildauswertung und Umweltfragen

Kohlsteiner Straße 5

D-81243 München

klaus.martin.slu@t-online.de

\section{Rudolf Seitz}

Bayerische Landesanstalt für Wald und Forstwirtschaft

Hans-Carl-von-Carlowitz-Platz 1

D-85354 Freising

rudolf.seitz@lwf.bayern.de

\section{Kai Jütte}

Landesforst Mecklenburg-

Vorpommern

Zeppelinstr. 3

D-19061 Schwerin

kai.juette@lfoa-mv.de

\section{Dr. Tatjana Koukal}

Universität für Bodenkultur Wien

Institut für Vermessung,

Fernerkundung und Landinformation

Peter-Jordan-Straße 82

A-1190 Wien

tatjana.koukal@boku.ac.at

\section{Bruno Regner}

Bundesforschungs- und

Ausbildungszentrum für Wald

Seckendorff-Gudent Weg 8

A-1131 Wien

bruno.regner@bfw.gv.at

\section{Armin Troycke}

Bayerische Landesanstalt für Wald und Forstwirtschaft

Hans-Carl-von-Carlowitz-Platz 1

D-85354 Freising

armin.troycke@lwf.bayern.de 


\section{Anhang}

Muster einer Bildflugdokumentation und eines Datenübergabeprotokolls einer digitalen Luftbildbefliegung

a. Auftraggeber/ Auftragnehmer

b. Projektbezeichnung

c. Technischer Bericht mit Angaben zum Flug:

- Bildstreifen

- Bildnummern

- Flugtag

- Aufnahmezeit

- Flughöhe über Grund

- horizontale Sichtweite

- Bewölkung

- Bodenauflösung

- Längs-/Querüberdeckung

- verwendete Kamera

- Brennweite

- Blende

- Belichtungszeit

- verwendete Filter

- Erläuterungen von Besonderheiten und Abweichungen

- Bestätigung der Vollständigkeit des durchgeführten Flugs

d. Kalibrierungszertifikat der verwendeten Kamera inkl. der Spektralbereiche der Farbkanäle

e. Bildmittenübersicht/ Bildmittenkoordinaten

f. Art der gelieferten Bildprodukte mit der jeweiligen Bildkanalbelegung und gegebenenfalls Angabe vorgenommener zusätzlicher Prozessierungen

g. Dateiformat der Bilddaten

h. Datenträger 
i. Bezeichnung der Bilddateien, z. B. mit:

$\begin{array}{ll}\text { Ziffer } 1 \text { bis } 2 & \text { Jahr der Befliegung (z. B. 12 für 2012) } \\ \text { Ziffer } 3 & \text { Trennzeichen } \\ \text { Ziffer } 4 \text { bis } 7 & \text { Gebietsbezeichnung } \\ \text { Ziffer } 8 & \text { Trennzeichen } \\ \text { Ziffer } 9 \text { bis } 10 & \text { Streifennummer } \\ \text { Ziffer } 11 & \text { Trennzeichen } \\ \text { Ziffer } 12 \text { bis } 15 & \text { Bildnummer (fortlaufend im Flugstreifen) } \\ \text { Ziffer } 16 & \text { Trennzeichen } \\ \text { Ziffer } 17 \text { bis } 20 & \text { Kurzbezeichnung für Bildfarbformat (RGBI, } \\ & \text { RGB/CIR/PAN) } \\ \text { Ziffer } 21 & \text { Trennzeichen } \\ \text { Ziffer } 22 \text { bis } 23 & \text { Radiometrische Auflösung (8 Bit/16 Bit) }\end{array}$

Beispiel für eine Bilddateibezeichnung: 10_C306_01_0003_RGBI_16

j. Dokumentation der äußeren Orientierungsparameter:

- Art der Bestimmung der Orientierungsparameter (direkte oder indirekte Georeferenzierung)

- verwendetes Referenzsystem

- Maßeinheit der Drehwinkel

- Reihenfolge und Benennung der Spalten der Übergabedatei (z. B.: Streifennummer / Bildnummer / Aufnahmezeit / Rechtswert / Hochwert / Flughöhe ü. NN / Drehwinkel $\omega, \varphi$, und $x$ )

k. Beschreibung der Urheber- und Nutzungsrechte 


\section{Beiträge aus der Nordwestdeutschen Forstlichen Versuchsanstalt}

Band

1 (2007) Clusterstudie Forst und Holz Niedersachsen. Burkhard Rüther, Jan Hansen, Agatha Ludwig, Hermann Spellmann, Jürgen Nagel, Bernhard Möhring, Matthias Dieter. 92 S.

2 (2008) Die Waldkiefer - Fachtagung zum Baum des Jahres 2007. Nordwestdeutsche Forstliche Versuchsanstalt (Hrsg.). 98 S.

3 (2008) Ergebnisse angewandter Forschung zur Buche. Nordwestdeutsche Forstliche Versuchsanstalt (Hrsg.). 343 S.

4 (2008) Ergebnisse des westdeutschen IUFRO-Küstentannen-Provenienzversuches im Alter 27. Hans-Martin Rau, Armin König, Wolfhard Ruetz, Hendrik Rumpf, Egbert Schönfelder. $62 \mathrm{~S}$.

5 (2010) Fichtenherkunftsversuch von 1962 und IUFRO-Fichtenherkunftsversuch von 1972. Ergebnisse von mehr als 30jähriger Beobachtung in Deutschland. Mirko Liesebach, Hans-Martin Rau, Armin O. König. 467 S.

6 (2011) Kohlenstoffstudie Forst und Holz Niedersachsen. René Wördehoff, Hermann Spellmann, Jan Evers, Jürgen Nagel. 92 S.

7 (2012) Das digitale Luftbild. Ein Praxisleitfaden für Anwender im Forst- und Umweltbereich. Arbeitsgruppe Forstlicher Luftbildinterpreten (Hrsg.). $84 \mathrm{~S}$.

Alle Bände der „Beiträge aus der NW-FVA“ sind auch als freie Onlineversion über die Homepage der NW-FVA (www.nw-fva.de), des Verlages sowie über den OPAC der Niedersächsischen Staats- und Universitätsbibliothek

(http://www.sub.uni-goettingen.de) erreichbar und dürfen gelesen, heruntergeladen sowie als Privatkopie ausgedruckt werden. Es gelten die Lizenzbestimmungen der Onlineversion. Es ist nicht gestattet, Kopien oder gedruckte Fassungen der freien Onlineversion zu veräußern. 
